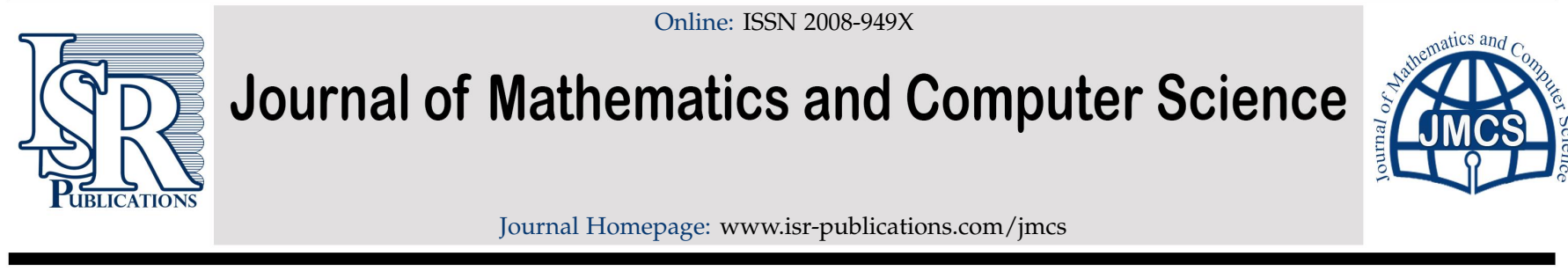

\title{
Global dynamics of delayed HIV infection models including impairment of B-cell functions
}

\author{
Ahmed Elaiw $^{\mathrm{a}, *}$, Safiya Alshehaiween ${ }^{\mathrm{a}, \mathrm{b}}$, Aatef Hobiny ${ }^{\mathrm{a}}$ \\ a Department of Mathematics, Faculty of Science, King Abdulaziz University, P. O. Box 80203, Jeddah 21589, Saudi Arabia. \\ ${ }^{b}$ Department of Mathematics, Faculty of Science, Taibah University, P. O. Box 344, Medina 42353, Saudi Arabia.
}

\begin{abstract}
In this paper, we construct delayed HIV dynamics models with impairment of B-cell functions. Two forms of the incidence rate have been considered, bilinear and general. Three types of infected cells and five-time delays have been incorporated into the models. The well-posedness of the models is justified. The models admit two equilibria, which are determined by the basic reproduction number $R_{0}$. The global stability of each equilibrium is proven by utilizing the Lyapunov function and LaSalle's invariance principle. Numerical simulations illustrate the theoretical results.
\end{abstract}

Keywords: HIV dynamics, global stability, Lyapunov function, B-cell impairment, latent reservoirs, time delay.

2010 MSC: 34D20, 34D23, 37N25, 92B05.

(C)2020 All rights reserved.

\section{Introduction}

Modeling the HIV dynamics has received considerable attention from mathematicians during the recent decades. A vast of mathematical models focused on exploring the relation between three main compartments, uninfected $\mathrm{CD} 4^{+} \mathrm{T}$ cells $(\mathrm{U})$, infected cells producing viruses (I), and HIV particles (P). The first HIV dynamics model was proposed by Nowak and Bangham [45] as:

$$
\begin{aligned}
\dot{\mathrm{U}}(\mathrm{t}) & =\rho-\gamma \mathrm{U}(\mathrm{t})-\omega \mathrm{U}(\mathrm{t}) \mathrm{P}(\mathrm{t}), \\
\dot{\mathrm{I}}(\mathrm{t}) & =\omega \mathrm{U}(\mathrm{t}) \mathrm{P}(\mathrm{t})-\beta \mathrm{I}(\mathrm{t}), \\
\dot{\mathrm{P}}(\mathrm{t}) & =\beta \mathrm{M}_{1} \mathrm{I}(\mathrm{t})-\xi \mathrm{P}(\mathrm{t}) .
\end{aligned}
$$

The production and death rate constants of compartments $(U, I, P)$ are give by $(\rho, \omega, \varkappa)$ and $(\gamma, \beta, \xi)$, respectively. The term $\omega \mathrm{U}(\mathrm{t}) \mathrm{P}(\mathrm{t})$ represents the incidence rate of infection, where $\omega$ is a positive constant. During the recent decades, much more modifications on the basic HIV dynamics model have

\footnotetext{
*Corresponding author

Email addresses: a_m_elaiw@yahoo.com (Ahmed Elaiw), safiya.f.sh@gmail.com (Safiya Alshehaiween), ahobany@kau.edu.sa (Aatef Hobiny)
}

doi: $10.22436 /$ jmcs.020.02.08

Received: 2019-06-18 Revised: 2019-08-26 Accepted: 2019-09-16 
been introduced (see e.g. [2-6, 8-30, 46, 47, 52, 54]). Time delay between the initial virus contacts an uninfected cell and the production of new active viruses plays an important role in virus dynamics modeling. Time delay has been incorporated into the virus dynamics models in several works (see e.g. $[6,13,14,32,35,36,38,39,44,48])$. Mathematical models which include time delays are more accurate representations of biology when compared to the models without considering time delays.

One of the most important extensions to model (1.1)-(1.3) is to incorporate the population of the B cells. The function of $\mathrm{B}$ cells is to produce antibodies which bind to virus particles and mark it as a foreign structure for elimination by other cells of the immune system [46]. The antibodies can neutralize viruses and protect the body from infection [40]. The basic virus dynamics model with B cell immune response has been presented by Murase et al. [43]. Wang and Zou [53] have proposed the following model which takes under consideration the time lag between the virus contacts an uninfected cell and the production of new mature viruses.

$$
\begin{aligned}
\dot{\mathrm{U}}(\mathrm{t}) & =\rho-\gamma \mathrm{U}(\mathrm{t})-\omega \mathrm{U}(\mathrm{t}) \mathrm{P}(\mathrm{t}), \\
\dot{\mathrm{I}}(\mathrm{t}) & =\omega \mathrm{U}\left(\mathrm{t}-\tau_{1}\right) \mathrm{P}\left(\mathrm{t}-\tau_{1}\right)-\beta \mathrm{I}(\mathrm{t}), \\
\dot{\mathrm{P}}(\mathrm{t}) & =\varkappa \mathrm{I}\left(\mathrm{t}-\tau_{2}\right)-\xi \mathrm{P}(\mathrm{t})-\rho \mathrm{P}(\mathrm{t}) \mathrm{C}(\mathrm{t}), \\
\dot{\mathrm{C}}(\mathrm{t}) & =\varepsilon \mathrm{P}(\mathrm{t}) \mathrm{C}(\mathrm{t})-\mu \mathrm{C}(\mathrm{t}),
\end{aligned}
$$

where $\mathrm{C}(\mathrm{t})$ is the concentration of $\mathrm{B}$ cells. The term $\rho \mathrm{P}(\mathrm{t}) \mathrm{C}(\mathrm{t})$ represents the neutralization rate of HIV particles. Parameters $\varepsilon$ and $\mu$ are the proliferation and natural death rate constants of $\mathrm{B}$ cells, respectively. Parameter $\tau_{1}$ represents the time between an HIV contacts an uninfected CD4 ${ }^{+} \mathrm{T}$ cell and the cell becomes infected. The immature virus needs time $\tau_{2}$ to be mature. Many delayed viral infection models are developed with B cell immune response (see e.g. [19, 37, 42, 50, 51]). Nowak and May [46] have assumed a linear term for immune stimulation: $\mathrm{B}$ cell abundance increases in response to free HIV particles at rate $\varepsilon P(t)$ and this leads to $\dot{C}(t)=\varepsilon P(t)-\mu C(t)$.

On the other hand, there are some factors affect the B-cell function and cause the impairment of the B cells $[1,4,7]$. These factors include malnutrition, tumors, cytotoxic drugs, irradiation, aging, trauma, some diseases, e.g. diabetes, and immunosuppression by microbes, e.g., malaria, measles virus but especially HIV [40]. However, all previous delayed HIV models that constructed with B cell immune response ignoring the B-cell impairment. Miao et al. [41] have proposed a virus dynamics model with humoral impairment, but they did not studied the global stability analysis of the model.

The objective of the present paper is to propose and analyze two delayed HIV dynamics models taking into account the impairment of B cell functions. The infected cells are supposed to divided into three classes, latently infected, short lived productively infected, and long lived productively infected. The linear immune response is considered. In the second model, the incidence rate is given by a general nonlinear function. The nonnegativity and boundedness of the solutions are proven. The global stability of all equilibria of the models are established by constructing Lyapunov functions.

\section{Delayed HIV infection model with B-cell impairment}

The first suggested delayed HIV infection model with B-cell impairment is given by:

$$
\begin{aligned}
\dot{\mathrm{U}}(\mathrm{t}) & =\rho-\gamma \mathrm{U}(\mathrm{t})-\left(\omega_{1}+\omega_{2}+\omega_{3}\right) \mathrm{U}(\mathrm{t}) \mathrm{P}(\mathrm{t}), \\
\dot{\mathrm{L}}(\mathrm{t}) & =e^{-\theta_{1} \tau_{1}} \omega_{1} \mathrm{U}\left(\mathrm{t}-\tau_{1}\right) \mathrm{P}\left(\mathrm{t}-\tau_{1}\right)-(\zeta+v) \mathrm{L}(\mathrm{t}), \\
\dot{\mathrm{I}}(\mathrm{t}) & =e^{-\theta_{2} \tau_{2}} \omega_{2} \mathrm{U}\left(\mathrm{t}-\tau_{2}\right) \mathrm{P}\left(\mathrm{t}-\tau_{2}\right)+v \mathrm{~L}(\mathrm{t})-\beta \mathrm{I}(\mathrm{t}), \\
\dot{\mathrm{O}}(\mathrm{t}) & =e^{-\theta_{3} \tau_{3}} \omega_{3} \mathrm{U}\left(\mathrm{t}-\tau_{3}\right) \mathrm{P}\left(\mathrm{t}-\tau_{3}\right)-\Lambda \mathrm{O}(\mathrm{t}), \\
\dot{\mathrm{P}}(\mathrm{t}) & =e^{-\theta_{4} \tau_{4}} \beta M_{1} \mathrm{I}\left(\mathrm{t}-\tau_{4}\right)+e^{-\theta_{5} \tau_{5}} \wedge M_{2} \mathrm{O}\left(\mathrm{t}-\tau_{5}\right)-\xi \mathrm{P}(\mathrm{t})-\rho \mathrm{P}(\mathrm{t}) \mathrm{C}(\mathrm{t}), \\
\dot{\mathrm{C}}(\mathrm{t}) & =\varepsilon \mathrm{P}(\mathrm{t})-\mu \mathrm{C}(\mathrm{t})-\vartheta \mathrm{P}(\mathrm{t}) \mathrm{C}(\mathrm{t}),
\end{aligned}
$$


where $L(t), I(t)$, and $O(t)$ represent, respectively, the concentration of the latently infected cells, short lived productively infected cells, and long lived productively infected cells at time t. The term $\left(\omega_{1}+\right.$ $\left.\omega_{2}+\omega_{3}\right) U(t) P(t)$, represents the incidence rate. Latently infected cells die with rate $\zeta \mathrm{L}(\mathrm{t})$ and they are transmitted to short lived productively infected cells with rate $v L(t)$. Parameters $M_{1}$ and $M_{2}$ are the average number of HIV particles generated in the lifetime of the short lived productively infected cells and long lived productively infected cells, respectively. Parameter $\Lambda$ is the natural death rate constant of the long lived productively infected cells. The B cell impairment rate is given by $\vartheta P(t) C(t)$. All previous described parameters are positive constants. Parameters $\tau_{1}, \tau_{2}$ and $\tau_{3}$ represent the times between HIV contacts an uninfected $\mathrm{CD} 4^{+} \mathrm{T}$ cell and the cell becomes latently infected, short lived productively infected and long lived productively infected, respectively. The factor $e^{-\theta_{k} \tau_{k}}, k=1,2,3$ represents the damage of $\mathrm{CD}^{+} \mathrm{T}$ cells during the interval $\left[t-\tau_{k}, t\right]$. The parameters $\tau_{4}$ and $\tau_{5}$ represent the time necessary for producing new mature HIV particles from the short lived productively infected cells and long lived productively infected cells, respectively. The factors $e^{-\theta_{4} \tau_{4}}$ and $e^{-\theta_{5} \tau_{5}}$ represent the loss of short lived productively infected cells and long lived productively infected cells during the intervals $\left[t-\tau_{4}, t\right]$ and $\left[t-\tau_{5}, t\right]$, respectively. Here, $\tau_{1}, \tau_{2}, \tau_{3}, \tau_{4}$, and $\tau_{5}$ are positive constants.

The initial conditions of model (2.1)-(2.6) are

$$
\begin{aligned}
\mathrm{U}(\mathrm{s}) & =\varphi_{1}(\mathrm{~s}), \quad \mathrm{L}(\mathrm{s})=\varphi_{2}(\mathrm{~s}), \\
\mathrm{I}(\mathrm{s}) & =\varphi_{3}(\mathrm{~s}), \quad \mathrm{O}(\mathrm{s})=\varphi_{4}(\mathrm{~s}), \\
\mathrm{P}(\mathrm{s}) & =\varphi_{5}(\mathrm{~s}), \quad \mathrm{C}(\mathrm{s})=\varphi_{6}(\mathrm{~s}), \\
\varphi_{\mathrm{i}}(\mathrm{s}) & \geqslant 0, \mathrm{~s} \in[-\bar{\tau}, 0], \quad i=1,2, \ldots, 6,
\end{aligned}
$$

where $\bar{\tau}=\max \left\{\tau_{1}, \tau_{2}, \tau_{3}, \tau_{4}, \tau_{5}\right\}$ and $\left(\varphi_{1}, \varphi_{2}, \ldots, \varphi_{6}\right) \in \mathcal{C}\left([-\bar{\tau}, 0], \mathbb{R}_{\geqslant 0}^{6}\right)$, where $\mathcal{C}$ is the Banach space of continuous function mapping the interval $[-\bar{\tau}, 0]$ into $\mathbb{R}_{\geqslant 0}^{6}$. By the standard theory of functional differential equations [31,34], we know that the system has a unique solution satisfying the initial conditions (2.7).

Proposition 2.1. Let $\mathrm{K}(\mathrm{t}, \varphi)$ be the solution of the model (2.1)-(2.6) with the initial conditions (2.7), then $\mathrm{U}(\mathrm{t})$, $\mathrm{L}(\mathrm{t}), \mathrm{I}(\mathrm{t}), \mathrm{O}(\mathrm{t}), \mathrm{P}(\mathrm{t})$, and $\mathrm{C}(\mathrm{t})$ are all non-negative and ultimately bounded for all $\mathrm{t} \geqslant 0$.

Proof. Assume that $\mathrm{U}(\mathrm{t})$ losses its positivity on some local existence interval $[0, j]$ for some constant $j$ and let $j^{*} \in[0, j]$ be such that $U\left(j^{*}\right)=0$. By Eq. (2.1), we have $\dot{U}\left(j^{*}\right)=\rho>0$. Hence $U(t)>0$ for some $t \in\left(j^{*}, j^{*}+k\right)$, where $k>0$ is sufficiently small. This leads to contradiction and thus $U(t)>0$, for all $t \geqslant 0$. Furthermore, for all $t \in[0, \bar{\tau}]$ we have

$$
\begin{aligned}
& \mathrm{L}(\mathrm{t})=\varphi_{2}(0) e^{-(\zeta+v) t}+e^{-\theta_{1} \tau_{1}} \omega_{1} \int_{0}^{t} e^{-(\zeta+v)(t-x)} \mathrm{U}\left(x-\tau_{1}\right) \mathrm{P}\left(x-\tau_{1}\right) \mathrm{d} x \geqslant 0, \\
& \mathrm{I}(\mathrm{t})=\varphi_{3}(0) e^{-\beta \mathrm{t}}+\int_{0}^{\mathrm{t}} e^{-\beta(t-x)}\left(e^{-\theta_{2} \tau_{2}} \omega_{2} \mathrm{U}\left(x-\tau_{2}\right) \mathrm{P}\left(x-\tau_{2}\right)+\nu \mathrm{L}(x)\right) \mathrm{d} x \geqslant 0, \\
& \mathrm{O}(\mathrm{t})=\varphi_{4}(0) e^{-\Lambda \mathrm{t}}+e^{-\theta_{3} \tau_{3}} \omega_{3} \int_{0}^{t} e^{-\Lambda(t-x)} \mathrm{U}\left(x-\tau_{3}\right) P\left(\xi-\tau_{3}\right) d x \geqslant 0, \\
& P(t)=\varphi_{5}(0) e^{-\int_{0}^{t}(\xi+\rho C(l)) d l}+\int_{0}^{t} e^{-\int_{x}^{t}(\xi+\rho C(l)) d l}\left(e^{-\theta_{4} \tau_{4}} \beta M_{1} I\left(x-\tau_{4}\right)+e^{-\theta_{5} \tau_{5}} \wedge M_{2} O\left(x-\tau_{5}\right)\right) d x \geqslant 0, \\
& C(t)=\varphi_{6}(0) e^{-\int_{0}^{t}(\mu+\vartheta P(l)) d l}+\varepsilon \int_{0}^{t} e^{-\int_{x}^{t}(\mu+\vartheta P(l)) d l} P(x) d x \geqslant 0 .
\end{aligned}
$$

Thus, by a recursive argument, we get $L(t), I(t), O(t), P(t), C(t) \geqslant 0$ for all $t \geqslant 0$. Hence, $\mathbb{R}_{\geqslant 0}^{6}$ is positively invariant for model (2.1)-(2.6). Next, from Eq. (2.1) we obtain $\dot{U}(t) \leqslant \rho-\gamma \mathrm{U}(\mathrm{t})$. This implies that $\lim _{\mathrm{t} \rightarrow \infty} \sup \mathrm{U}(\mathrm{t}) \leqslant \frac{\rho}{\gamma}$. Let

$$
F_{1}(t)=\frac{\omega_{1} e^{-\theta_{1} \tau_{1}}}{\omega_{1}+\omega_{2}+\omega_{3}} U\left(t-\tau_{1}\right)+\frac{\omega_{2} e^{-\theta_{2} \tau_{2}}}{\omega_{1}+\omega_{2}+\omega_{3}} U\left(t-\tau_{2}\right)+\frac{\omega_{3} e^{-\theta_{3} \tau_{3}}}{\omega_{1}+\omega_{2}+\omega_{3}} U\left(t-\tau_{3}\right)+L(t)+I(t)+O(t) .
$$


Then

$$
\begin{aligned}
\dot{\mathrm{F}}_{1}(\mathrm{t})= & \frac{\omega_{1} e^{-\theta_{1} \tau_{1}}}{\omega_{1}+\omega_{2}+\omega_{3}}\left[\rho-\gamma \mathrm{U}\left(\mathrm{t}-\tau_{1}\right)-\left(\omega_{1}+\omega_{2}+\omega_{3}\right) \mathrm{U}\left(\mathrm{t}-\tau_{1}\right) \mathrm{P}\left(\mathrm{t}-\tau_{1}\right)\right] \\
& +\frac{\omega_{2} e^{-\theta_{2} \tau_{2}}}{\omega_{1}+\omega_{2}+\omega_{3}}\left[\rho-\gamma \mathrm{U}\left(\mathrm{t}-\tau_{2}\right)-\left(\omega_{1}+\omega_{2}+\omega_{3}\right) \mathrm{U}\left(\mathrm{t}-\tau_{2}\right) \mathrm{P}\left(\mathrm{t}-\tau_{2}\right)\right] \\
& +\frac{\omega_{3} e^{-\theta_{3} \tau_{3}}}{\omega_{1}+\omega_{2}+\omega_{3}}\left[\rho-\gamma \mathrm{U}\left(\mathrm{t}-\tau_{3}\right)-\left(\omega_{1}+\omega_{2}+\omega_{3}\right) \mathrm{U}\left(\mathrm{t}-\tau_{3}\right) \mathrm{P}\left(\mathrm{t}-\tau_{3}\right)\right] \\
& +e^{-\theta_{1} \tau_{1}} \omega_{1} \mathrm{U}\left(\mathrm{t}-\tau_{1}\right) \mathrm{P}\left(\mathrm{t}-\tau_{1}\right)-(\zeta+v) \mathrm{L}(\mathrm{t})+e^{-\theta_{2} \tau_{2}} \omega_{2} \mathrm{U}\left(\mathrm{t}-\tau_{2}\right) \mathrm{P}\left(\mathrm{t}-\tau_{2}\right)+v \mathrm{~L}(\mathrm{t})-\beta \mathrm{I}(\mathrm{t}) \\
& +e^{-\theta_{3} \tau_{3}} \omega_{3} \mathrm{U}\left(\mathrm{t}-\tau_{3}\right) \mathrm{P}\left(\mathrm{t}-\tau_{3}\right)-\Lambda \mathrm{O}(\mathrm{t}) \\
= & \frac{\omega_{1} e^{-\theta_{1} \tau_{1}}}{\omega_{1}+\omega_{2}+\omega_{3}} \rho-\frac{\gamma \omega_{1} e^{-\theta_{1} \tau_{1}}}{\omega_{1}+\omega_{2}+\omega_{3}} \mathrm{U}\left(\mathrm{t}-\tau_{1}\right)+\frac{\omega_{2} e^{-\theta_{2} \tau_{2}}}{\omega_{1}+\omega_{2}+\omega_{3}} \rho-\frac{\gamma \omega_{2} e^{-\theta_{2} \tau_{2}}}{\omega_{1}+\omega_{2}+\omega_{3}} \mathrm{U}\left(\mathrm{t}-\tau_{2}\right) \\
& +\frac{\omega_{3} e^{-\theta_{3} \tau_{3}}}{\omega_{1}+\omega_{2}+\omega_{3}} \rho-\frac{\gamma \omega_{3} e^{-\theta_{3} \tau_{3}}}{\omega_{1}+\omega_{2}+\omega_{3}} \mathrm{U}\left(\mathrm{t}-\tau_{3}\right)-\zeta \mathrm{L}(\mathrm{t})-\beta \mathrm{I}(\mathrm{t})-\Lambda \mathrm{O}(\mathrm{t}) \\
\leqslant & \rho-\sigma_{1}\left[\frac{\omega_{1} e^{-\theta_{1} \tau_{1}}}{\omega_{1}+\omega_{2}+\omega_{3}} \mathrm{U}\left(\mathrm{t}-\tau_{1}\right)+\frac{\omega_{2} e^{-\theta_{2} \tau_{2}}}{\omega_{1}+\omega_{2}+\omega_{3}} \mathrm{U}\left(\mathrm{t}-\tau_{2}\right)\right. \\
& \left.+\frac{\omega_{3} e^{-\theta_{3} \tau_{3}}}{\omega_{1}+\omega_{2}+\omega_{3}} \mathrm{U}\left(\mathrm{t}-\tau_{3}\right)+\mathrm{L}(\mathrm{t})+\mathrm{I}(\mathrm{t})+\mathrm{O}(\mathrm{t})\right] \\
= & \rho-\sigma_{1} F_{1}(t),
\end{aligned}
$$

where $\sigma_{1}=\min \{\gamma, \zeta, \beta, \Lambda\}$. Hence, $\lim _{t \rightarrow \infty} \sup F_{1}(t) \leqslant s_{1}$, where $s_{1}=\frac{\rho}{\sigma_{1}}$. Since $U(t), L(t), I(t)$ and $O(t)$ are all non-negative, then $\lim _{t \rightarrow \infty} \sup L(t) \leqslant s_{1}, \limsup _{t \rightarrow \infty} I(t) \leqslant s_{1}$ and $\limsup _{t \rightarrow \infty} O(t) \leqslant s_{1}$ for all $t \geqslant 0$. Moreover, let $F_{2}(t)=P(t)+\frac{\xi}{2 \varepsilon} C(t)$. Then

$$
\begin{aligned}
\dot{\mathrm{F}}_{2}(t) & =e^{-\theta_{4} \tau_{4}} \beta M_{1} \mathrm{I}\left(\mathrm{t}-\tau_{4}\right)+e^{-\theta_{5} \tau_{5}} \wedge M_{2} \mathrm{O}\left(\mathrm{t}-\tau_{5}\right)-\xi \mathrm{P}(\mathrm{t})-\rho \mathrm{P}(\mathrm{t}) \mathrm{C}(\mathrm{t})+\frac{\xi}{2} \mathrm{P}(\mathrm{t})-\frac{\xi \mu}{2 \varepsilon} \mathrm{C}(\mathrm{t})-\frac{\xi \vartheta}{2 \varepsilon} \mathrm{P}(\mathrm{t}) \mathrm{C}(\mathrm{t}) \\
& =e^{-\theta_{4} \tau_{4}} \beta M_{1} \mathrm{I}\left(\mathrm{t}-\tau_{4}\right)+e^{-\theta_{5} \tau_{5}} \wedge M_{2} \mathrm{O}\left(\mathrm{t}-\tau_{5}\right)-\frac{\xi}{2} \mathrm{P}(\mathrm{t})-\frac{\xi \mu}{2 \varepsilon} \mathrm{C}(\mathrm{t})-\left(\rho+\frac{\xi \vartheta}{2 \varepsilon}\right) \mathrm{P}(\mathrm{t}) \mathrm{C}(\mathrm{t}) \\
& \leqslant \beta M_{1} \mathrm{I}\left(\mathrm{t}-\tau_{4}\right)+\Lambda M_{2} \mathrm{O}\left(\mathrm{t}-\tau_{5}\right)-\frac{\xi}{2} \mathrm{P}(\mathrm{t})-\frac{\xi \mu}{2 \varepsilon} \mathrm{C}(\mathrm{t}) \\
& \leqslant \beta M_{1} s_{1}+\Lambda M_{2} s_{1}-\frac{\xi}{2} \mathrm{P}(\mathrm{t})-\frac{\xi \mu}{2 \varepsilon} \mathrm{C}(\mathrm{t}) \\
& \leqslant \beta M_{1} s_{1}+\Lambda M_{2} s_{1}-\sigma_{2} \mathrm{~F}_{2}(\mathrm{t})
\end{aligned}
$$

where $\sigma_{2}=\min \left\{\frac{\xi}{2}, \mu\right\}$. Hence, $\lim _{t \rightarrow \infty} \sup _{2}(t) \leqslant s_{2}$, where $s_{2}=\frac{\beta M_{1} s_{1}+\Lambda M_{2} s_{1}}{\sigma_{2}}$. The non-negativity of $P(t)$ and $C(t)$ implies $\lim _{t \rightarrow \infty} \sup P(t) \leqslant s_{2}$ and $\lim _{t \rightarrow \infty} \sup C(t) \leqslant s_{3}$, where $s_{3}=\frac{2 \varepsilon s_{2}}{\xi}$.

The basic reproduction number for model (2.1)-(2.6) is defined as

$$
R_{0}=\frac{\rho\left[\omega_{1} v M_{1} e^{-\theta_{1} \tau_{1}-\theta_{4} \tau_{4}}+(\zeta+v)\left(\omega_{2} M_{1} e^{-\theta_{2} \tau_{2}-\theta_{4} \tau_{4}}+\omega_{3} M_{2} e^{-\theta_{3} \tau_{3}-\theta_{5} \tau_{5}}\right)\right]}{\xi \gamma(\zeta+v)} .
$$

Lemma 2.2. Consider model (2.1)-(2.6), then

(i) if $\mathrm{R}_{0} \leqslant 1$, then the model has only one equilibrium point $\mathrm{EP}_{0}$;

(ii) if $\mathrm{R}_{0}>1$, then the model has two equilibria $\mathrm{EP}_{0}$ and $\mathrm{EP}_{1}$. 
Proof. At any equilibrium $\mathrm{EP}(\mathrm{U}, \mathrm{L}, \mathrm{I}, \mathrm{O}, \mathrm{P}, \mathrm{C})$ we have

$$
\begin{aligned}
& \rho-\gamma \mathrm{U}-\left(\omega_{1}+\omega_{2}+\omega_{3}\right) \mathrm{UP}=0, \\
& e^{-\theta_{1} \tau_{1}} \omega_{1} \mathrm{UP}-(\zeta+v) \mathrm{L}=0, \\
& e^{-\theta_{2} \tau_{2}} \omega_{2} \mathrm{UP}+v \mathrm{~L}-\beta \mathrm{I}=0, \\
& e^{-\theta_{3} \tau_{3}} \omega_{3} \mathrm{UP}-\wedge \mathrm{O}=0, \\
& e^{-\theta_{4} \tau_{4}} \beta M_{1} \mathrm{I}+e^{-\theta_{5} \tau_{5}} \wedge M_{2} \mathrm{O}-\xi \mathrm{P}-\rho \mathrm{PC}=0, \\
& \mathcal{P}-\mu \mathrm{C}-\vartheta \mathrm{PC}=0 .
\end{aligned}
$$

From equations (2.8)-(2.13) we get an infection-free equilibrium $\mathrm{EP}_{0}=\left(\mathrm{U}_{0}, 0,0,0,0,0\right)$, where $\mathrm{U}_{0}=\frac{\rho}{\gamma}$ and a unique endemic equilibrium $\mathrm{EP}_{1}=\left(\mathrm{U}_{1}, \mathrm{~L}_{1}, \mathrm{I}_{1}, \mathrm{O}_{1}, \mathrm{P}_{1}, \mathrm{C}_{1}\right)$, where

$$
\begin{aligned}
\mathrm{U}_{1} & =\frac{\rho}{\gamma+\left(\omega_{1}+\omega_{2}+\omega_{3}\right) \mathrm{P}_{1}}, & \mathrm{~L}_{1} & =\frac{e^{-\theta_{1} \tau_{1}} \rho \omega_{1} \mathrm{P}_{1}}{(\zeta+v)\left(\gamma+\left(\omega_{1}+\omega_{2}+\omega_{3}\right) \mathrm{P}_{1}\right)}, \\
\mathrm{I}_{1} & =\frac{\rho\left(e^{-\theta_{2} \tau_{2}} \omega_{2}(\zeta+v)+e^{-\theta_{1} \tau_{1}} v \omega_{1}\right) \mathrm{P}_{1}}{\beta(\zeta+v)\left(\gamma+\left(\omega_{1}+\omega_{2}+\omega_{3}\right) \mathrm{P}_{1}\right)}, & \mathrm{O}_{1} & =\frac{e^{-\theta_{3} \tau_{3}} \rho \omega_{3} \mathrm{P}_{1}}{\Lambda\left(\gamma+\left(\omega_{1}+\omega_{2}+\omega_{3}\right) \mathrm{P}_{1}\right)}, \\
\mathrm{P}_{1} & =\frac{-\mathrm{b}+\sqrt{\mathrm{b}^{2}-4 a c}}{2 \mathrm{a}}, & \mathrm{C}_{1} & =\frac{\varepsilon \mathrm{P}_{1}}{\vartheta \mathrm{P}_{1}+\mu},
\end{aligned}
$$

where

$$
\begin{aligned}
a= & \frac{1}{\gamma^{3} \mu^{2}(\zeta+v)}\left[e^{-\theta_{1} \tau_{1}-\theta_{4} \tau_{4}} v \rho \mu^{2} M_{1} \omega_{1}^{3}+2 e^{-\theta_{1} \tau_{1}-\theta_{4} \tau_{4}} v \rho \mu^{2} M_{1} \omega_{1} \omega_{2} \omega_{3}+e^{-\theta_{1} \tau_{1}-\theta_{4} \tau_{4}} v \rho \mu^{2} M_{1} \omega_{1}\left(\omega_{2}^{2}+\omega_{3}^{2}\right)\right. \\
& +2 e^{-\theta_{1} \tau_{1}-\theta_{4} \tau_{4}} v \rho \mu^{2} M_{1} \omega_{1}^{2}\left(\omega_{2}+\omega_{3}\right)+(\zeta+v)\left(e^{-\theta_{3} \tau_{3}-\theta_{5} \tau_{5}} \rho \mu^{2} M_{2} \omega_{3}^{3}+2 e^{-\theta_{2} \tau_{2}-\theta_{4} \tau_{4}} \rho \mu^{2} M_{1} \omega_{1} \omega_{2}^{2}\right. \\
& +2 e^{-\theta_{3} \tau_{3}-\theta_{5} \tau_{5}} \rho \mu^{2} M_{2} \omega_{1} \omega_{3}^{2}+e^{-\theta_{2} \tau_{2}-\theta_{4} \tau_{4}} \rho \mu^{2} M_{1} \omega_{2} \omega_{3}^{2}+2 e^{-\theta_{3} \tau_{3}-\theta_{5} \tau_{5}} \rho \mu^{2} M_{2} \omega_{2} \omega_{3}^{2} \\
& +e^{-\theta_{2} \tau_{2}-\theta_{4} \tau_{4}} \rho \mu^{2} M_{1} \omega_{1}^{2} \omega_{2}+e^{-\theta_{3} \tau_{3}-\theta_{5} \tau_{5}} \rho \mu^{2} M_{2} \omega_{1}^{2} \omega_{3}+2 e^{-\theta_{2} \tau_{2}-\theta_{4} \tau_{4}} \rho \mu^{2} M_{1} \omega_{2}^{2} \omega_{3} \\
& +e^{-\theta_{3} \tau_{3}-\theta_{5} \tau_{5}} \rho \mu^{2} M_{2} \omega_{2}^{2} \omega_{3}+2 e^{-\theta_{2} \tau_{2}-\theta_{4} \tau_{4}} \rho \mu^{2} M_{1} \omega_{1} \omega_{2} \omega_{3}+2 e^{-\theta_{3} \tau_{3}-\theta_{5} \tau_{5}} \rho \mu^{2} M_{2} \omega_{1} \omega_{2} \omega_{3} \\
+ & \left.\left.e^{-\theta_{2} \tau_{2}-\theta_{4} \tau_{4}} \rho \mu^{2} M_{1} \omega_{2}^{3}+\vartheta \varepsilon \rho \gamma^{3}\right)\right] \\
\mathrm{b}= & -\frac{1}{\gamma^{2} \mu(\zeta+v)}\left[\gamma^{2} \varepsilon \rho(\zeta+v)\right. \\
& +\omega_{3} \rho \mu M_{2} e^{-\theta_{3} \tau_{3}-\theta_{5} \tau_{5}}(\zeta+v)\left(\omega_{1}+\omega_{3}\right)+\omega_{2} \omega_{3} \rho \mu(\zeta+v)\left(e^{-\theta_{2} \tau_{2}-\theta_{4} \tau_{4}} M_{1}+e^{-\theta_{3} \tau_{3}-\theta_{5} \tau_{5}} M_{2}\right) \\
& \left.+\omega_{2} \rho \mu M_{1} e^{-\theta_{2} \tau_{2}-\theta_{4} \tau_{4}}(\zeta+v)\left(\omega_{1}+\omega_{2}\right)+\omega_{1} \rho \mu v M_{1} e^{-\theta_{1} \tau_{1}-\theta_{4} \tau_{4}}\left(\omega_{1}+\omega_{2}+\omega_{3}\right)\right] \\
c= & \xi\left(1-R_{0}\right) .
\end{aligned}
$$

Therefore, if $R_{0}>1$, then $c<0$ and $P_{1}>0$. Then the equilibrium $E P_{1}$ exists when $R_{0}>1$.

\subsection{Global stability of equilibria}

Define the function $G(u)=u-1-\ln u$. Clearly, $G(u) \geqslant 0$, for $u>0$ and $G(1)=0$.

Theorem 2.3. Let $\mathrm{R}_{0}<1$, then $\mathrm{EP}_{0}$ of model (2.1)-(2.6) is globally asymptotically stable (G.A.S).

Proof. Construct a Lyapunov function $\mathrm{W}_{0}(\mathrm{U}(\mathrm{t}), \mathrm{L}(\mathrm{t}), \mathrm{I}(\mathrm{t}), \mathrm{O}(\mathrm{t}), \mathrm{P}(\mathrm{t}), \mathrm{C}(\mathrm{t}))$ as:

$$
\begin{aligned}
W_{0}= & \hbar U_{0} G\left(\frac{U(t)}{U_{0}}\right)+\frac{v M_{1} e^{-\theta_{4} \tau_{4}}}{\zeta+v} L(t)+M_{1} e^{-\theta_{4} \tau_{4}} I(t)+M_{2} e^{-\theta_{5} \tau_{5}} O(t)+P(t)+\frac{\xi}{\varepsilon}\left(1-R_{0}\right) C(t) \\
& +\frac{v M_{1} \omega_{1} e^{-\theta_{1} \tau_{1}-\theta_{4} \tau_{4}}}{\zeta+v} \int_{t-\tau_{1}}^{t} U(s) P(s) d s+M_{1} \omega_{2} e^{-\theta_{2} \tau_{2}-\theta_{4} \tau_{4}} \int_{t-\tau_{2}}^{t} U(s) P(s) d s
\end{aligned}
$$




$$
+M_{2} \omega_{3} e^{-\theta_{3} \tau_{3}-\theta_{5} \tau_{5}} \int_{t-\tau_{3}}^{t} U(s) P(s) d s+\beta M_{1} e^{-\theta_{4} \tau_{4}} \int_{t-\tau_{4}}^{t} I(s) d s+\Lambda M_{2} e^{-\theta_{5} \tau_{5}} \int_{t-\tau_{5}}^{t} O(s) d s,
$$

where

$$
\hbar=\frac{1}{\omega_{1}+\omega_{2}+\omega_{3}}\left(\frac{v M_{1} \omega_{1} e^{-\theta_{1} \tau_{1}-\theta_{4} \tau_{4}}}{\zeta+v}+M_{1} \omega_{2} e^{-\theta_{2} \tau_{2}-\theta_{4} \tau_{4}}+M_{2} \omega_{3} e^{-\theta_{3} \tau_{3}-\theta_{5} \tau_{5}}\right) .
$$

Calculating $\frac{d W_{0}}{d t}$, we get

$$
\begin{aligned}
& \frac{d W_{0}}{d t}=\hbar\left(1-\frac{U_{0}}{U(t)}\right)\left(\rho-\gamma \mathrm{U}(t)-\left(\omega_{1}+\omega_{2}+\omega_{3}\right) U(t) P(t)\right) \\
& +\frac{v M_{1} e^{-\theta_{4} \tau_{4}}}{\zeta+v}\left(e^{-\theta_{1} \tau_{1}} \omega_{1} u\left(t-\tau_{1}\right) P\left(t-\tau_{1}\right)-(\zeta+v) L(t)\right) \\
& +M_{1} e^{-\theta_{4} \tau_{4}}\left(e^{-\theta_{2} \tau_{2}} \omega_{2} \mathrm{U}\left(\mathrm{t}-\tau_{2}\right) \mathrm{P}\left(\mathrm{t}-\tau_{2}\right)+v \mathrm{~L}(\mathrm{t})-\beta \mathrm{I}(\mathrm{t})\right) \\
& +M_{2} e^{-\theta_{5} \tau_{5}}\left(e^{-\theta_{3} \tau_{3}} \omega_{3} \mathrm{U}\left(\mathrm{t}-\tau_{3}\right) \mathrm{P}\left(\mathrm{t}-\tau_{3}\right)-\Lambda \mathrm{O}(\mathrm{t})\right) \\
& +\beta M_{1} e^{-\theta_{4} \tau_{4}} \mathrm{I}\left(\mathrm{t}-\tau_{4}\right)+\Lambda M_{2} e^{-\theta_{5} \tau_{5}} \mathrm{O}\left(\mathrm{t}-\tau_{5}\right)-\xi \mathrm{P}(\mathrm{t})-\rho \mathrm{P}(\mathrm{t}) \mathrm{C}(\mathrm{t}) \\
& +\frac{\xi}{\varepsilon}\left(1-R_{0}\right)(\varepsilon P(t)-\mu \mathrm{C}(\mathrm{t})-\vartheta \mathrm{P}(\mathrm{t}) \mathrm{C}(\mathrm{t})) \\
& +\frac{v M_{1} \omega_{1} e^{-\theta_{1} \tau_{1}-\theta_{4} \tau_{4}}}{\zeta+v}\left(\mathrm{U}(\mathrm{t}) \mathrm{P}(\mathrm{t})-\mathrm{U}\left(\mathrm{t}-\tau_{1}\right) \mathrm{P}\left(\mathrm{t}-\tau_{1}\right)\right) \\
& +M_{1} \omega_{2} e^{-\theta_{2} \tau_{2}-\theta_{4} \tau_{4}}\left(\mathrm{U}(\mathrm{t}) \mathrm{P}(\mathrm{t})-\mathrm{U}\left(\mathrm{t}-\tau_{2}\right) \mathrm{P}\left(\mathrm{t}-\tau_{2}\right)\right) \\
& +M_{2} \omega_{3} e^{-\theta_{3} \tau_{3}-\theta_{5} \tau_{5}}\left(\mathrm{U}(\mathrm{t}) \mathrm{P}(\mathrm{t})-\mathrm{U}\left(\mathrm{t}-\tau_{3}\right) \mathrm{P}\left(\mathrm{t}-\tau_{3}\right)\right) \\
& +\beta M_{1} e^{-\theta_{4} \tau_{4}}\left(\mathrm{I}(\mathrm{t})-\mathrm{I}\left(\mathrm{t}-\tau_{4}\right)\right)+\Lambda M_{2} e^{-\theta_{5} \tau_{5}}\left(\mathrm{O}(\mathrm{t})-\mathrm{O}\left(\mathrm{t}-\tau_{5}\right)\right) \\
& =\hbar\left(1-\frac{\mathrm{U}_{0}}{\mathrm{U}(\mathrm{t})}\right)(\rho-\gamma \mathrm{U}(\mathrm{t}))+\hbar\left(\omega_{1}+\omega_{2}+\omega_{3}\right) \mathrm{U}_{0} \mathrm{P}(\mathrm{t})-\xi \mathrm{P}(\mathrm{t})-\rho \mathrm{P}(\mathrm{t}) \mathrm{C}(\mathrm{t})+\xi\left(1-\mathrm{R}_{0}\right) \mathrm{P}(\mathrm{t}) \\
& -\frac{\xi \mu}{\varepsilon}\left(1-R_{0}\right) C(t)-\frac{\xi \vartheta}{\varepsilon}\left(1-R_{0}\right) P(t) C(t) .
\end{aligned}
$$

We have $\xi R_{0}=\hbar U_{0}\left(\omega_{1}+\omega_{2}+\omega_{3}\right)$, then

$$
\hbar\left(\omega_{1}+\omega_{2}+\omega_{3}\right) U_{0} P(t)-\xi P(t)+\xi\left(1-R_{0}\right) P(t)=0 .
$$

Then

$$
\frac{d W_{0}}{d t}=\frac{-\hbar \gamma\left(U(t)-U_{0}\right)^{2}}{U(t)}-\frac{\xi \mu}{\varepsilon}\left(1-R_{0}\right) C(t)-\left(\rho+\frac{\xi \vartheta}{\varepsilon}\left(1-R_{0}\right)\right) P(t) C(t) .
$$

Since $R_{0}<1$, then for all $U(t), P(t), C(t)>0$ we have $\frac{d W_{0}}{d t} \leqslant 0$. Moreover, $\frac{d W_{0}}{d t}=0$ when $U(t)=U_{0}$ and $C(t)=0$. Let $D_{0}=\left\{(U(t), L(t), I(t), O(t), P(t), C(t)): \frac{d W_{0}}{d t}=0\right\}$ and $N_{0}$ be the largest invariant subset of $D_{0}$. The trajectory of model (2.1)-(2.6) tend to $N_{0}$ [31]. All the elements of $N_{0}$ satisfy $U(t)=U_{0}$ and $\mathrm{C}(\mathrm{t})=0$. Then Eq. (2.6) yields

$$
\dot{\mathrm{C}}(\mathrm{t})=0=\varepsilon \mathrm{P}(\mathrm{t}) \quad \Longrightarrow \quad \mathrm{P}(\mathrm{t})=0, \quad \text { for all } \mathrm{t} .
$$

Also from Eq. (2.5) we get

$$
0=\dot{P}(t)=e^{-\theta_{4} \tau_{4}} \beta M_{1} I\left(t-\tau_{4}\right)+e^{-\theta_{5} \tau_{5}} \wedge M_{2} \mathrm{O}\left(t-\tau_{5}\right) .
$$

The nonnegativity of $I$ and $O$ implies that $I(t)=O(t)=0$ for all $t$. Then from Eq. (2.3), we have $0=\dot{I}(t)=v L(t)$. It follows that, $L(t)=0$ for all $t$. Hence, $N_{0}=\left\{E P_{0}\right\}$. From LaSalle's invariance principle (L.I.P), we derive that if $\mathrm{R}_{0}<1$, then $\mathrm{EP}_{0}$ is G.A.S. 
Theorem 2.4. Let $\mathrm{R}_{0}>1$, then $\mathrm{EP}_{1}$ of model (2.1)-(2.6) is G.A.S.

Proof. Define $\mathrm{W}_{11}(\mathrm{U}(\mathrm{t}), \mathrm{L}(\mathrm{t}), \mathrm{I}(\mathrm{t}), \mathrm{O}(\mathrm{t}), \mathrm{P}(\mathrm{t}), \mathrm{C}(\mathrm{t}))$ as:

$$
\begin{aligned}
W_{11}= & \hbar U_{1} G\left(\frac{U(t)}{U_{1}}\right)+\frac{v M_{1} e^{-\theta_{4} \tau_{4}}}{\zeta+v} L_{1} G\left(\frac{L(t)}{L_{1}}\right)+M_{1} e^{-\theta_{4} \tau_{4}} I_{1} G\left(\frac{I(t)}{I_{1}}\right)+M_{2} e^{-\theta_{5} \tau_{5}} O_{1} G\left(\frac{O(t)}{O_{1}}\right) \\
& +P_{1} G\left(\frac{P(t)}{P_{1}}\right)+\frac{\rho}{2\left(\varepsilon-\vartheta C_{1}\right)}\left(C(t)-C_{1}\right)^{2} .
\end{aligned}
$$

Note that from the equilibrium condition (2.13) that

$$
\varepsilon-\vartheta \mathrm{C}_{1}=\frac{\mu \mathrm{C}_{1}}{\mathrm{P}_{1}}>0
$$

Calculating $\frac{d W_{11}}{d t}$, we obtain

$$
\begin{aligned}
\frac{d W_{11}}{d t}= & \hbar\left(1-\frac{U_{1}}{U(t)}\right)\left(\rho-\gamma \mathrm{U}(t)-\left(\omega_{1}+\omega_{2}+\omega_{3}\right) \mathrm{U}(\mathrm{t}) \mathrm{P}(\mathrm{t})\right) \\
& +\frac{v M_{1} e^{-\theta_{4} \tau_{4}}}{\zeta+v}\left(1-\frac{\mathrm{L}_{1}}{\mathrm{~L}(\mathrm{t})}\right)\left(e^{-\theta_{1} \tau_{1}} \omega_{1} \mathrm{U}\left(\mathrm{t}-\tau_{1}\right) \mathrm{P}\left(\mathrm{t}-\tau_{1}\right)-(\zeta+v) \mathrm{L}(\mathrm{t})\right) \\
& +M_{1} e^{-\theta_{4} \tau_{4}}\left(1-\frac{\mathrm{I}_{1}}{\mathrm{I}(\mathrm{t})}\right)\left(e^{-\theta_{2} \tau_{2}} \omega_{2} \mathrm{U}\left(\mathrm{t}-\tau_{2}\right) \mathrm{P}\left(\mathrm{t}-\tau_{2}\right)+v \mathrm{~L}(\mathrm{t})-\beta \mathrm{I}(\mathrm{t})\right) \\
& +M_{2} e^{-\theta_{5} \tau_{5}}\left(1-\frac{\mathrm{O}_{1}}{\mathrm{O}(\mathrm{t})}\right)\left(e^{-\theta_{3} \tau_{3}} \omega_{3} \mathrm{U}\left(\mathrm{t}-\tau_{3}\right) \mathrm{P}\left(\mathrm{t}-\tau_{3}\right)-\Lambda \mathrm{O}(\mathrm{t})\right) \\
& +\left(1-\frac{\mathrm{P}_{1}}{\mathrm{P}(\mathrm{t})}\right)\left(e^{-\theta_{4} \tau_{4}} \beta M_{1} \mathrm{I}\left(\mathrm{t}-\tau_{4}\right)+e^{-\theta_{5} \tau_{5}} \wedge M_{2} \mathrm{O}\left(\mathrm{t}-\tau_{5}\right)-\xi \mathrm{P}(\mathrm{t})-\rho \mathrm{P}(\mathrm{t}) \mathrm{C}(\mathrm{t})\right) \\
& +\frac{\rho}{\varepsilon-\vartheta \mathrm{C}_{1}}\left(\mathrm{C}(\mathrm{t})-\mathrm{C}_{1}\right)(\varepsilon \mathrm{P}(\mathrm{t})-\mu \mathrm{C}(\mathrm{t})-\vartheta \mathrm{P}(\mathrm{t}) \mathrm{C}(\mathrm{t})) .
\end{aligned}
$$

Define $W_{12}(\mathrm{U}(\mathrm{t}), \mathrm{L}(\mathrm{t}), \mathrm{I}(\mathrm{t}), \mathrm{O}(\mathrm{t}), \mathrm{P}(\mathrm{t}), \mathrm{C}(\mathrm{t}))$ as:

$$
\begin{aligned}
W_{12}= & \frac{v M_{1} \omega_{1} e^{-\theta_{1} \tau_{1}-\theta_{4} \tau_{4}}}{\zeta+v} U_{1} P_{1} \int_{t-\tau_{1}}^{t} G\left(\frac{U(s) p(s)}{U_{1} P_{1}}\right) d s \\
& +M_{1} \omega_{2} e^{-\theta_{2} \tau_{2}-\theta_{4} \tau_{4}} U_{1} P_{1} \int_{t-\tau_{2}}^{t} G\left(\frac{U(s) p(s)}{U_{1} P_{1}}\right) d s \\
& +M_{2} \omega_{3} e^{-\theta_{3} \tau_{3}-\theta_{5} \tau_{5}} U_{1} P_{1} \int_{t-\tau_{3}}^{t} G\left(\frac{U(s) p(s)}{U_{1} P_{1}}\right) d s
\end{aligned}
$$

Calculating $\frac{d W_{12}}{d t}$, we get

$$
\begin{aligned}
\frac{d W_{12}}{d t}= & \frac{v M_{1} \omega_{1} e^{-\theta_{1} \tau_{1}-\theta_{4} \tau_{4}}}{\zeta+v} U_{1} P_{1}\left[\frac{U(t) P(t)}{U_{1} P_{1}}-\frac{U\left(t-\tau_{1}\right) P\left(t-\tau_{1}\right)}{U_{1} P_{1}}+\ln \left(\frac{U\left(t-\tau_{1}\right) P\left(t-\tau_{1}\right)}{U(t) P(t)}\right)\right] \\
& +M_{1} \omega_{2} e^{-\theta_{2} \tau_{2}-\theta_{4} \tau_{4}} U_{1} P_{1}\left[\frac{U(t) P(t)}{U_{1} P_{1}}-\frac{U\left(t-\tau_{2}\right) P\left(t-\tau_{2}\right)}{U_{1} P_{1}}+\ln \left(\frac{U\left(t-\tau_{2}\right) P\left(t-\tau_{2}\right)}{U(t) P(t)}\right)\right] \\
& +M_{2} \omega_{3} e^{-\theta_{3} \tau_{3}-\theta_{5} \tau_{5}} U_{1} P_{1}\left[\frac{U(t) P(t)}{U_{1} P_{1}}-\frac{U\left(t-\tau_{3}\right) P\left(t-\tau_{3}\right)}{U_{1} P_{1}}+\ln \left(\frac{U\left(t-\tau_{3}\right) P\left(t-\tau_{3}\right)}{U(t) P(t)}\right)\right] .
\end{aligned}
$$

Define $W_{13}(\mathrm{U}(\mathrm{t}), \mathrm{L}(\mathrm{t}), \mathrm{I}(\mathrm{t}), \mathrm{P}(\mathrm{t}), \mathrm{P}(\mathrm{t}), \mathrm{C}(\mathrm{t}))$ as:

$$
W_{13}=\beta M_{1} e^{-\theta_{4} \tau_{4}} I_{1} \int_{t-\tau_{4}}^{t} G\left(\frac{I(s)}{I_{1}}\right) d s+\Lambda M_{2} e^{-\theta_{5} \tau_{5}} O_{1} \int_{t-\tau_{5}}^{t} G\left(\frac{O(s)}{O_{1}}\right) d s
$$


Calculating $\frac{d W_{13}}{d t}$ as:

$$
\begin{aligned}
\frac{d W_{13}}{d t}= & \beta M_{1} e^{-\theta_{4} \tau_{4}} I_{1}\left[\frac{I(t)}{I_{1}}-\frac{I\left(t-\tau_{4}\right)}{I_{1}}+\ln \left(\frac{I\left(t-\tau_{4}\right)}{I(t)}\right)\right] \\
& +\wedge M_{2} e^{-\theta_{5} \tau_{5}} O_{1}\left[\frac{O(t)}{O_{1}}-\frac{O\left(t-\tau_{5}\right)}{O_{1}}+\ln \left(\frac{O\left(t-\tau_{5}\right)}{O(t)}\right)\right] .
\end{aligned}
$$

Construct a Lyapunov function $\mathrm{W}_{1}(\mathrm{U}(\mathrm{t}), \mathrm{L}(\mathrm{t}), \mathrm{I}(\mathrm{t}), \mathrm{O}(\mathrm{t}), \mathrm{P}(\mathrm{t}), \mathrm{C}(\mathrm{t}))$ as

$$
W_{1}=W_{11}+W_{12}+W_{13}
$$

Then

$$
\frac{d W_{1}}{d t}=\frac{d W_{11}}{d t}+\frac{d W_{12}}{d t}+\frac{d W_{13}}{d t}
$$

Now we have

$$
\begin{aligned}
\frac{d W_{1}}{d t}= & \hbar\left(1-\frac{U_{1}}{U(t)}\right)(\rho-\gamma U(t))+\hbar\left(\omega_{1}+\omega_{2}+\omega_{3}\right) U_{1} P(t) \\
& -\frac{v M_{1} \omega_{1} e^{-\theta_{1} \tau_{1}-\theta_{4} \tau_{4}}}{\zeta+v} U\left(t-\tau_{1}\right) P\left(t-\tau_{1}\right) \frac{L_{1}}{L(t)}+v M_{1} e^{-\theta_{4} \tau_{4}} L_{1} \\
& -M_{1} \omega_{2} e^{-\theta_{2} \tau_{2}-\theta_{4} \tau_{4}} U\left(t-\tau_{2}\right) P\left(t-\tau_{2}\right) \frac{I_{1}}{I(t)}-v M_{1} e^{-\theta_{4} \tau_{4}} L(t) \frac{I_{1}}{I(t)}+\beta M_{1} e^{-\theta_{4} \tau_{4}} I_{1} \\
& -M_{2} \omega_{3} e^{-\theta_{3} \tau_{3}-\theta_{5} \tau_{5}} U\left(t-\tau_{3}\right) P\left(t-\tau_{3}\right) \frac{O_{1}}{O(t)}+\Lambda M_{2} e^{-\theta_{4} \tau_{4}} O_{1} \\
& -\beta M_{1} e^{-\theta_{4} \tau_{4}} I\left(t-\tau_{4}\right) \frac{P_{1}}{P(t)}-\Lambda M_{2} e^{-\theta_{5} \tau_{5}} O\left(t-\tau_{5}\right) \frac{P_{1}}{P(t)}-\xi\left(P(t)-P_{1}\right) \\
& -\rho\left(P(t)-P_{1}\right) C(t)+\frac{\rho}{\varepsilon-\vartheta C_{1}}\left(C(t)-C_{1}\right)(\varepsilon P(t)-\mu C(t)-\vartheta P(t) C(t)) \\
& +\frac{v M_{1} \omega_{1} e^{-\theta_{1} \tau_{1}-\theta_{4} \tau_{4}}}{\zeta+v} U_{1} P_{1} \ln \left(\frac{U\left(t-\tau_{1}\right) P\left(t-\tau_{1}\right)}{U(t) P(t)}\right) \\
& +M_{1} \omega_{2} e^{-\theta_{2} \tau_{2}-\theta_{4} \tau_{4}} U_{1} P_{1} \ln \left(\frac{U\left(t-\tau_{2}\right) P\left(t-\tau_{2}\right)}{U(t) P(t)}\right) \\
& +M_{2} \omega_{3} e^{-\theta_{3} \tau_{3}-\theta_{5} \tau_{5}} U_{1} P_{1} \ln \left(\frac{U\left(t-\tau_{3}\right) P\left(t-\tau_{3}\right)}{U(t) P(t)}\right) \\
& +\beta M_{1} e^{-\theta_{4} \tau_{4}} I_{1} \ln \left(\frac{I\left(t-\tau_{4}\right)}{I(t)}\right)+\Lambda M_{2} e^{-\theta_{5} \tau_{5}} O_{1} \ln \left(\frac{O\left(t-\tau_{5}\right)}{O(t)}\right)
\end{aligned}
$$

From the equilibrium conditions, we have:

$$
\begin{aligned}
\rho & =\gamma \mathrm{U}_{1}+\left(\omega_{1}+\omega_{2}+\omega_{3}\right) \mathrm{U}_{1} \mathrm{P}_{1}, \\
\frac{v M_{1} \omega_{1} e^{-\theta_{1} \tau_{1}-\theta_{4} \tau_{4}}}{\zeta+v} \mathrm{U}_{1} \mathrm{P}_{1} & =v M_{1} e^{-\theta_{4} \tau_{4}} \mathrm{~L}_{1}, \\
\beta M_{1} e^{-\theta_{4} \tau_{4}} \mathrm{I}_{1} & =\mathrm{M}_{1} \omega_{2} e^{-\theta_{2} \tau_{2}-\theta_{4} \tau_{4}} \mathrm{U}_{1} \mathrm{P}_{1}+v \mathrm{M}_{1} e^{-\theta_{4} \tau_{4}} \mathrm{~L}_{1}, \\
\mathrm{M}_{2} \omega_{3} e^{-\theta_{3} \tau_{3}-\theta_{5} \tau_{5}} \mathrm{U}_{1} \mathrm{P}_{1} & =\wedge M_{2} e^{-\theta_{5} \tau_{5}} \mathrm{O}_{1}, \\
\xi \mathrm{P}_{1}+\rho \mathrm{P}_{1} \mathrm{C}_{1} & =e^{-\theta_{4} \tau_{4}} \beta M_{1} \mathrm{I}_{1}+e^{-\theta_{5} \tau_{5}} \wedge M_{2} \mathrm{O}_{1}, \\
\varepsilon \mathrm{P}_{1} & =\mu \mathrm{C}_{1}+\vartheta \mathrm{P}_{1} \mathrm{C}_{1} .
\end{aligned}
$$

Utilizing the equilibrium conditions for $\mathrm{EP}_{1}$, we get

$$
\frac{d W_{1}}{d t}=\hbar\left(1-\frac{U_{1}}{\mathrm{U}(\mathrm{t})}\right)\left(\gamma \mathrm{U}_{1}+\left(\omega_{1}+\omega_{2}+\omega_{3}\right) \mathrm{U}_{1} \mathrm{P}_{1}-\gamma \mathrm{U}(\mathrm{t})\right)+\hbar\left(\omega_{1}+\omega_{2}+\omega_{3}\right) \mathrm{U}_{1} \mathrm{P}(\mathrm{t})
$$




$$
\begin{aligned}
& -v M_{1} e^{-\theta_{4} \tau_{4}} L_{1} \frac{L_{1} U\left(t-\tau_{1}\right) P\left(t-\tau_{1}\right)}{L(t) U_{1} P_{1}}+v M_{1} e^{-\theta_{4} \tau_{4}} L_{1}-M_{1} \omega_{2} e^{-\theta_{2} \tau_{2}-\theta_{4} \tau_{4}} U_{1} P_{1} \frac{I_{1} U\left(t-\tau_{2}\right) P\left(t-\tau_{2}\right)}{I(t) U_{1} P_{1}} \\
& -v M_{1} e^{-\theta_{4} \tau_{4}} L_{1} \frac{I_{1} L(t)}{I(t) L_{1}}+M_{1} \omega_{2} e^{-\theta_{2} \tau_{2}-\theta_{4} \tau_{4}} U_{1} P_{1}+v M_{1} e^{-\theta_{4} \tau_{4}} L_{1}-\Lambda M_{2} e^{-\theta_{5} \tau_{5}} \mathrm{O}_{1} \frac{O_{1} U\left(t-\tau_{3}\right) P\left(t-\tau_{3}\right)}{O(t) U_{1} P_{1}} \\
& +\wedge M_{2} e^{-\theta_{5} \tau_{5}} \mathrm{O}_{1}-\left(M_{1} \omega_{2} e^{-\theta_{2} \tau_{2}-\theta_{4} \tau_{4}} \mathrm{U}_{1} \mathrm{P}_{1}+v M_{1} e^{-\theta_{4} \tau_{4}} L_{1}\right) \frac{\mathrm{P}_{1} \mathrm{I}\left(\mathrm{t}-\tau_{4}\right)}{\mathrm{P}(\mathrm{t}) \mathrm{I}_{1}}-\Lambda M_{2} e^{-\theta_{5} \tau_{5}} \mathrm{O}_{1} \frac{\mathrm{P}_{1} \mathrm{O}\left(\mathrm{t}-\tau_{5}\right)}{\mathrm{P}(\mathrm{t}) \mathrm{O}_{1}} \\
& -\xi\left(\mathrm{P}(\mathrm{t})-\mathrm{P}_{1}\right)-\rho\left(\mathrm{P}(\mathrm{t})-\mathrm{P}_{1}\right) \mathrm{C}(\mathrm{t})+\rho\left(\mathrm{P}(\mathrm{t})-\mathrm{P}_{1}\right) \mathrm{C}_{1}-\rho\left(\mathrm{P}(\mathrm{t})-\mathrm{P}_{1}\right) \mathrm{C}_{1} \\
& +\frac{\rho}{\varepsilon-\vartheta C_{1}}\left(C(t)-C_{1}\right)\left(\varepsilon P(t)-\mu C(t)-\vartheta P(t) C(t)-\varepsilon P_{1}+\mu C_{1}+\vartheta P_{1} C_{1}-\vartheta P(t) C_{1}+\vartheta P(t) C_{1}\right) \\
& +v M_{1} e^{-\theta_{4} \tau_{4}} L_{1} \ln \left(\frac{U\left(t-\tau_{1}\right) P\left(t-\tau_{1}\right)}{U(t) P(t)}\right)+M_{1} \omega_{2} e^{-\theta_{2} \tau_{2}-\theta_{4} \tau_{4}} U_{1} P_{1} \ln \left(\frac{U\left(t-\tau_{2}\right) P\left(t-\tau_{2}\right)}{U(t) P(t)}\right) \\
& +M_{2} \omega_{3} e^{-\theta_{3} \tau_{3}-\theta_{5} \tau_{5}} \mathrm{U}_{1} \mathrm{P}_{1} \ln \left(\frac{\mathrm{U}\left(\mathrm{t}-\tau_{3}\right) \mathrm{P}\left(\mathrm{t}-\tau_{3}\right)}{\mathrm{U}(\mathrm{t}) \mathrm{P}(\mathrm{t})}\right)+\Lambda \mathrm{M}_{2} e^{-\theta_{5} \tau_{5}} \mathrm{O}_{1} \ln \left(\frac{\mathrm{O}\left(\mathrm{t}-\tau_{5}\right)}{\mathrm{O}(\mathrm{t})}\right) \\
& +\left(M_{1} \omega_{2} e^{-\theta_{2} \tau_{2}-\theta_{4} \tau_{4}} \mathrm{U}_{1} P_{1}+v M_{1} e^{-\theta_{4} \tau_{4}} L_{1}\right) \ln \left(\frac{I\left(t-\tau_{4}\right)}{I(t)}\right) .
\end{aligned}
$$

Simplifying the result, we obtain

$$
\begin{aligned}
& \frac{d W_{1}}{d t}=-\gamma \hbar \frac{\left(\mathrm{u}(\mathrm{t})-\mathrm{U}_{1}\right)^{2}}{\mathrm{U}(\mathrm{t})}+v \mathrm{M}_{1} e^{-\theta_{4} \tau_{4}} \mathrm{~L}_{1}\left(1-\frac{\mathrm{U}_{1}}{\mathrm{u}(\mathrm{t})}\right)+\mathrm{M}_{1} \omega_{2} e^{-\theta_{2} \tau_{2}-\theta_{4} \tau_{4}} \mathrm{U}_{1} \mathrm{P}_{1}\left(1-\frac{\mathrm{U}_{1}}{\mathrm{u}(\mathrm{t})}\right) \\
& +\Lambda M_{2} e^{-\theta_{5} \tau_{5}} \mathrm{O}_{1}\left(1-\frac{\mathrm{U}_{1}}{\mathrm{u}(\mathrm{t})}\right)+\left({ } \mathrm{M}_{1} e^{-\theta_{4} \tau_{4}} \mathrm{~L}_{1}+\mathrm{M}_{1} \omega_{2} e^{-\theta_{2} \tau_{2}-\theta_{4} \tau_{4}} \mathrm{U}_{1} \mathrm{P}_{1}+\Lambda \mathrm{M}_{2} e^{-\theta_{5} \tau_{5}} \mathrm{O}_{1}\right) \frac{\mathrm{P}(\mathrm{t})}{\mathrm{P}_{1}} \\
& -v M_{1} e^{-\theta_{4} \tau_{4}} L_{1} \frac{L_{1} U\left(t-\tau_{1}\right) P\left(t-\tau_{1}\right)}{L(t) U_{1} P_{1}}+v M_{1} e^{-\theta_{4} \tau_{4}} L_{1}-M_{1} \omega_{2} e^{-\theta_{2} \tau_{2}-\theta_{4} \tau_{4}} U_{1} P_{1} \frac{I_{1} U\left(t-\tau_{2}\right) P\left(t-\tau_{2}\right)}{I(t) U_{1} P_{1}} \\
& -v M_{1} e^{-\theta_{4} \tau_{4}} L_{1} \frac{I_{1} L(t)}{I(t) L_{1}}+M_{1} \omega_{2} e^{-\theta_{2} \tau_{2}-\theta_{4} \tau_{4}} U_{1} P_{1}+v M_{1} e^{-\theta_{4} \tau_{4}} L_{1}-\Lambda M_{2} e^{-\theta_{5} \tau_{5}} O_{1} \frac{O_{1} U\left(t-\tau_{3}\right) P\left(t-\tau_{3}\right)}{O(t) U_{1} P_{1}} \\
& +\wedge M_{2} e^{-\theta_{5} \tau_{5}} \mathrm{O}_{1}-\left(M_{1} \omega_{2} e^{-\theta_{2} \tau_{2}-\theta_{4} \tau_{4}} \mathrm{U}_{1} \mathrm{P}_{1}+v M_{1} e^{-\theta_{4} \tau_{4}} L_{1}\right) \frac{\mathrm{P}_{1} \mathrm{I}\left(\mathrm{t}-\tau_{4}\right)}{\mathrm{P}(\mathrm{t}) \mathrm{I}_{1}}-\Lambda M_{2} e^{-\theta_{5} \tau_{5}} \mathrm{O}_{1} \frac{\mathrm{P}_{1} \mathrm{O}\left(\mathrm{t}-\tau_{5}\right)}{\mathrm{P}(\mathrm{t}) \mathrm{O}_{1}} \\
& -\left(\xi+\rho C_{1}\right)\left(P(t)-P_{1}\right)-\rho\left(P(t)-P_{1}\right)\left(C(t)-C_{1}\right)+\frac{\rho\left(\varepsilon-\vartheta C_{1}\right)}{\varepsilon-\vartheta C_{1}}\left(C(t)-C_{1}\right)\left(P(t)-P_{1}\right) \\
& -\frac{\rho(\mu+\vartheta P(t))}{\varepsilon-\vartheta C_{1}}\left(C(t)-C_{1}\right)^{2}+v M_{1} e^{-\theta_{4} \tau_{4}} L_{1} \ln \left(\frac{U\left(t-\tau_{1}\right) P\left(t-\tau_{1}\right)}{U(t) P(t)}\right) \\
& +M_{1} \omega_{2} e^{-\theta_{2} \tau_{2}-\theta_{4} \tau_{4}} U_{1} P_{1} \ln \left(\frac{U\left(t-\tau_{2}\right) P\left(t-\tau_{2}\right)}{U(t) P(t)}\right)+M_{2} \omega_{3} e^{-\theta_{3} \tau_{3}-\theta_{5} \tau_{5}} U_{1} P_{1} \ln \left(\frac{U\left(t-\tau_{3}\right) P\left(t-\tau_{3}\right)}{U(t) P(t)}\right) \\
& +\left(M_{1} \omega_{2} e^{-\theta_{2} \tau_{2}-\theta_{4} \tau_{4}} \mathrm{U}_{1} \mathrm{P}_{1}+v \mathrm{M}_{1} e^{-\theta_{4} \tau_{4}} \mathrm{~L}_{1}\right) \ln \left(\frac{\mathrm{I}\left(\mathrm{t}-\tau_{4}\right)}{\mathrm{I}(\mathrm{t})}\right)+\Lambda \mathrm{M}_{2} e^{-\theta_{5} \tau_{5}} \mathrm{O}_{1} \ln \left(\frac{\mathrm{O}\left(\mathrm{t}-\tau_{5}\right)}{\mathrm{O}(\mathrm{t})}\right) .
\end{aligned}
$$

We have

$$
\begin{aligned}
-\left(\xi+\rho C_{1}\right)\left(P(t)-P_{1}\right) & =-\frac{1}{P_{1}}\left(\beta M_{1} e^{-\theta_{4} \tau_{4}} I_{1}+\Lambda M_{2} e^{-\theta_{5} \tau_{5}} O_{1}\right)\left(P(t)-P_{1}\right) \\
& =\left(M_{1} \omega_{2} e^{-\theta_{2} \tau_{2}-\theta_{4} \tau_{4}} U_{1} P_{1}+\nu M_{1} e^{-\theta_{4} \tau_{4}} L_{1}+\Lambda M_{2} e^{-\theta_{5} \tau_{5}} O_{1}\right)\left(1-\frac{P(t)}{P_{1}}\right) .
\end{aligned}
$$

Then $\frac{d W_{1}}{d t}$ will be

$$
\begin{aligned}
\frac{d W_{1}}{d t}= & -\gamma \hbar \frac{\left(U(t)-U_{1}\right)^{2}}{U(t)}-\frac{\rho(\mu+\vartheta P(t))}{\varepsilon-\vartheta C_{1}}\left(C(t)-C_{1}\right)^{2} \\
& +v M_{1} e^{-\theta_{4} \tau_{4}} L_{1}\left[4-\frac{U_{1}}{U(t)}-\frac{L_{1} U\left(t-\tau_{1}\right) P\left(t-\tau_{1}\right)}{L(t) U_{1} P_{1}}-\frac{I_{1} L(t)}{I(t) L_{1}}-\frac{P_{1} I\left(t-\tau_{4}\right)}{P(t) I_{1}}\right] \\
& +M_{1} \omega_{2} e^{-\theta_{2} \tau_{2}-\theta_{4} \tau_{4}} U_{1} P_{1}\left[3-\frac{U_{1}}{U(t)}-\frac{I_{1} U\left(t-\tau_{2}\right) P\left(t-\tau_{2}\right)}{I(t) U_{1} P_{1}}-\frac{P_{1} I\left(t-\tau_{4}\right)}{P(t) I_{1}}\right]
\end{aligned}
$$




$$
\begin{aligned}
& +\wedge M_{2} e^{-\theta_{5} \tau_{5}} \mathrm{O}_{1}\left[3-\frac{\mathrm{U}_{1}}{\mathrm{U}(\mathrm{t})}-\frac{\mathrm{O}_{1} \mathrm{U}\left(\mathrm{t}-\tau_{3}\right) \mathrm{P}\left(\mathrm{t}-\tau_{3}\right)}{\mathrm{O}(\mathrm{t}) \mathrm{U}_{1} \mathrm{P}_{1}}-\frac{\mathrm{P}_{1} \mathrm{O}\left(\mathrm{t}-\tau_{5}\right)}{\mathrm{P}(\mathrm{t}) \mathrm{O}_{1}}\right] \\
& +\nu M_{1} e^{-\theta_{4} \tau_{4}} \mathrm{~L}_{1}\left[\ln \left(\frac{\mathrm{U}\left(\mathrm{t}-\tau_{1}\right) \mathrm{P}\left(\mathrm{t}-\tau_{1}\right)}{\mathrm{U}(\mathrm{t}) \mathrm{P}(\mathrm{t})}\right)+\ln \left(\frac{\mathrm{I}\left(\mathrm{t}-\tau_{4}\right)}{\mathrm{I}(\mathrm{t})}\right)\right] \\
& +\mathrm{M}_{1} \omega_{2} \mathrm{e}^{-\theta_{2} \tau_{2}-\theta_{4} \tau_{4}} \mathrm{U}_{1} \mathrm{P}_{1}\left[\ln \left(\frac{\mathrm{U}\left(\mathrm{t}-\tau_{2}\right) \mathrm{P}\left(\mathrm{t}-\tau_{2}\right)}{\mathrm{U}(\mathrm{t}) \mathrm{P}(\mathrm{t})}\right)+\ln \left(\frac{\mathrm{I}\left(\mathrm{t}-\tau_{4}\right)}{\mathrm{I}(\mathrm{t})}\right)\right] \\
& +\wedge M_{2} e^{-\theta_{5} \tau_{5}} \mathrm{O}_{1}\left[\ln \left(\frac{\mathrm{U}\left(\mathrm{t}-\tau_{3}\right) \mathrm{P}\left(\mathrm{t}-\tau_{3}\right)}{\mathrm{U}(\mathrm{t}) \mathrm{P}(\mathrm{t})}\right)+\ln \left(\frac{\mathrm{O}\left(\mathrm{t}-\tau_{5}\right)}{\mathrm{O}(\mathrm{t})}\right)\right]
\end{aligned}
$$

Note that

$$
\begin{aligned}
\ln \left(\frac{\mathrm{U}\left(\mathrm{t}-\tau_{1}\right) \mathrm{P}\left(\mathrm{t}-\tau_{1}\right)}{\mathrm{U}(\mathrm{t}) \mathrm{P}(\mathrm{t})}\right) & =\ln \left(\frac{\mathrm{L}_{1} \mathrm{U}\left(\mathrm{t}-\tau_{1}\right) \mathrm{P}\left(\mathrm{t}-\tau_{1}\right)}{\mathrm{L}(\mathrm{t}) \mathrm{U}_{1} \mathrm{P}_{1}}\right)+\ln \left(\frac{\mathrm{I}_{1} \mathrm{~L}(\mathrm{t})}{\mathrm{I}(\mathrm{t}) \mathrm{L}_{1}}\right)+\ln \left(\frac{\mathrm{I}(\mathrm{t}) \mathrm{P}_{1}}{\mathrm{I}_{1} \mathrm{P}(\mathrm{t})}\right)+\ln \left(\frac{\mathrm{U}_{1}}{\mathrm{U}(\mathrm{t})}\right), \\
\ln \left(\frac{\mathrm{U}\left(\mathrm{t}-\tau_{2}\right) \mathrm{P}\left(\mathrm{t}-\tau_{2}\right)}{\mathrm{U}(\mathrm{t}) \mathrm{P}(\mathrm{t})}\right) & =\ln \left(\frac{\mathrm{I}_{1} \mathrm{U}\left(\mathrm{t}-\tau_{2}\right) \mathrm{P}\left(\mathrm{t}-\tau_{2}\right)}{\mathrm{I}(\mathrm{t}) \mathrm{U}_{1} \mathrm{P}_{1}}\right)+\ln \left(\frac{\mathrm{I}(\mathrm{t}) \mathrm{P}_{1}}{\mathrm{I}_{1} \mathrm{P}(\mathrm{t})}\right)+\ln \left(\frac{\mathrm{U}_{1}}{\mathrm{U}(\mathrm{t})}\right), \\
\ln \left(\frac{\mathrm{U}\left(\mathrm{t}-\tau_{3}\right) \mathrm{P}\left(\mathrm{t}-\tau_{3}\right)}{\mathrm{U}(\mathrm{t}) \mathrm{P}(\mathrm{t})}\right) & =\ln \left(\frac{\mathrm{O}_{1} \mathrm{U}\left(\mathrm{t}-\tau_{3}\right) \mathrm{P}\left(\mathrm{t}-\tau_{3}\right)}{\mathrm{O}(\mathrm{t}) \mathrm{U}_{1} \mathrm{P}_{1}}\right)+\ln \left(\frac{\mathrm{O}(\mathrm{t}) \mathrm{P}_{1}}{\mathrm{O}_{1} \mathrm{P}(\mathrm{t})}\right)+\ln \left(\frac{\mathrm{U}_{1}}{\mathrm{U}(\mathrm{t})}\right), \\
\ln \left(\frac{\mathrm{I}\left(\mathrm{t}-\tau_{4}\right)}{\mathrm{I}(\mathrm{t})}\right) & =\ln \left(\frac{\mathrm{P}_{1} \mathrm{I}\left(\mathrm{t}-\tau_{4}\right)}{\mathrm{P}(\mathrm{t}) \mathrm{I}_{1}}\right)+\ln \left(\frac{\mathrm{P}(\mathrm{t}) \mathrm{I}_{1}}{\mathrm{P}_{1} \mathrm{I}(\mathrm{t})}\right), \\
\ln \left(\frac{\mathrm{O}\left(\mathrm{t}-\tau_{5}\right)}{\mathrm{O}(\mathrm{t})}\right) & =\ln \left(\frac{\mathrm{P}_{1} \mathrm{O}\left(\mathrm{t}-\tau_{5}\right)}{\mathrm{P}(\mathrm{t}) \mathrm{O}_{1}}\right)+\ln \left(\frac{\mathrm{P}(\mathrm{t}) \mathrm{O}_{1}}{\mathrm{P}_{1} \mathrm{O}(\mathrm{t})}\right) .
\end{aligned}
$$

Then $\frac{d W_{1}}{d t}$ will be

$$
\begin{aligned}
\frac{d W_{1}}{d t}= & -\gamma \hbar \frac{\left(U(t)-U_{1}\right)^{2}}{U(t)}-\frac{\rho(\mu+\vartheta P(t))}{\varepsilon-\vartheta C_{1}}\left(C(t)-C_{1}\right)^{2} \\
& -v M_{1} e^{-\theta_{4} \tau_{4}} L_{1}\left[G\left(\frac{U_{1}}{U(t)}\right)+G\left(\frac{L_{1} U\left(t-\tau_{1}\right) P\left(t-\tau_{1}\right)}{L(t) U_{1} P_{1}}\right)+G\left(\frac{I_{1} L(t)}{I(t) L_{1}}\right)+G\left(\frac{P_{1} I\left(t-\tau_{4}\right)}{P(t) I_{1}}\right)\right] \\
& -M_{1} \omega_{2} e^{-\theta_{2} \tau_{2}-\theta_{4} \tau_{4}} U_{1} P_{1}\left[G\left(\frac{U_{1}}{U(t)}\right)+G\left(\frac{I_{1} U\left(t-\tau_{2}\right) P\left(t-\tau_{2}\right)}{I(t) U_{1} P_{1}}\right)+G\left(\frac{P_{1} I\left(t-\tau_{4}\right)}{P(t) I_{1}}\right)\right] \\
& -\Lambda M_{2} e^{-\theta_{5} \tau_{5}} O_{1}\left[G\left(\frac{U_{1}}{U(t)}\right)+G\left(\frac{O_{1} U\left(t-\tau_{3}\right) P\left(t-\tau_{3}\right)}{O(t) U_{1} P_{1}}\right)+G\left(\frac{P_{1} O\left(t-\tau_{5}\right)}{P(t) O_{1}}\right)\right] .
\end{aligned}
$$

Then for all $\mathrm{U}(\mathrm{t}), \mathrm{L}(\mathrm{t}), \mathrm{I}(\mathrm{t}), \mathrm{O}(\mathrm{t}), \mathrm{P}(\mathrm{t}), \mathrm{C}(\mathrm{t})>0$ we have $\frac{d W_{1}}{d t} \leqslant 0$. In addition, $\frac{d W_{1}}{d t}=0$ when $\mathrm{U}(\mathrm{t})=\mathrm{U}_{1}$, $\mathrm{L}(\mathrm{t})=\mathrm{L}_{1}, \mathrm{I}(\mathrm{t})=\mathrm{I}_{1}, \mathrm{O}(\mathrm{t})=\mathrm{O}_{1}, \mathrm{P}(\mathrm{t})=\mathrm{P}_{1}$, and $\mathrm{C}(\mathrm{t})=\mathrm{C}_{1}$. Let $\mathrm{D}_{1}=\{(\mathrm{U}(\mathrm{t}), \mathrm{L}(\mathrm{t}), \mathrm{I}(\mathrm{t}), \mathrm{O}(\mathrm{t}), \mathrm{P}(\mathrm{t}), \mathrm{C}(\mathrm{t}))$ : $\left.\frac{d W_{1}}{d t}=0\right\}$ and $N_{1}$ be the largest invariance subset of $D_{1}$. Clearly $N_{1}=\left\{E P_{1}\right\}$. Applying L.I.P we obtain that if $R_{0}>1$, then $E P_{1}$ is G.A.S.

\section{Model with general infection rate}

We generalize the model presented in the previous section by considering a more general incidence rate function $\Theta(U(t), P(t))$ as:

$$
\begin{aligned}
\dot{\mathrm{U}}(\mathrm{t}) & =\rho-\gamma \mathrm{U}(\mathrm{t})-\left(\omega_{1}+\omega_{2}+\omega_{3}\right) \Theta(\mathrm{U}(\mathrm{t}), \mathrm{P}(\mathrm{t})), \\
\dot{\mathrm{L}}(\mathrm{t}) & =e^{-\theta_{1} \tau_{1}} \omega_{1} \Theta\left(\mathrm{U}\left(\mathrm{t}-\tau_{1}\right), \mathrm{P}\left(\mathrm{t}-\tau_{1}\right)\right)-(\zeta+v) \mathrm{L}(\mathrm{t}), \\
\dot{\mathrm{I}}(\mathrm{t}) & =e^{-\theta_{2} \tau_{2}} \omega_{2} \Theta\left(\mathrm{U}\left(\mathrm{t}-\tau_{2}\right), \mathrm{P}\left(\mathrm{t}-\tau_{2}\right)\right)+v \mathrm{~L}(\mathrm{t})-\beta \mathrm{I}(\mathrm{t}), \\
\dot{\mathrm{O}}(\mathrm{t}) & =e^{-\theta_{3} \tau_{3}} \omega_{3} \Theta\left(\mathrm{U}\left(\mathrm{t}-\tau_{3}\right), \mathrm{P}\left(\mathrm{t}-\tau_{3}\right)\right)-\Lambda \mathrm{O}(\mathrm{t}),
\end{aligned}
$$




$$
\begin{aligned}
& \dot{\mathrm{P}}(\mathrm{t})=e^{-\theta_{4} \tau_{4}} \beta M_{1} \mathrm{I}\left(\mathrm{t}-\tau_{4}\right)+e^{-\theta_{5} \tau_{5}} \wedge M_{2} \mathrm{O}\left(\mathrm{t}-\tau_{5}\right)-\xi \mathrm{P}(\mathrm{t})-\rho \mathrm{P}(\mathrm{t}) \mathrm{C}(\mathrm{t}), \\
& \dot{\mathrm{C}}(\mathrm{t})=\varepsilon \mathrm{P}(\mathrm{t})-\mu \mathrm{C}(\mathrm{t})-\vartheta \mathrm{P}(\mathrm{t}) \mathrm{C}(\mathrm{t}) .
\end{aligned}
$$

We need the following assumptions on the function $\Theta(U, P)[18,33,49]$.

(A1) $\Theta(U, P)$ is continuously differentiable, $\Theta(U, P)>0$, and $\Theta(0, P)=\Theta(U, 0)=0$ for all $U>0$ and $P>0$.

(A2) $\frac{\partial \Theta(U, P)}{\partial U}>0, \frac{\partial \Theta(U, P)}{\partial P}>0$, and $\frac{\partial \Theta(U, 0)}{\partial P}>0$ for all $U>0$ and $P>0$.

(A3) $\frac{d}{d U}\left(\frac{\partial \Theta(U, 0)}{\partial P}\right)>0$ for all $U>0$.

(A4) $\frac{\Theta(U, P)}{P}$ is decreasing with respect to $P$ for all $P>0$.

This form of the incident rate $\Theta(U, P)$ with the above-mentioned Assumptions generalizes many popular forms like: bilinear incidence UP, saturated incidence UP/(1+ $\left.\alpha_{1} P\right)$, Beddington-DeAngelis incidence $\mathrm{UP} /\left(1+\alpha_{1} \mathrm{P}+\alpha_{2} \mathrm{U}\right)$, and Crowley-Martin incidence UP/((1+ $\left.\left.\alpha_{1} \mathrm{P}\right)\left(1+\alpha_{2} \mathrm{U}\right)\right)$, where, $\alpha_{1}$ and $\alpha_{2}$ are nonnegative constants.

One can show that $\mathrm{U}(\mathrm{t}), \mathrm{L}(\mathrm{t}), \mathrm{I}(\mathrm{t}), \mathrm{O}(\mathrm{t}), \mathrm{P}(\mathrm{t})$ and $\mathrm{C}(\mathrm{t})$ are all nonnegative and ultimately bounded under initial conditions (2.7).

Lemma 3.1. Assume that Assumptions (A1)-(A4) are satisfied, then there exists a threshold parameter $\mathrm{R}_{0}^{\mathrm{G}}>0$ such that:

(i) if $\mathrm{R}_{0}^{\mathrm{G}} \leqslant 1$, then the model has only one equilibrium point $\mathrm{EP}_{0}^{\mathrm{G}}$; and

(ii) if $\mathrm{R}_{0}^{\mathrm{G}}>1$, then the model has two equilibria $\mathrm{EP}_{0}^{\mathrm{G}}$ and $\mathrm{EP}_{1}^{\mathrm{G}}$.

Proof. At any equilibrium $\mathrm{EP}^{\mathrm{G}}(\mathrm{U}, \mathrm{L}, \mathrm{I}, \mathrm{O}, \mathrm{P}, \mathrm{C})$ we have

$$
\begin{aligned}
& \rho-\gamma \mathrm{U}-\left(\omega_{1}+\omega_{2}+\omega_{3}\right) \Theta(\mathrm{U}, \mathrm{P})=0, \\
& e^{-\theta_{1} \tau_{1}} \omega_{1} \Theta(\mathrm{U}, \mathrm{P})-(\zeta+v) \mathrm{L}=0, \\
& e^{-\theta_{2} \tau_{2}} \omega_{2} \Theta(\mathrm{U}, \mathrm{P})+v \mathrm{~L}-\beta \mathrm{I}=0,=0, \\
& e^{-\theta_{3} \tau_{3}} \omega_{3} \Theta(\mathrm{U}, \mathrm{P})-\wedge \mathrm{O}=0, \\
& e^{-\theta_{4} \tau_{4}} \beta M_{1} \mathrm{I}+e^{-\theta_{5} \tau_{5}} \wedge M_{2} \mathrm{O}-\xi \mathrm{P}-\rho \mathrm{PC}=0, \\
& \mathcal{P}-\mu \mathrm{C}-\vartheta \mathrm{PC}=0 .
\end{aligned}
$$

From Eq. (3.12), we have

$$
C=\frac{\varepsilon P}{\mu+\vartheta P^{\prime}}
$$

and from Eqs. (3.8) and (3.10), we obtain

$$
\mathrm{L}=\frac{e^{-\theta_{1} \tau_{1}+\theta_{3} \tau_{3}} \wedge \omega_{1}}{\omega_{3}(\zeta+v)} \mathrm{O}
$$

Now, from Eqs. (3.9)-(3.10) and (3.14), we get

$$
I=\frac{e^{-\theta_{2} \tau_{2}+\theta_{3} \tau_{3}} \wedge \omega_{2}(\zeta+\nu)+e^{-\theta_{1} \tau_{1}+\theta_{3} \tau_{3}} \wedge \omega_{1} \nu}{\beta \omega_{3}(\zeta+\nu)} O,
$$

and from Eqs. (3.11), (3.13), and (3.15), we have

$$
\mathrm{O}=\frac{\omega_{3}(\zeta+\nu)}{\Lambda \phi}\left(\xi \mathrm{P}+\frac{\rho \varepsilon \mathrm{P}^{2}}{\mu+\vartheta \mathrm{P}}\right)
$$

where $\phi=\left(e^{-\theta_{2} \tau_{2}+\theta_{3} \tau_{3}-\theta_{4} \tau_{4}} \omega_{2} M_{1}+e^{-\theta_{5} \tau_{5}} \omega_{3} M_{2}\right)(\zeta+v)+e^{-\theta_{1} \tau_{1}+\theta_{3} \tau_{3}-\theta_{4} \tau_{4}} \omega_{1} M_{1} v$. By substitution into 
Eqs. (3.14)-(3.15), we obtain

$$
\begin{aligned}
& L=\frac{e^{-\theta_{1} \tau_{1}+\theta_{3} \tau_{3}} \omega_{1}}{\phi}\left(\xi P+\frac{\rho \varepsilon P^{2}}{\mu+\vartheta P}\right), \\
& I=\frac{e^{-\theta_{2} \tau_{2}+\theta_{3} \tau_{3}} \omega_{2}(\zeta+\nu)+e^{-\theta_{1} \tau_{1}+\theta_{3} \tau_{3}} \omega_{1} \nu}{\beta \phi}\left(\xi P+\frac{\rho \varepsilon P^{2}}{\mu+\vartheta P}\right) .
\end{aligned}
$$

Then, from Eqs. (3.7), (3.10), and (3.16), we get

$$
\mathrm{U}=\frac{\rho}{\gamma}-\frac{e^{\Theta_{3} \tau_{3}}\left(\omega_{1}+\omega_{2}+\omega_{3}\right)(\zeta+\nu)}{\gamma \phi}\left(\xi \mathrm{P}+\frac{\rho \varepsilon \mathrm{P}^{2}}{\mu+\vartheta \mathrm{P}}\right)
$$

Let

$$
\Psi(\mathrm{P})=\frac{\rho}{\gamma}-\frac{e^{\theta_{3} \tau_{3}}\left(\omega_{1}+\omega_{2}+\omega_{3}\right)(\zeta+v)}{\gamma \phi}\left(\xi \mathrm{P}+\frac{\rho \varepsilon \mathrm{P}^{2}}{\mu+\vartheta \mathrm{P}}\right) .
$$

Therefore, we can write $\mathrm{U}$ as $\mathrm{U}=\Psi(\mathrm{P})$. Note that $\Psi(0)=\frac{\rho}{\gamma}$. From Eqs. (3.10) and (3.16), we have

$$
e^{-\theta_{3} \tau_{3}} \Theta(\Psi(P), P)-\frac{\zeta+\nu}{\varphi}\left(\xi P+\frac{\rho \varepsilon P^{2}}{\mu+\vartheta P}\right)=0 .
$$

Observe that, $\mathrm{P}=0$ is a solution of Eq. (3.20). Then from Eqs. (3.13), (3.16)-(3.19) we have $\mathrm{U}=\mathrm{U}_{0}=\frac{\rho}{\gamma}$, $\mathrm{L}=0, \mathrm{I}=0, \mathrm{O}=0$, and $\mathrm{C}=0$. Then we get an infection-free equilibrium $E P_{0}^{G}=\left(\mathrm{U}_{0}, 0,0,0,0,0\right)$. Let

$$
H(P)=e^{-\theta_{3} \tau_{3}} \Theta(\Psi(P), P)-\frac{\zeta+\nu}{\phi}\left(\xi P+\frac{\rho \varepsilon P^{2}}{\mu+\vartheta P}\right)
$$

then $\mathrm{H}(0)=0$. Let $\overline{\mathrm{P}}$ be such that $\Psi(\overline{\mathrm{P}})=0$, i.e.,

$$
\mathrm{U}_{0}-\frac{e^{\theta_{3} \tau_{3}}\left(\omega_{1}+\omega_{2}+\omega_{3}\right)(\zeta+v)}{\gamma \phi}\left(\xi \overline{\mathrm{P}}+\frac{\rho \varepsilon \overline{\mathrm{P}}^{2}}{\mu+\vartheta \overline{\mathrm{P}}}\right)=0,
$$

which gives

$$
e^{\theta_{3} \tau_{3}}(\rho \varepsilon+\xi \vartheta)(\zeta+v)\left(\omega_{1}+\omega_{2}+\omega_{3}\right) \bar{P}^{2}+\left[e^{\theta_{3} \tau_{3}} \xi \mu(\zeta+v)\left(\omega_{1}+\omega_{2}+\omega_{3}\right)-\gamma \vartheta U_{0} \phi\right] \bar{P}-\gamma \mu U_{0} \phi=0 .
$$

Thus, the positive solution of Eq. (3.21) is given by

$$
\overline{\mathrm{P}}=\frac{-\overline{\mathrm{b}}+\sqrt{\overline{\mathrm{b}}^{2}-4 \overline{\mathrm{ac}}}}{2 \overline{\mathrm{a}}}
$$

where,

$$
\begin{aligned}
& \bar{a}=e^{\theta_{3} \tau_{3}}(\rho \varepsilon+\xi \vartheta)(\zeta+v)\left(\omega_{1}+\omega_{2}+\omega_{3}\right), \\
& \bar{b}=e^{\theta_{3} \tau_{3}} \xi \mu(\zeta+v)\left(\omega_{1}+\omega_{2}+\omega_{3}\right)-\gamma \vartheta \mathrm{U}_{0} \phi, \\
& \bar{c}=-\gamma \mu \mathrm{U}_{0} \phi .
\end{aligned}
$$

We can see from Assumption (A1) that

$$
H(\bar{P})=e^{-\theta_{3} \tau_{3}} \Theta(0, \bar{P})-\frac{\zeta+\vartheta}{\phi}\left(\xi \bar{P}+\frac{\rho \varepsilon \bar{P}^{2}}{\mu+\vartheta \bar{P}}\right)=-\frac{\zeta+\vartheta}{\phi}\left(\xi \bar{P}+\frac{\rho \varepsilon \bar{P}^{2}}{\mu+\vartheta \bar{P}}\right)<0 .
$$


Moreover,

$$
H^{\prime}(P)=e^{-\theta_{3} \tau_{3}} \Psi^{\prime}(P) \frac{\partial \Theta(U, P)}{\partial U}+e^{-\theta_{3} \tau_{3}} \frac{\partial \Theta(U, P)}{\partial P}-\frac{\zeta+v}{\phi}\left(\xi+\frac{\rho \varepsilon\left(2 \mu P+\vartheta P^{2}\right)}{(\mu+\vartheta P)^{2}}\right) .
$$

Assumption (A1) implies that $\frac{\partial \Theta\left(\mathrm{U}_{0}, 0\right)}{\partial \mathrm{U}}=0$, then

$$
H^{\prime}(0)=e^{-\theta_{3} \tau_{3}} \frac{\partial \Theta\left(\mathrm{U}_{0}, 0\right)}{\partial P}-\frac{\xi(\zeta+v)}{\phi}=\frac{\xi(\zeta+v)}{\phi}\left(\frac{e^{-\theta_{3} \tau_{3}} \phi}{\xi(\zeta+v)} \frac{\partial \Theta\left(\mathrm{U}_{0}, 0\right)}{\partial P}-1\right)
$$

Therefore, if $\frac{e^{-\theta_{3} \tau_{3}} \phi}{\xi(\zeta+v)} \frac{\partial \Theta\left(U_{0}, 0\right)}{\partial P}>1$, then $H^{\prime}(0)>0$ and $\exists P_{1} \in(0, \bar{P})$ such that $H\left(P_{1}\right)=0$. Let us define

$$
R_{0}^{G}=\frac{\left(\omega_{2} M_{1} e^{-\theta_{2} \tau_{2}-\theta_{4} \tau_{4}}+\omega_{3} M_{2} e^{-\theta_{3} \tau_{3}-\theta_{5} \tau_{5}}\right)(\zeta+v)+v \omega_{1} M_{1} e^{-\theta_{1} \tau_{1}-\theta_{4} \tau_{4}}}{\xi(\zeta+v)} \frac{\partial \Theta\left(U_{0}, 0\right)}{\partial P}
$$

which represents the basic infection reproduction number. Now, let $P=P_{1}$ in Eq. (3.7) and define $g(U)=\rho-\gamma U-\left(\omega_{1}+\omega_{2}+\omega_{1}\right) \Theta\left(U, P_{1}\right)=0$. Then from Assumption (A1) we have $g(0)=\rho>0$ and $g\left(U_{0}\right)=-\left(\omega_{1}+\omega_{2}+\omega_{3}\right) \Theta\left(U_{0}, P_{1}\right)<0$. Assumption (A2) implies that $g(U)$ is strictly decreasing function of $\mathrm{U}$, and then there exists a unique $\mathrm{U}_{1} \in\left(0, \mathrm{U}_{0}\right)$ such that $g\left(\mathrm{U}_{1}\right)=0$. Moreover, from Eqs. (3.13) and (3.16)-(3.18), we have

$$
\begin{aligned}
\mathrm{L}_{1} & =\frac{e^{-\theta_{1} \tau_{1}+\theta_{3} \tau_{3}} \omega_{1}}{\phi}\left(\xi \mathrm{P}_{1}+\frac{\rho \varepsilon \mathrm{P}_{1}^{2}}{\mu+\vartheta \mathrm{P}_{1}}\right)>0, \\
\mathrm{I}_{1} & =\frac{e^{-\theta_{2} \tau_{2}+\theta_{3} \tau_{3}} \omega_{2}(\zeta+\nu)+e^{-\theta_{1} \tau_{1}+\theta_{3} \tau_{3}} \omega_{1} \nu}{\beta \phi}\left(\xi \mathrm{P}_{1}+\frac{\rho \varepsilon \mathrm{P}_{1}^{2}}{\mu+\vartheta \mathrm{P}_{1}}\right)>0, \\
\mathrm{O}_{1} & =\frac{\omega_{3}(\zeta+v)}{\Lambda \phi}\left(\xi \mathrm{P}_{1}+\frac{\rho \varepsilon \mathrm{P}_{1}^{2}}{\mu+\vartheta \mathrm{P}_{1}}\right)>0, \\
\mathrm{C}_{1} & =\frac{\varepsilon \mathrm{P}_{1}}{\mu+\vartheta \mathrm{P}_{1}}>0 .
\end{aligned}
$$

Therefore, the endemic equilibrium $E P_{1}^{G}=\left(U_{1}, L_{1}, I_{1}, O_{1}, P_{1}, C_{1}\right)$ exists if $R_{0}^{G}>1$.

\subsection{Global stability of equilibria}

The global stability analysis of the two equilibria of model (3.1)-(3.6) will be investigated in this subsection.

Theorem 3.2. Let $\mathrm{R}_{0}^{\mathrm{G}}<1$, then the infection-free equilibrium $\mathrm{EP}_{0}^{\mathrm{G}}$ of model (3.1)-(3.6) is G.A.S.

Proof. Construct a Lyapunov function $\mathrm{Z}_{0}(\mathrm{U}(\mathrm{t}), \mathrm{L}(\mathrm{t}), \mathrm{I}(\mathrm{t}), \mathrm{O}(\mathrm{t}), \mathrm{P}(\mathrm{t}), \mathrm{C}(\mathrm{t}))$ as

$$
\begin{aligned}
Z_{0}= & \hbar\left(u(t)-U_{0}-\int_{U_{0}}^{u(t)} \lim _{P \rightarrow 0^{+}} \frac{\Theta\left(U_{0}, P\right)}{\Theta(\eta, P)} d \eta\right)+\frac{v M_{1} e^{-\theta_{4} \tau_{4}}}{\zeta+v} L(t)+M_{1} e^{-\theta_{4} \tau_{4}} I(t) \\
& +M_{2} e^{-\theta_{5} \tau_{5}} \mathrm{O}(t)+P(t)+\frac{\xi}{\varepsilon}\left(1-R_{0}^{G}\right) C(t)+\frac{v M_{1} \omega_{1} e^{-\theta_{1} \tau_{1}-\theta_{4} \tau_{4}}}{\zeta+v} \int_{t-\tau_{1}}^{t} U(s) P(s) d s \\
& +M_{1} \omega_{2} e^{-\theta_{2} \tau_{2}-\theta_{4} \tau_{4}} \int_{t-\tau_{2}}^{t} U(s) P(s) d s+M_{2} \omega_{3} e^{-\theta_{3} \tau_{3}-\theta_{5} \tau_{5}} \int_{t-\tau_{3}}^{t} U(s) P(s) d s \\
& +\beta M_{1} e^{-\theta_{4} \tau_{4}} \int_{t-\tau_{4}}^{t} I(s) d s+\Lambda M_{2} e^{-\theta_{5} \tau_{5}} \int_{t-\tau_{5}}^{t} O(s) d s .
\end{aligned}
$$


Calculating $\frac{\mathrm{dZ}}{\mathrm{dt}}$ as:

$$
\begin{aligned}
\frac{d Z_{0}}{d t}= & \hbar\left(1-\lim _{P \rightarrow 0^{+}} \frac{\Theta\left(U_{0}, P\right)}{\Theta(U(t), P)}\right)\left(\rho-\gamma U(t)-\left(\omega_{1}+\omega_{2}+\omega_{3}\right) \Theta(U(t), P(t))\right) \\
& +\frac{v M_{1} e^{-\theta_{4} \tau_{4}}}{\zeta+v}\left(e^{-\theta_{1} \tau_{1}} \omega_{1} \Theta\left(U\left(t-\tau_{1}\right), P\left(t-\tau_{1}\right)\right)-(\zeta+v) L(t)\right) \\
& +M_{1} e^{-\theta_{4} \tau_{4}}\left(e^{-\theta_{2} \tau_{2}} \omega_{2} \Theta\left(U\left(t-\tau_{2}\right), P\left(t-\tau_{2}\right)+v L(t)-\beta I(t)\right)\right. \\
& +M_{2} e^{-\theta_{5} \tau_{5}}\left(e^{-\theta_{3} \tau_{3}} \omega_{3} \Theta\left(U\left(t-\tau_{3}\right), P\left(t-\tau_{3}\right)\right)-\Lambda O(t)\right) \\
& +\beta M_{1} e^{-\theta_{4} \tau_{4}} \mathrm{I}\left(t-\tau_{4}\right)+\Lambda M_{2} e^{-\theta_{5} \tau_{5}} \mathrm{O}\left(t-\tau_{5}\right)-\xi P(t)-\rho P(t) C(t) \\
& +\frac{\xi}{\varepsilon}\left(1-R_{0}^{G}\right)(\varepsilon P(t)-\mu C(t)-\vartheta P(t) C(t)) \\
& +\frac{v M_{1} \omega_{1} e^{-\theta_{1} \tau_{1}-\theta_{4} \tau_{4}}}{\zeta+v}\left(U(t) P(t)-U\left(t-\tau_{1}\right) P\left(t-\tau_{1}\right)\right) \\
& +M_{1} \omega_{2} e^{-\theta_{2} \tau_{2}-\theta_{4} \tau_{4}}\left(U(t) P(t)-U\left(t-\tau_{2}\right) P\left(t-\tau_{2}\right)\right) \\
& +M_{2} \omega_{3} e^{-\theta_{3} \tau_{3}-\theta_{5} \tau_{5}}\left(U(t) P(t)-U\left(t-\tau_{3}\right) P\left(t-\tau_{3}\right)\right) \\
& +\beta M_{1} e^{-\theta_{4} \tau_{4}}\left(I(t)-I\left(t-\tau_{4}\right)\right)+\Lambda M_{2} e^{-\theta_{5} \tau_{5}}\left(O(t)-O\left(t-\tau_{5}\right)\right)
\end{aligned}
$$

Using L'Hospital's Rule and simplifying the result, we get

$$
\begin{aligned}
\frac{d Z_{0}}{d t}= & \hbar\left(1-\frac{\partial \Theta\left(U_{0}, 0\right) / \partial P}{\partial \Theta(U, 0) / \partial P}\right)(\rho-\gamma U(t))+\hbar\left(\omega_{1}+\omega_{2}+\omega_{3}\right) \Theta(U(t), P(t)) \frac{\partial \Theta\left(U_{0}, 0\right) / \partial P}{\partial \Theta(U, 0) / \partial P} \\
& -\left(\rho+\frac{\xi \vartheta}{\varepsilon}\left(1-R_{0}^{G}\right)\right) P(t) C(t)-\xi R_{0}^{G} P(t)-\frac{\xi \mu}{\varepsilon}\left(1-R_{0}\right) C(t) \\
= & \hbar \gamma U_{0}\left(1-\frac{U(t)}{U_{0}}\right)\left(1-\frac{\partial \Theta\left(U_{0}, 0\right) / \partial P}{\partial \Theta(U, 0) / \partial P}\right)-\left(\rho+\frac{\xi \vartheta}{\varepsilon}\left(1-R_{0}^{G}\right)\right) P(t) C(t) \\
& +\xi R_{0}^{G}\left(\frac{\hbar}{\xi R_{0}^{G}} \frac{\Theta(U(t), P(t))}{P(t)} \frac{\partial \Theta\left(U_{0}, 0\right) / \partial P}{\partial \Theta(U, 0) / \partial P}-1\right) P(t)-\frac{\xi \mu}{\varepsilon}\left(1-R_{0}^{G}\right) C(t),
\end{aligned}
$$

where,

$$
\bar{\hbar}=\hbar\left(\omega_{1}+\omega_{2}+\omega_{3}\right) .
$$

From the Assumption (A4), we have

$$
\frac{\Theta(U, P)}{P} \leqslant \lim _{P \rightarrow 0^{+}} \frac{\Theta(U, P)}{P}=\frac{\partial \Theta(U, 0)}{\partial P}
$$

for all $\mathrm{U}(\mathrm{t})>0$. Then

$$
\frac{\bar{\hbar}}{\xi R_{0}^{G}} \frac{\Theta(U, P)}{P} \frac{\partial \Theta\left(U_{0}, 0\right) / \partial P}{\partial \Theta(U, 0) / \partial P} \leqslant \frac{\bar{\hbar}}{\xi R_{0}^{G}} \frac{\partial \Theta\left(U_{0}, 0\right)}{\partial P}=1
$$

It implies that

$$
\frac{d Z_{0}}{d t} \leqslant \hbar \gamma \mathrm{U}_{0}\left(1-\frac{\mathrm{U}(\mathrm{t})}{\mathrm{U}_{0}}\right)\left(1-\frac{\partial \Theta\left(\mathrm{U}_{0}, 0\right) / \partial \mathrm{P}}{\partial \Theta(\mathrm{U}, 0) / \partial \mathrm{P}}\right)-\left(\rho+\frac{\xi \vartheta}{\varepsilon}\left(1-\mathrm{R}_{0}^{\mathrm{G}}\right)\right) \mathrm{P}(\mathrm{t}) \mathrm{C}(\mathrm{t})-\frac{\xi \mu}{\varepsilon}\left(1-\mathrm{R}_{0}^{\mathrm{G}}\right) \mathrm{C}(\mathrm{t}) .
$$

From (A2) we have

$$
\left(1-\frac{\mathrm{u}}{\mathrm{U}_{0}}\right)\left(1-\frac{\partial \Theta\left(\mathrm{U}_{0}, 0\right) / \partial \mathrm{P}}{\partial \Theta(\mathrm{U}, 0) / \partial \mathrm{P}}\right) \leqslant 0
$$

Therefore, if $R_{0}^{G}<1$, then $\frac{d Z_{0}}{d t} \leqslant 0$ for all $U(t), P(t), C(t)>0$. Similar to the proof of Theorem 2.3, one can show that $E P_{0}^{G}$ is G.A.S. 
Theorem 3.3. Let $\mathrm{R}_{0}^{\mathrm{G}}>1$, then the endemic equilibrium $\mathrm{EP}_{1}^{\mathrm{G}}$ of model (3.1)-(3.6) is G.A.S.

Proof. Define $\mathrm{Z}_{11}(\mathrm{U}(\mathrm{t}), \mathrm{L}(\mathrm{t}), \mathrm{I}(\mathrm{t}), \mathrm{O}(\mathrm{t}), \mathrm{P}(\mathrm{t}), \mathrm{C}(\mathrm{t}))$ as

$$
\begin{aligned}
Z_{11}= & \hbar\left(U(t)-U_{1}-\int_{U_{1}}^{U(t)} \frac{\Theta\left(U_{1}, P_{1}\right)}{\Theta\left(\eta, P_{1}\right)} d \eta\right)+\frac{v M_{1} e^{-\theta_{4} \tau_{4}}}{\zeta+v} L_{1} G\left(\frac{L(t)}{L_{1}}\right)+M_{1} e^{-\theta_{4} \tau_{4}} I_{1} G\left(\frac{I(t)}{I_{1}}\right) \\
& +M_{2} e^{-\theta_{5} \tau_{5}} O_{1} G\left(\frac{O(t)}{O_{1}}\right)+P_{1} G\left(\frac{P(t)}{P_{1}}\right)+\frac{\rho}{2\left(\varepsilon-\vartheta C_{1}\right)}\left(C(t)-C_{1}\right)^{2} .
\end{aligned}
$$

Calculating $\frac{d Z_{11}}{d t}$ as:

$$
\begin{aligned}
\frac{d Z_{11}}{d t}= & \hbar\left(1-\frac{\Theta\left(U_{1}, P_{1}\right)}{\Theta\left(U(t), P_{1}\right)}\right)\left(\rho-\gamma \mathrm{U}(t)-\left(\omega_{1}+\omega_{2}+\omega_{3}\right) \Theta(U(t), P(t))\right) \\
& +\frac{v M_{1} e^{-\theta_{4} \tau_{4}}}{\zeta+v}\left(1-\frac{L_{1}}{L(t)}\right)\left(\omega_{1} e^{-\theta_{1} \tau_{1}} \Theta\left(U\left(t-\tau_{1}\right), P\left(t-\tau_{1}\right)\right)-(\zeta+v) L(t)\right) \\
& +M_{1} e^{-\theta_{4} \tau_{4}}\left(1-\frac{I_{1}}{I(t)}\right)\left(\omega_{2} e^{-\theta_{2} \tau_{2}} \Theta\left(U\left(t-\tau_{2}\right), P\left(t-\tau_{2}\right)\right)+v L(t)-\beta I(t)\right) \\
& +M_{2} e^{-\theta_{5} \tau_{5}}\left(1-\frac{O_{1}}{O(t)}\right)\left(\omega_{3} e^{-\theta_{3} \tau_{3}} \Theta\left(U\left(t-\tau_{3}\right), P\left(t-\tau_{3}\right)\right)-\Lambda O(t)\right) \\
& +\left(1-\frac{P_{1}}{P(t)}\right)\left(\beta M_{1} e^{-\theta_{4} \tau_{4}} \mathrm{I}\left(t-\tau_{4}\right)+\Lambda M_{2} e^{-\theta_{5} \tau_{5}} \mathrm{O}\left(t-\tau_{5}\right)-\xi P(t)-\rho P(t) C(t)\right) \\
& +\frac{\rho}{\varepsilon-\vartheta C_{1}}\left(C(t)-C_{1}\right)(\varepsilon P(t)-\mu C(t)-\vartheta P(t) C(t)) .
\end{aligned}
$$

Define $\mathrm{Z}_{12}(\mathrm{U}(\mathrm{t}), \mathrm{L}(\mathrm{t}), \mathrm{I}(\mathrm{t}), \mathrm{O}(\mathrm{t}), \mathrm{P}(\mathrm{t}), \mathrm{C}(\mathrm{t}))$ as:

$$
\begin{aligned}
Z_{12}= & \frac{v M_{1} \omega_{1} e^{-\theta_{1} \tau_{1}-\theta_{4} \tau_{4}}}{\zeta+v} \Theta\left(U_{1}, P_{1}\right) \int_{t-\tau_{1}}^{t} G\left(\frac{\Theta(U(s), P(s))}{\Theta\left(U_{1}, P_{1}\right)}\right) d s \\
& +M_{1} \omega_{2} e^{-\theta_{2} \tau_{2}-\theta_{4} \tau_{4}} \Theta\left(U_{1}, P_{1}\right) \int_{t-\tau_{2}}^{t} G\left(\frac{\Theta(U(s), P(s))}{\Theta\left(U_{1}, P_{1}\right)}\right) d s \\
& +M_{2} \omega_{3} e^{-\theta_{3} \tau_{3}-\theta_{5} \tau_{5}} \Theta\left(U_{1}, P_{1}\right) \int_{t-\tau_{3}}^{t} G\left(\frac{\Theta(U(s), P(s))}{\Theta\left(U_{1}, P_{1}\right)}\right) d s
\end{aligned}
$$

We calculate $\frac{d Z_{12}}{d t}$

$$
\begin{aligned}
\frac{d Z_{12}}{d t}= & \frac{v M_{1} \omega_{1} e^{-\theta_{1} \tau_{1}-\theta_{4} \tau_{4}}}{\zeta+v} \Theta\left(U_{1}, P_{1}\right)\left(\frac{\Theta(U(t), P(t))}{\Theta\left(U_{1}, P_{1}\right)}-\frac{\Theta\left(U\left(t-\tau_{1}\right), P\left(t-\tau_{1}\right)\right)}{\Theta\left(U_{1}, P_{1}\right)}\right. \\
& \left.+\ln \left(\frac{\Theta\left(U\left(t-\tau_{1}\right), P\left(t-\tau_{1}\right)\right)}{\Theta(U(t), P(t))}\right)\right] \\
& +M_{1} \omega_{2} e^{-\theta_{2} \tau_{2}-\theta_{4} \tau_{4}} \Theta\left(U_{1}, P_{1}\right)\left[\frac{\Theta(U(t), P(t))}{\Theta\left(U_{1}, P_{1}\right)}-\frac{\Theta\left(U\left(t-\tau_{2}\right), P\left(t-\tau_{2}\right)\right)}{\Theta\left(U_{1}, P_{1}\right)}\right. \\
& \left.+\ln \left(\frac{\Theta\left(U\left(t-\tau_{2}\right), P\left(t-\tau_{2}\right)\right)}{\Theta(U(t), P(t))}\right)\right] \\
& +M_{2} \omega_{3} e^{-\theta_{3} \tau_{3}-\theta_{5} \tau_{5} \Theta\left(U_{1}, P_{1}\right)}\left[\frac{\Theta(U(t), P(t))}{\Theta\left(U_{1}, P_{1}\right)}-\frac{\Theta\left(U\left(t-\tau_{3}\right), P\left(t-\tau_{3}\right)\right)}{\Theta\left(U_{1}, P_{1}\right)}\right. \\
& \left.+\ln \left(\frac{\Theta\left(U\left(t-\tau_{3}\right), P\left(t-\tau_{3}\right)\right)}{\Theta(U(t), P(t))}\right)\right] .
\end{aligned}
$$


Define $\mathrm{Z}_{13}(\mathrm{U}(\mathrm{t}), \mathrm{L}(\mathrm{t}), \mathrm{I}(\mathrm{t}), \mathrm{O}(\mathrm{t}), \mathrm{P}(\mathrm{t}), \mathrm{C}(\mathrm{t}))$ as

$$
Z_{13}=\beta M_{1} e^{-\theta_{4} \tau_{4}} I_{1} \int_{t-\tau_{4}}^{t} G\left(\frac{I(s)}{I_{1}}\right) d s+\Lambda M_{2} e^{-\theta_{5} \tau_{5}} O_{1} \int_{t-\tau_{5}}^{t} G\left(\frac{O(s)}{O_{1}}\right) d s .
$$

Calculating $\frac{d Z_{13}}{d t}$ as:

$$
\begin{aligned}
\frac{d Z_{13}}{d t}= & \beta M_{1} e^{-\theta_{4} \tau_{4}} I_{1}\left[\frac{I(t)}{I_{1}}-\frac{I\left(t-\tau_{4}\right)}{I_{1}}+\ln \left(\frac{I\left(t-\tau_{4}\right)}{I(t)}\right)\right] \\
& +\Lambda M_{2} e^{-\theta_{5} \tau_{5}} O_{1}\left[\frac{O(t)}{O_{1}}-\frac{O\left(t-\tau_{5}\right)}{O_{1}}+\ln \left(\frac{O\left(t-\tau_{5}\right)}{O(t)}\right)\right] .
\end{aligned}
$$

Construct a Lyapunov function $\mathrm{Z}_{1}(\mathrm{U}(\mathrm{t}), \mathrm{L}(\mathrm{t}), \mathrm{I}(\mathrm{t}), \mathrm{O}(\mathrm{t}), \mathrm{P}(\mathrm{t}), \mathrm{C}(\mathrm{t}))$ as

$$
\mathrm{Z}_{1}=\mathrm{Z}_{11}+\mathrm{Z}_{12}+\mathrm{Z}_{13}
$$

It follows that

$$
\frac{d Z_{1}}{d t}=\frac{d Z_{11}}{d t}+\frac{d Z_{12}}{d t}+\frac{d Z_{13}}{d t}
$$

Now we have

$$
\begin{aligned}
\frac{d Z_{1}}{d t}= & \hbar\left(1-\frac{\Theta\left(U_{1}, P_{1}\right)}{\Theta\left(U(t), P_{1}\right)}\right)(\rho-\gamma U(t))+\hbar\left(\omega_{1}+\omega_{2}+\omega_{3}\right) \Theta\left(U_{1}, P_{1}\right) \frac{\Theta(U(t), P(t))}{\Theta\left(U(t), P_{1}\right)} \\
& -\frac{v M_{1} \omega_{1} e^{-\theta_{1} \tau_{1}-\theta_{4} \tau_{4}}}{\zeta+v} \Theta\left(U\left(t-\tau_{1}\right), P\left(t-\tau_{1}\right)\right) \frac{L_{1}}{L(t)}+v M_{1} e^{-\theta_{4} \tau_{4}} L_{1} \\
& -M_{1} \omega_{2} e^{-\theta_{2} \tau_{2}-\theta_{4} \tau_{4}} \Theta\left(U\left(t-\tau_{2}\right), P\left(t-\tau_{2}\right)\right) \frac{I_{1}}{I(t)}-v M_{1} e^{-\theta_{4} \tau_{4}} L(t) \frac{I_{1}}{I(t)}+\beta M_{1} e^{-\theta_{4} \tau_{4} I_{1}} \\
& -M_{2} \omega_{3} e^{-\theta_{3} \tau_{3}-\theta_{5} \tau_{5}} \Theta\left(U\left(t-\tau_{3}\right), P\left(t-\tau_{3}\right)\right) \frac{O_{1}}{O(t)}+\wedge M_{2} e^{-\theta_{4} \tau_{4}} O_{1} \\
& -\beta M_{1} e^{-\theta_{4} \tau_{4}} I\left(t-\tau_{4}\right) \frac{P_{1}}{P(t)}-\Lambda M_{2} e^{-\theta_{5} \tau_{5}} \mathrm{O}\left(t-\tau_{5}\right) \frac{P_{1}}{P(t)}-\xi\left(P(t)-P_{1}\right) \\
& -\rho\left(P(t)-P_{1}\right) C(t)+\frac{\rho}{\varepsilon-\vartheta C_{1}}\left(C(t)-C_{1}\right)(\varepsilon P(t)-\mu C(t)-\vartheta P(t) C(t)) \\
& +\frac{v M_{1} \omega_{1} e^{-\theta_{1} \tau_{1}-\theta_{4} \tau_{4}} \Theta\left(U_{1}, P_{1}\right) \ln \left(\frac{\Theta\left(U\left(t-\tau_{1}\right), P\left(t-\tau_{1}\right)\right)}{\Theta(U(t), P(t))}\right)}{\zeta+v} \\
& +M_{1} \omega_{2} e^{-\theta_{2} \tau_{2}-\theta_{4} \tau_{4}} \Theta\left(U_{1}, P_{1}\right) \ln \left(\frac{\Theta\left(U\left(t-\tau_{2}\right), P\left(t-\tau_{2}\right)\right)}{\Theta(U(t), P(t))}\right) \\
& +M_{2} \omega_{3} e^{-\theta_{3} \tau_{3}-\theta_{5} \tau_{5}} \Theta\left(U_{1}, P_{1}\right) \ln \left(\frac{\Theta\left(U\left(t-\tau_{3}\right), P\left(t-\tau_{3}\right)\right)}{\Theta(U(t), P(t))}\right) \\
& +\beta M_{1} e^{-\theta_{4} \tau_{4}} I_{1} \ln \left(\frac{I\left(t-\tau_{4}\right)}{I(t)}\right)+\Lambda M_{2} e^{-\theta_{5} \tau_{5}} O_{1} \ln \left(\frac{O\left(t-\tau_{5}\right)}{O(t)}\right) .
\end{aligned}
$$

From the equilibrium conditions, we have:

$$
\begin{aligned}
\rho & =\gamma \mathrm{U}_{1}+\left(\omega_{1}+\omega_{2}+\omega_{3}\right) \Theta\left(\mathrm{U}_{1}, \mathrm{P}_{1}\right), \\
\frac{v \mathrm{M}_{1} \omega_{1} e^{-\theta_{1} \tau_{1}-\theta_{4} \tau_{4}}}{\zeta+v} \Theta\left(\mathrm{U}_{1}, \mathrm{P}_{1}\right) & =v M_{1} e^{-\theta_{4} \tau_{4}} \mathrm{~L}_{1}, \\
\beta M_{1} e^{-\theta_{4} \tau_{4}} \mathrm{I}_{1} & =\mathrm{M}_{1} \omega_{2} e^{-\theta_{2} \tau_{2}-\theta_{4} \tau_{4}} \Theta\left(\mathrm{U}_{1}, \mathrm{P}_{1}\right)+v M_{1} e^{-\theta_{4} \tau_{4}} \mathrm{~L}_{1}, \\
\mathrm{M}_{2} \omega_{3} e^{-\theta_{3} \tau_{3}-\theta_{5} \tau_{5}} \Theta\left(\mathrm{U}_{1}, \mathrm{P}_{1}\right) & =\wedge M_{2} e^{-\theta_{5} \tau_{5}} \mathrm{O}_{1}
\end{aligned}
$$




$$
\begin{aligned}
\xi \mathrm{P}_{1}+\rho \mathrm{P}_{1} \mathrm{C}_{1} & =\beta \mathrm{M}_{1} e^{-\theta_{4} \tau_{4}} \mathrm{I}_{1}+\Lambda \mathrm{M}_{2} \mathrm{e}^{-\theta_{5} \tau_{5}} \mathrm{O}_{1} \\
\varepsilon \mathrm{P}_{1} & =\mu \mathrm{C}_{1}+\vartheta \mathrm{P}_{1} \mathrm{C}_{1} .
\end{aligned}
$$

Utilizing these conditions for $E P_{1}^{G}$, we obtain

$$
\begin{aligned}
& \frac{d Z_{1}}{d t}=\hbar\left(1-\frac{\Theta\left(\mathrm{U}_{1}, \mathrm{P}_{1}\right)}{\Theta\left(\mathrm{U}(\mathrm{t}), \mathrm{P}_{1}\right)}\right)\left(\gamma \mathrm{U}_{1}-\gamma \mathrm{U}(\mathrm{t})\right) \\
& +\left(v M_{1} e^{-\theta_{4} \tau_{4}} L_{1}+M_{1} \omega_{2} e^{-\theta_{2} \tau_{2}-\theta_{4} \tau_{4}} \Theta\left(\mathrm{U}_{1}, \mathrm{P}_{1}\right)+\Lambda M_{2} e^{-\theta_{5} \tau_{5}} \mathrm{O}_{1}\right)\left(1-\frac{\Theta\left(\mathrm{U}_{1}, \mathrm{P}_{1}\right)}{\Theta\left(\mathrm{U}(\mathrm{t}), \mathrm{P}_{1}\right)}\right) \\
& +\left(v M_{1} e^{-\theta_{4} \tau_{4}} L_{1}+M_{1} \omega_{2} e^{-\theta_{2} \tau_{2}-\theta_{4} \tau_{4}} \Theta\left(U_{1}, P_{1}\right)+\Lambda M_{2} e^{-\theta_{5} \tau_{5}} O_{1}\right) \frac{\Theta(U(t), P(t))}{\Theta\left(U(t), P_{1}\right)} \\
& -v M_{1} e^{-\theta_{4} \tau_{4}} \frac{L_{1} \Theta\left(U\left(t-\tau_{1}\right), P\left(t-\tau_{1}\right)\right)}{L(t) \Theta\left(U_{1}, P_{1}\right)}+v M_{1} e^{-\theta_{4} \tau_{4}} L_{1} \\
& -M_{1} \omega_{2} e^{-\theta_{2} \tau_{2}-\theta_{4} \tau_{4}} \Theta\left(\mathrm{U}_{1}, \mathrm{P}_{1}\right) \frac{\mathrm{I}_{1} \Theta\left(\mathrm{U}\left(\mathrm{t}-\tau_{2}\right), \mathrm{P}\left(\mathrm{t}-\tau_{2}\right)\right)}{\mathrm{I}(\mathrm{t}) \Theta\left(\mathrm{U}_{1}, \mathrm{P}_{1}\right)}-v M_{1} e^{-\theta_{4} \tau_{4}} \mathrm{~L}_{1} \frac{\mathrm{I}_{1} \mathrm{~L}(\mathrm{t})}{\mathrm{I}(\mathrm{t}) \mathrm{L}_{1}} \\
& +M_{1} \omega_{2} e^{-\theta_{2} \tau_{2}-\theta_{4} \tau_{4}} \Theta\left(U_{1}, P_{1}\right)+v M_{1} e^{-\theta_{4} \tau_{4}} L_{1}-\Lambda M_{2} e^{-\theta_{5} \tau_{5}} O_{1} \frac{O_{1} \Theta\left(U\left(t-\tau_{3}\right), P\left(t-\tau_{3}\right)\right)}{O(t) \Theta\left(U_{1}, P_{1}\right)} \\
& +\Lambda M_{2} e^{-\theta_{5} \tau_{5}} \mathrm{O}_{1}-\left(v M_{1} e^{-\theta_{4} \tau_{4}} L_{1}+M_{1} \omega_{2} e^{-\theta_{2} \tau_{2}-\theta_{4} \tau_{4}} \Theta\left(U_{1}, P_{1}\right)\right) \frac{P_{1} I\left(t-\tau_{4}\right)}{P(t) I_{1}} \\
& -\Lambda M_{2} e^{-\theta_{5} \tau_{5}} \mathrm{O}_{1} \frac{\mathrm{P}_{1} \mathrm{O}\left(\mathrm{t}-\tau_{5}\right)}{\mathrm{P}(\mathrm{t}) \mathrm{O}_{1}}-\xi\left(\mathrm{P}(\mathrm{t})-\mathrm{P}_{1}\right)-\rho\left(\mathrm{P}(\mathrm{t})-\mathrm{P}_{1}\right) \mathrm{C}(\mathrm{t})+\rho\left(\mathrm{P}(\mathrm{t})-\mathrm{P}_{1}\right) \mathrm{C}_{1}-\rho\left(\mathrm{P}(\mathrm{t})-\mathrm{P}_{1}\right) \mathrm{C}_{1} \\
& +\frac{\rho}{\varepsilon-\vartheta C_{1}}\left(C(t)-C_{1}\right)\left(\varepsilon P(t)-\mu C(t)-\vartheta P(t) C(t)-\varepsilon P_{1}+\mu C_{1}+\vartheta P_{1} C_{1}-\vartheta P(t) C_{1}+\vartheta P(t) C_{1}\right) \\
& +v M_{1} e^{-\theta_{4} \tau_{4}} L_{1} \ln \left(\frac{\Theta\left(U\left(t-\tau_{1}\right), P\left(t-\tau_{1}\right)\right)}{\Theta(U(t), P(t))}\right)+\wedge M_{2} e^{-\theta_{5} \tau_{5}} O_{1} \ln \left(\frac{\Theta\left(U\left(t-\tau_{3}\right), P\left(t-\tau_{3}\right)\right)}{\Theta(U(t), P(t))}\right) \\
& +M_{1} \omega_{2} e^{-\theta_{2} \tau_{2}-\theta_{4} \tau_{4}} \Theta\left(U_{1}, P_{1}\right) \ln \left(\frac{\Theta\left(U\left(t-\tau_{2}\right), P\left(t-\tau_{2}\right)\right)}{\Theta(U(t), P(t))}\right) \\
& +\left(v M_{1} e^{-\theta_{4} \tau_{4}} L_{1}+M_{1} \omega_{2} e^{-\theta_{2} \tau_{2}-\theta_{4} \tau_{4}} \Theta\left(U_{1}, P_{1}\right)\right) \ln \left(\frac{I\left(t-\tau_{4}\right)}{I(t)}\right)+\Lambda M_{2} e^{-\theta_{5} \tau_{5}} \mathrm{O}_{1} \ln \left(\frac{O\left(t-\tau_{5}\right)}{O(t)}\right) .
\end{aligned}
$$

Simplifying the result, we obtain

$$
\begin{aligned}
& \frac{d Z_{1}}{d t}=-\gamma \hbar U_{1}\left(1-\frac{U(t)}{U_{1}}\right)\left(1-\frac{\Theta\left(U_{1}, P_{1}\right)}{\Theta\left(U(t), P_{1}\right)}\right)-\left(\xi+\rho C_{1}\right)\left(P(t)-P_{1}\right) \\
& -\rho\left(P(t)-P_{1}\right)\left(C(t)-C_{1}\right)+\frac{\rho\left(\varepsilon-\vartheta C_{1}\right)}{\varepsilon-\vartheta C_{1}}\left(C(t)-C_{1}\right)\left(P(t)-P_{1}\right)-\frac{\rho(\mu+\vartheta P(t))}{\varepsilon-\vartheta C_{1}}\left(C(t)-C_{1}\right)^{2} \\
& +v M_{1} e^{-\theta_{4} \tau_{4}} L_{1}\left[3-\frac{\Theta\left(U_{1}, P_{1}\right)}{\Theta\left(U(t), P_{1}\right)}-\frac{L_{1} \Theta\left(U\left(t-\tau_{1}\right), P\left(t-\tau_{1}\right)\right)}{L(t) \Theta\left(U_{1}, P_{1}\right)}-\frac{I_{1} L(t)}{I(t) L_{1}}-\frac{P_{1} I\left(t-\tau_{4}\right)}{P(t) I_{1}}\right. \\
& \left.+\ln \left(\frac{\Theta\left(\mathrm{U}\left(\mathrm{t}-\tau_{1}\right), \mathrm{P}\left(\mathrm{t}-\tau_{1}\right)\right)}{\Theta(\mathrm{U}(\mathrm{t}), \mathrm{P}(\mathrm{t}))}\right)+\ln \left(\frac{\mathrm{I}\left(\mathrm{t}-\tau_{4}\right)}{\mathrm{I}(\mathrm{t})}\right)\right] \\
& +M_{1} \omega_{2} e^{-\theta_{2} \tau_{2}-\theta_{4} \tau_{4}} \Theta\left(U_{1}, P_{1}\right)\left[2-\frac{\Theta\left(U_{1}, P_{1}\right)}{\Theta\left(U(t), P_{1}\right)}-\frac{P_{1} I\left(t-\tau_{4}\right)}{P(t) I_{1}}-\frac{I_{1} \Theta\left(U\left(t-\tau_{2}\right), P\left(t-\tau_{2}\right)\right)}{I(t) \Theta\left(U_{1}, P_{1}\right)}\right. \\
& \left.+\ln \left(\frac{\Theta\left(\mathrm{U}\left(\mathrm{t}-\tau_{2}\right), \mathrm{P}\left(\mathrm{t}-\tau_{2}\right)\right)}{\Theta(\mathrm{U}(\mathrm{t}), \mathrm{P}(\mathrm{t}))}\right)+\ln \left(\frac{\mathrm{I}\left(\mathrm{t}-\tau_{4}\right)}{\mathrm{I}(\mathrm{t})}\right)\right] \\
& +\wedge M_{2} e^{-\theta_{5} \tau_{5}} \mathrm{O}_{1}\left[2-\frac{\Theta\left(\mathrm{U}_{1}, \mathrm{P}_{1}\right)}{\Theta\left(\mathrm{U}(\mathrm{t}), \mathrm{P}_{1}\right)}-\frac{\mathrm{O}_{1} \Theta\left(\mathrm{U}\left(\mathrm{t}-\tau_{3}\right), \mathrm{P}\left(\mathrm{t}-\tau_{3}\right)\right)}{\mathrm{O}(\mathrm{t}) \Theta\left(\mathrm{U}_{1}, \mathrm{P}_{1}\right)}-\frac{\mathrm{P}_{1} \mathrm{O}\left(\mathrm{t}-\tau_{5}\right)}{\mathrm{P}(\mathrm{t}) \mathrm{O}_{1}}\right. \\
& \left.+\ln \left(\frac{\Theta\left(\mathrm{U}\left(\mathrm{t}-\tau_{3}\right), \mathrm{P}\left(\mathrm{t}-\tau_{3}\right)\right)}{\Theta(\mathrm{U}(\mathrm{t}), \mathrm{P}(\mathrm{t}))}\right)+\ln \left(\frac{\mathrm{O}\left(\mathrm{t}-\tau_{5}\right)}{\mathrm{O}(\mathrm{t})}\right)\right]
\end{aligned}
$$




$$
\begin{aligned}
& +\left(v M_{1} e^{-\theta_{4} \tau_{4}} \mathrm{~L}_{1}+M_{1} \omega_{2} e^{-\theta_{2} \tau_{2}-\theta_{4} \tau_{4}} \Theta\left(\mathrm{U}_{1}, \mathrm{P}_{1}\right)+\Lambda M_{2} e^{-\theta_{5} \tau_{5}} \mathrm{O}_{1}\right) \frac{\Theta(\mathrm{U}(\mathrm{t}), \mathrm{P}(\mathrm{t}))}{\Theta\left(\mathrm{U}(\mathrm{t}), \mathrm{P}_{1}\right)} \\
& +\left(v \mathrm{M}_{1} e^{-\theta_{4} \tau_{4}} \mathrm{~L}_{1}+\mathrm{M}_{1} \omega_{2} e^{-\theta_{2} \tau_{2}-\theta_{4} \tau_{4}} \Theta\left(\mathrm{U}_{1}, \mathrm{P}_{1}\right)+\Lambda \mathrm{M}_{2} e^{-\theta_{5} \tau_{5}} \mathrm{O}_{1}\right)\left(1-\frac{\mathrm{P}(\mathrm{t}) \Theta\left(\mathrm{U}(\mathrm{t}), \mathrm{P}_{1}\right)}{\mathrm{P}_{1} \Theta(\mathrm{U}(\mathrm{t}), \mathrm{P}(\mathrm{t}))}\right) \\
& -\left(v \mathrm{M}_{1} e^{-\theta_{4} \tau_{4}} \mathrm{~L}_{1}+\mathrm{M}_{1} \omega_{2} e^{-\theta_{2} \tau_{2}-\theta_{4} \tau_{4}} \Theta\left(\mathrm{U}_{1}, \mathrm{P}_{1}\right)+\Lambda \mathrm{M}_{2} e^{-\theta_{5} \tau_{5}} \mathrm{O}_{1}\right)\left(1-\frac{\mathrm{P}(\mathrm{t}) \Theta\left(\mathrm{U}(\mathrm{t}), \mathrm{P}_{1}\right)}{\mathrm{P}_{1} \Theta(\mathrm{U}(\mathrm{t}), \mathrm{P}(\mathrm{t}))}\right)
\end{aligned}
$$

Note that

$$
\begin{aligned}
-\left(\xi+\rho C_{1}\right)\left(P(t)-P_{1}\right) & =-\frac{1}{P_{1}}\left(\beta M_{1} e^{-\theta_{4} \tau_{4}} I_{1}+\Lambda M_{2} e^{-\theta_{5} \tau_{5}} O_{1}\right)\left(P(t)-P_{1}\right) \\
& =\left(v M_{1} e^{-\theta_{4} \tau_{4}} L_{1}+M_{1} \omega_{2} e^{-\theta_{2} \tau_{2}-\theta_{4} \tau_{4}} \Theta\left(U_{1}, P_{1}\right)+e^{-\theta_{5} \tau_{5}} \wedge M_{2} O_{1}\right)\left(1-\frac{P(t)}{P_{1}}\right) .
\end{aligned}
$$

Moreover, we have

$$
\begin{aligned}
\ln \left(\frac{\Theta\left(U\left(t-\tau_{1}\right), P\left(t-\tau_{1}\right)\right)}{\Theta(U(t), P(t))}\right)= & \ln \left(\frac{L_{1} \Theta\left(U\left(t-\tau_{1}\right), P\left(t-\tau_{1}\right)\right)}{L(t) \Theta\left(U_{1}, P_{1}\right)}\right)+\ln \left(\frac{I_{1} L(t)}{I(t) L_{1}}\right) \\
& +\ln \left(\frac{I(t) P_{1}}{I_{1} P(t)}\right)+\ln \left(\frac{P(t) \Theta\left(U(t), P_{1}\right)}{P_{1} \Theta(U(t), P(t))}\right)+\ln \left(\frac{\Theta\left(U_{1}, P_{1}\right)}{\Theta\left(U(t), P_{1}\right)}\right), \\
\ln \left(\frac{\Theta\left(U\left(t-\tau_{2}\right), P\left(t-\tau_{2}\right)\right)}{\Theta(U(t), P(t))}\right)= & \ln \left(\frac{I_{1} \Theta\left(U\left(t-\tau_{2}\right), P\left(t-\tau_{2}\right)\right)}{I(t) \Theta\left(U_{1}, P_{1}\right)}\right)+\ln \left(\frac{I(t) P_{1}}{I_{1} P(t)}\right)+\ln \left(\frac{\Theta\left(U_{1}, P_{1}\right)}{\Theta\left(U(t), P_{1}\right)}\right) \\
& +\ln \left(\frac{P(t) \Theta\left(U(t), P_{1}\right)}{P_{1} \Theta(U(t), P(t))}\right), \\
\ln \left(\frac{\Theta\left(U\left(t-\tau_{3}\right), P\left(t-\tau_{3}\right)\right)}{\Theta(U(t), P(t))}\right)= & \ln \left(\frac{O_{1} \Theta\left(U\left(t-\tau_{3}\right), P\left(t-\tau_{3}\right)\right)}{O(t) \Theta\left(U_{1}, P_{1}\right)}\right)+\ln \left(\frac{O(t) P_{1}}{O_{1} P(t)}\right)+\ln \left(\frac{\Theta\left(U_{1}, P_{1}\right)}{\Theta\left(U(t), P_{1}\right)}\right) \\
& +\ln \left(\frac{P(t) \Theta\left(U(t), P_{1}\right)}{P_{1} \Theta(U(t), P(t))}\right), \\
\ln \left(\frac{I\left(t-\tau_{4}\right)}{I(t)}\right)= & \ln \left(\frac{P_{1} I\left(t-\tau_{4}\right)}{P(t) I_{1}}\right)+\ln \left(\frac{P(t) I_{1}}{P_{1} I(t)}\right), \\
\ln \left(\frac{O\left(t-\tau_{5}\right)}{O(t)}\right)= & \ln \left(\frac{P_{1} O\left(t-\tau_{5}\right)}{P(t) O_{1}}\right)+\ln \left(\frac{P(t) O_{1}}{P_{1} O(t)}\right) .
\end{aligned}
$$

Then $\frac{d Z_{1}}{d t}$ will be

$$
\begin{aligned}
& \frac{d Z_{1}}{d t}=-\gamma \hbar U_{1}\left(1-\frac{U(t)}{U_{1}}\right)\left(1-\frac{\Theta\left(U_{1}, P_{1}\right)}{\Theta\left(U(t), P_{1}\right)}\right)-\frac{\rho(\mu+\vartheta P(t))}{\varepsilon-\vartheta C_{1}}\left(C(t)-C_{1}\right)^{2} \\
& +v M_{1} e^{-\theta_{4} \tau_{4}} L_{1}\left[5-\frac{\Theta\left(\mathrm{U}_{1}, \mathrm{P}_{1}\right)}{\Theta\left(\mathrm{U}(\mathrm{t}), \mathrm{P}_{1}\right)}-\frac{\mathrm{L}_{1} \Theta\left(\mathrm{U}\left(\mathrm{t}-\tau_{1}\right), \mathrm{P}\left(\mathrm{t}-\tau_{1}\right)\right)}{\mathrm{L}(\mathrm{t}) \Theta\left(\mathrm{U}_{1}, \mathrm{P}_{1}\right)}-\frac{\mathrm{I}_{1} \mathrm{~L}(\mathrm{t})}{\mathrm{I}(\mathrm{t}) \mathrm{L}_{1}}\right. \\
& \left.-\frac{P_{1} I\left(t-\tau_{4}\right)}{P(t) I_{1}}-\frac{P(t) \Theta\left(U(t), P_{1}\right)}{P_{1} \Theta(U(t), P(t))}+\ln \left(\frac{\Theta\left(U\left(t-\tau_{1}\right), P\left(t-\tau_{1}\right)\right)}{\Theta(U(t), P(t))}\right)+\ln \left(\frac{I\left(t-\tau_{4}\right)}{I(t)}\right)\right] \\
& +M_{1} \omega_{2} e^{-\theta_{2} \tau_{2}-\theta_{4} \tau_{4}} \Theta\left(\mathrm{U}_{1}, \mathrm{P}_{1}\right)\left[4-\frac{\Theta\left(\mathrm{U}_{1}, \mathrm{P}_{1}\right)}{\Theta\left(\mathrm{U}(\mathrm{t}), \mathrm{P}_{1}\right)}-\frac{\mathrm{P}_{1} \mathrm{I}\left(\mathrm{t}-\tau_{4}\right)}{\mathrm{P}(\mathrm{t}) \mathrm{I}_{1}}-\frac{\mathrm{I}_{1} \Theta\left(\mathrm{U}\left(\mathrm{t}-\tau_{2}\right), \mathrm{P}\left(\mathrm{t}-\tau_{2}\right)\right)}{\mathrm{I}(\mathrm{t}) \Theta\left(\mathrm{U}_{1}, \mathrm{P}_{1}\right)}\right. \\
& \left.-\frac{\mathrm{P}(\mathrm{t}) \Theta\left(\mathrm{U}(\mathrm{t}), \mathrm{P}_{1}\right)}{\mathrm{P}_{1} \Theta(\mathrm{U}(\mathrm{t}), \mathrm{P}(\mathrm{t}))}+\ln \left(\frac{\Theta\left(\mathrm{U}\left(\mathrm{t}-\tau_{2}\right), \mathrm{P}\left(\mathrm{t}-\tau_{2}\right)\right)}{\Theta(\mathrm{U}(\mathrm{t}), \mathrm{P}(\mathrm{t}))}\right)+\ln \left(\frac{\mathrm{I}\left(\mathrm{t}-\tau_{4}\right)}{\mathrm{I}(\mathrm{t})}\right)\right] \\
& +\wedge M_{2} e^{-\theta_{5} \tau_{5}} \mathrm{O}_{1}\left[4-\frac{\Theta\left(\mathrm{U}_{1}, \mathrm{P}_{1}\right)}{\Theta\left(\mathrm{U}(\mathrm{t}), \mathrm{P}_{1}\right)}-\frac{\mathrm{O}_{1} \Theta\left(\mathrm{U}\left(\mathrm{t}-\tau_{3}\right), \mathrm{P}\left(\mathrm{t}-\tau_{3}\right)\right)}{\mathrm{O}(\mathrm{t}) \Theta\left(\mathrm{U}_{1}, \mathrm{P}_{1}\right)}\right. \\
& \left.-\frac{\mathrm{P}_{1} \mathrm{O}\left(\mathrm{t}-\tau_{5}\right)}{\mathrm{P}(\mathrm{t}) \mathrm{O}_{1}}-\frac{\mathrm{P}(\mathrm{t}) \Theta\left(\mathrm{U}(\mathrm{t}), \mathrm{P}_{1}\right)}{\mathrm{P}_{1} \Theta(\mathrm{U}(\mathrm{t}), \mathrm{P}(\mathrm{t}))}+\ln \left(\frac{\Theta\left(\mathrm{U}\left(\mathrm{t}-\tau_{3}\right), \mathrm{P}\left(\mathrm{t}-\tau_{3}\right)\right)}{\Theta(\mathrm{U}(\mathrm{t}), \mathrm{P}(\mathrm{t}))}\right)+\ln \left(\frac{\mathrm{O}\left(\mathrm{t}-\tau_{5}\right)}{\mathrm{O}(\mathrm{t})}\right)\right]
\end{aligned}
$$




$$
\begin{aligned}
& +\left(v M_{1} e^{-\theta_{4} \tau_{4}} L_{1}+M_{1} \omega_{2} e^{-\theta_{2} \tau_{2}-\theta_{4} \tau_{4}} \Theta\left(U_{1}, P_{1}\right)+e^{-\theta_{5} \tau_{5}} \wedge M_{2} \mathrm{O}_{1}\right) \\
& \times\left(\frac{\Theta(U(t), P(t))}{\Theta\left(U(t), P_{1}\right)}-\frac{P(t)}{P_{1}}-1+\frac{P(t) \Theta\left(U(t), P_{1}\right)}{P_{1} \Theta(U(t), P(t))}\right) .
\end{aligned}
$$

Simplifying the result, we get

$$
\begin{aligned}
& \frac{d Z_{1}}{d t}=-\gamma \hbar U_{1}\left(1-\frac{U(t)}{U_{1}}\right)\left(1-\frac{\Theta\left(U_{1}, P_{1}\right)}{\Theta\left(U(t), P_{1}\right)}\right)-\frac{\rho(\mu+\vartheta P(t))}{\varepsilon-\vartheta C_{1}}\left(C(t)-C_{1}\right)^{2} \\
& -v M_{1} e^{-\theta_{4} \tau_{4}} L_{1}\left[G\left(\frac{\Theta\left(U_{1}, P_{1}\right)}{\Theta\left(U(t), P_{1}\right)}\right)+G\left(\frac{L_{1} \Theta\left(U\left(t-\tau_{1}\right), P\left(t-\tau_{1}\right)\right)}{L(t) \Theta\left(U_{1}, P_{1}\right)}\right)+G\left(\frac{I_{1} L(t)}{I(t) L_{1}}\right)\right. \\
& \left.+G\left(\frac{P_{1} I\left(t-\tau_{4}\right)}{P(t) I_{1}}\right)+G\left(\frac{P(t) \Theta\left(U(t), P_{1}\right)}{P_{1} \Theta(U(t), P(t))}\right)\right] \\
& -M_{1} \omega_{2} e^{-\theta_{2} \tau_{2}-\theta_{4} \tau_{4}} \Theta\left(U_{1}, P_{1}\right)\left[G\left(\frac{\Theta\left(U_{1}, P_{1}\right)}{\Theta\left(U(t), P_{1}\right)}\right)+G\left(\frac{I_{1} \Theta\left(U\left(t-\tau_{2}\right), P\left(t-\tau_{2}\right)\right)}{I(t) \Theta\left(U_{1}, P_{1}\right)}\right)\right. \\
& \left.+\mathrm{G}\left(\frac{\mathrm{P}_{1} \mathrm{I}\left(\mathrm{t}-\tau_{4}\right)}{\mathrm{P}(\mathrm{t}) \mathrm{I}_{1}}\right)+\mathrm{G}\left(\frac{\mathrm{P}(\mathrm{t}) \Theta\left(\mathrm{U}(\mathrm{t}), \mathrm{P}_{1}\right)}{\mathrm{P}_{1} \Theta(\mathrm{U}(\mathrm{t}), \mathrm{P}(\mathrm{t}))}\right)\right] \\
& -\wedge M_{2} e^{-\theta_{5} \tau_{5}} u_{1}\left[G\left(\frac{\Theta\left(\mathrm{U}_{1}, \mathrm{P}_{1}\right)}{\Theta\left(\mathrm{U}(\mathrm{t}), \mathrm{P}_{1}\right)}\right)+\mathrm{G}\left(\frac{\mathrm{O}_{1} \Theta\left(\mathrm{U}\left(\mathrm{t}-\tau_{3}\right), \mathrm{P}\left(\mathrm{t}-\tau_{3}\right)\right)}{\mathrm{O}(\mathrm{t}) \Theta\left(\mathrm{U}_{1}, \mathrm{P}_{1}\right)}\right)+\mathrm{G}\left(\frac{\mathrm{P}_{1} \mathrm{O}\left(\mathrm{t}-\tau_{5}\right)}{\mathrm{P}(\mathrm{t}) \mathrm{O}_{1}}\right)\right. \\
& \left.+G\left(\frac{P(t) \Theta\left(U(t), P_{1}\right)}{P_{1} \Theta(U(t), P(t))}\right)\right]+\left(v M_{1} e^{-\theta_{4} \tau_{4}} L_{1}+M_{1} \omega_{2} e^{-\theta_{2} \tau_{2}-\theta_{4} \tau_{4}} \Theta\left(U_{1}, P_{1}\right)+e^{-\theta_{5} \tau_{5}} \wedge M_{2} O_{1}\right) \\
& \times\left(\frac{\Theta(\mathrm{U}(\mathrm{t}), \mathrm{P}(\mathrm{t}))}{\Theta\left(\mathrm{U}(\mathrm{t}), \mathrm{P}_{1}\right)}-\frac{\mathrm{P}(\mathrm{t})}{\mathrm{P}_{1}}\right)\left(1-\frac{\Theta\left(\mathrm{U}(\mathrm{t}), \mathrm{P}_{1}\right)}{\Theta(\mathrm{U}(\mathrm{t}), \mathrm{P}(\mathrm{t}))}\right) .
\end{aligned}
$$

From (A2) we have

$$
\left(1-\frac{\mathrm{U}(\mathrm{t})}{\mathrm{U}_{1}}\right)\left(1-\frac{\Theta\left(\mathrm{U}_{1}, \mathrm{P}_{1}\right)}{\Theta\left(\mathrm{U}(\mathrm{t}), \mathrm{P}_{1}\right)}\right) \leqslant 0 .
$$

In addition, from Assumptions (A1), (A2), and (A4), we have

$$
\left(\frac{\Theta(U(t), P(t))}{P(t)}-\frac{\Theta\left(U(t), P_{1}\right)}{P_{1}}\right)\left(\Theta(U(t), P(t))-\Theta\left(U(t), P_{1}\right) \leqslant 0\right.
$$

which gives

$$
\left(\frac{\Theta(\mathrm{U}(\mathrm{t}), \mathrm{P}(\mathrm{t}))}{\Theta\left(\mathrm{U}(\mathrm{t}), \mathrm{P}_{1}\right)}-\frac{\mathrm{P}(\mathrm{t})}{\mathrm{P}_{1}}\right)\left(1-\frac{\Theta\left(\mathrm{U}(\mathrm{t}), \mathrm{P}_{1}\right)}{\Theta(\mathrm{U}(\mathrm{t}), \mathrm{P}(\mathrm{t}))}\right) \leqslant 0
$$

Then we get that for all $\mathrm{U}(\mathrm{t}), \mathrm{L}(\mathrm{t}), \mathrm{I}(\mathrm{t}), \mathrm{O}(\mathrm{t}), \mathrm{P}(\mathrm{t}), \mathrm{C}(\mathrm{t})>0$ we have $\frac{d Z_{1}}{d t} \leqslant 0$, moreover $\frac{d Z_{1}}{d t}=0$ if and only if $\mathrm{U}(\mathrm{t})=\mathrm{U}_{1}, \mathrm{~L}(\mathrm{t})=\mathrm{L}_{1}, \mathrm{I}(\mathrm{t})=\mathrm{I}_{1}, \mathrm{O}(\mathrm{t})=\mathrm{O}_{1}, \mathrm{P}(\mathrm{t})=\mathrm{P}_{1}$ and $\mathrm{C}(\mathrm{t})=\mathrm{C}_{1}$. Applying L.I.P, we obtain that if $R_{0}^{G}>1$, then $E P_{1}^{G}$ is G.A.S.

\section{Numerical simulations}

In this section we perform numerical simulation for systems (2.1)-(2.6) and (3.1)-(3.6). We let $\tau=\tau_{i}$, $i=1,2, \ldots, 5$. In addition, we fix the values of parameters $\rho=10, \gamma=\mu=0.01, \zeta=\beta=0.3, v=\varepsilon=0.2$, $M_{2}=5, \xi=6, \wedge=0.1, \rho=0.4, \omega_{k}=0.001, \theta_{i}=0.6, i=1,2, \ldots, 5$ and $k=1,2,3$ and the remaining parameters will be changed.

4.1. Numerical simulations for model (2.1)-(2.6)

In this subsection we conduct numerical simulations for model (2.1)-(2.6).

\subsubsection{Stability of equilibria for different values of $M_{1}$}

We choose three different initial conditions as:

$\mathrm{IC} 1: \mathrm{U}(\mathrm{s})=800, \mathrm{~L}(\mathrm{~s})=2, \mathrm{I}(\mathrm{s})=4, \mathrm{O}(\mathrm{s})=7, \mathrm{P}(\mathrm{s})=1, \mathrm{C}(\mathrm{s})=8$; 
IC2: $\mathrm{U}(\mathrm{s})=600, \mathrm{~L}(\mathrm{~s})=3, \mathrm{I}(\mathrm{s})=7, \mathrm{O}(\mathrm{s})=12, \mathrm{P}(\mathrm{s})=2, \mathrm{C}(\mathrm{s})=13$;

IC3: $\mathrm{U}(\mathrm{s})=400, \mathrm{~L}(\mathrm{~s})=4, \mathrm{I}(\mathrm{s})=10, \mathrm{O}(\mathrm{s})=15, \mathrm{P}(\mathrm{s})=3, \mathrm{C}(\mathrm{s})=18, \mathrm{~s} \in[-\tau, 0]$.

We take $\tau=\vartheta=0.01$. Moreover, we consider two values of the parameter $M_{1}$ as:

(i) $M_{1}=0.5$, then we compute $R_{0}=0.9387<1$. Figure 1 shows that, for all IC1-IC3, the solution of the model tends to $\mathrm{EP}_{0}=(1000,0,0,0,0,0)$. It means that, $E P_{0}$ is G.A.S .

(ii) $M_{1}=10$, then we compute $R_{0}=3.1289>1$. Figure 1 shows that the solutions of the model converge to the equilibrium $\mathrm{EP}_{1}=(609.7,2.587,6.037,12.94,2.137,13.62)$ for all IC1-IC3. Then, $\mathrm{EP}_{1}$ is G.A.S.

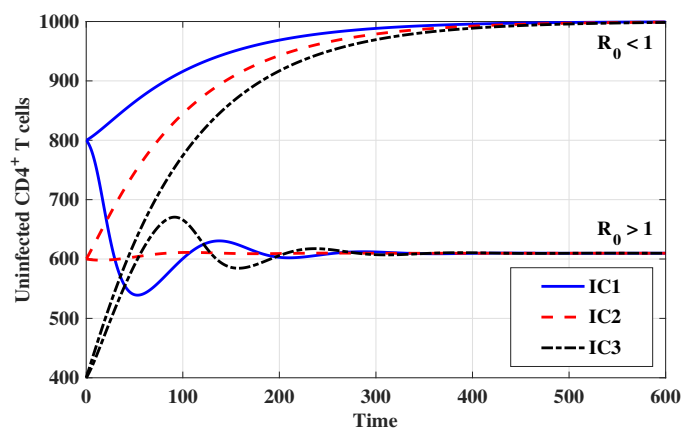

(a) The behavior of uninfected $\mathrm{CD} 4^{+} \mathrm{T}$ cells.

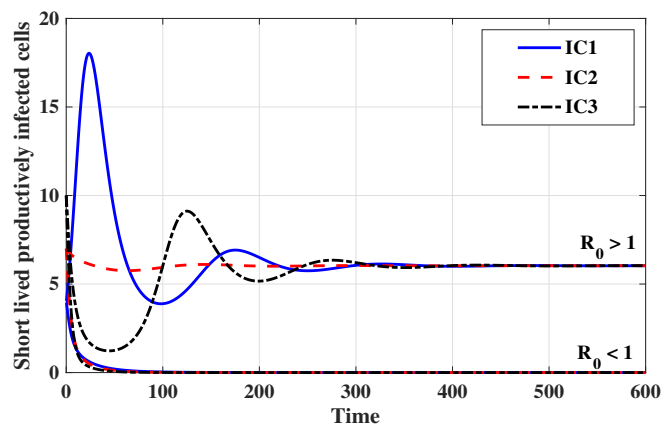

(c) The behavior of short lived productively infected (d) The behavior of long lived productively infected cells.

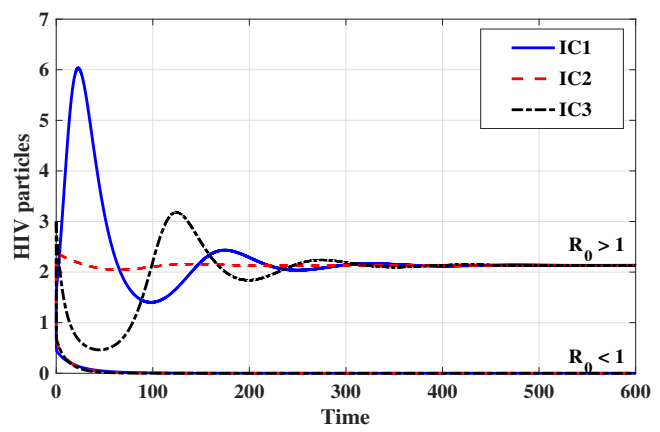

(e) The behavior of HIV particles.

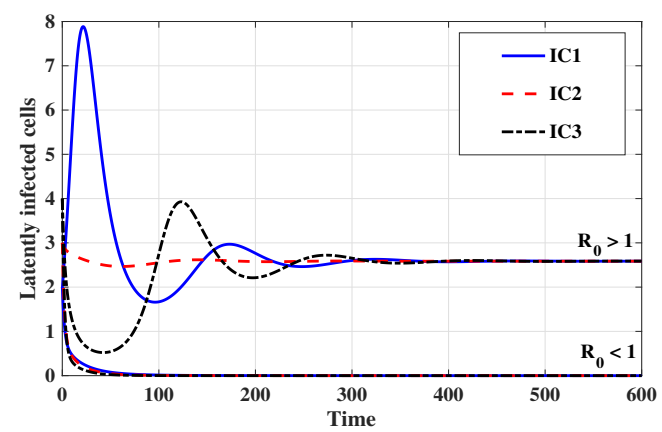

(b) The behavior of latently infected cells.

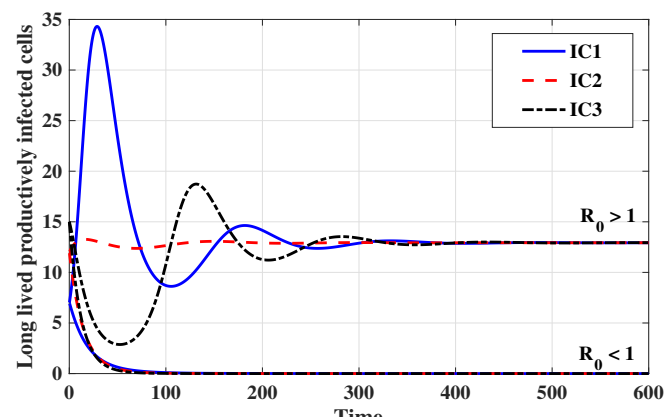
cells.

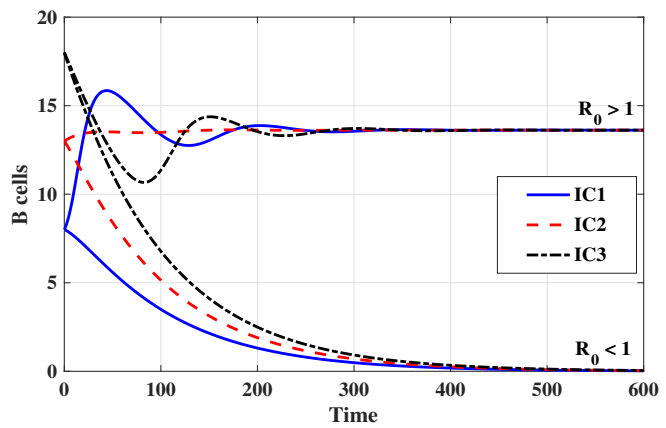

(f) The behavior of B cells.

Figure 1: Solution trajectories of model (2.1)-(2.6) for different values of $M_{1}$.

\subsubsection{The effect of $\tau$ on stability of equilibria}

In this case, we take $\vartheta=0.01, M_{1}=10$ and $\tau$ is varied. Moreover, we consider the following initial condition $\mathrm{U}(\mathrm{s})=600, \mathrm{~L}(\mathrm{~s})=1, \mathrm{I}(\mathrm{s})=2, \mathrm{O}(\mathrm{s})=4, \mathrm{P}(\mathrm{s})=1, \mathrm{C}(\mathrm{s})=8, \mathrm{~s} \in[-\tau, 0]$. Figure 2 shows that as $\tau$ 
is increased, the concentrations of latently infected cells, short lived productively infected cells, long lived productively infected cells, HIV particles and B cells are decreased, while the concentration of uninfected $\mathrm{CD}^{+} \mathrm{T}$ cells is increased until they reach the equilibrium point $\mathrm{EP}_{0}$. Moreover, we have the following observations:

(i) $E P_{1}$ is G.A.S when $0<\tau<0.9606$;

(ii) $E P_{0}$ is G.A.S when $\tau>0.9606$.

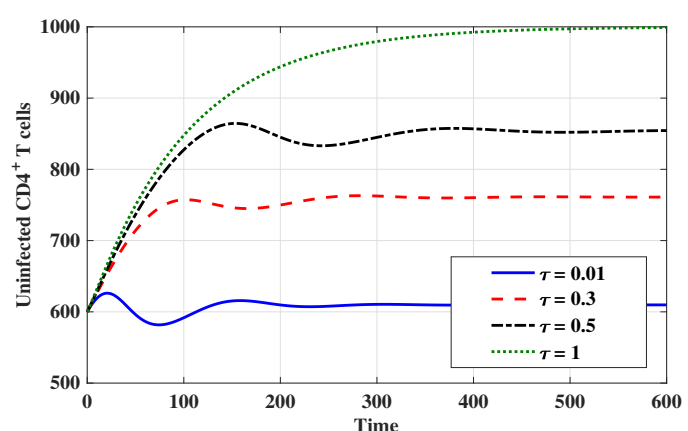

(a) The behavior of uninfected $\mathrm{CD} 4^{+} \mathrm{T}$ cells.

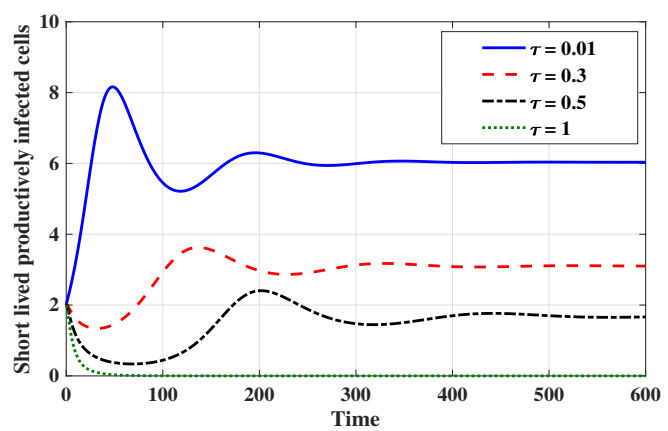

(c) The behavior of short lived productively infected (d) The behavior of long lived productively infected cells.

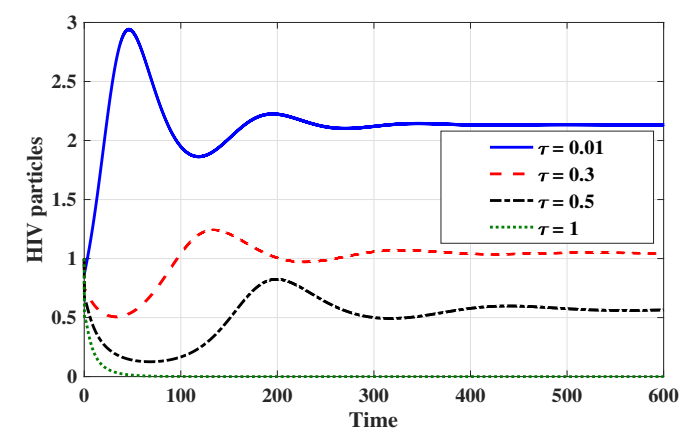

(e) The behavior of HIV particles.

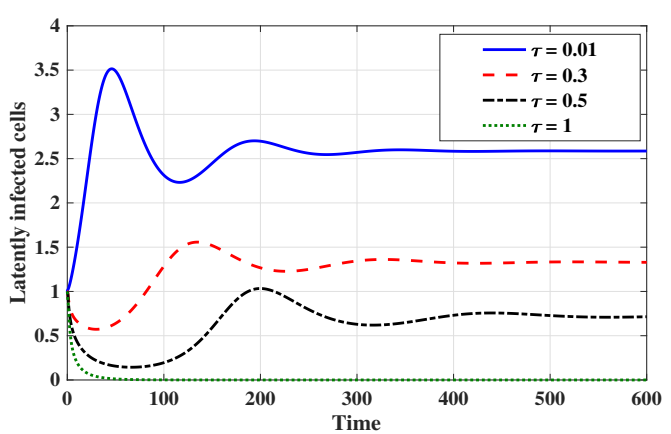

(b) The behavior of latently infected cells.

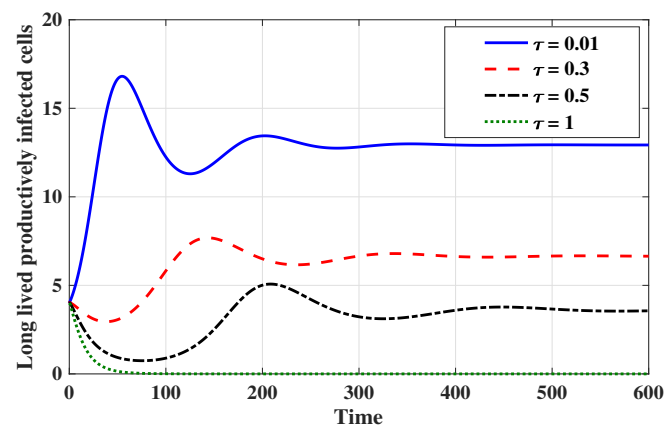
cells.

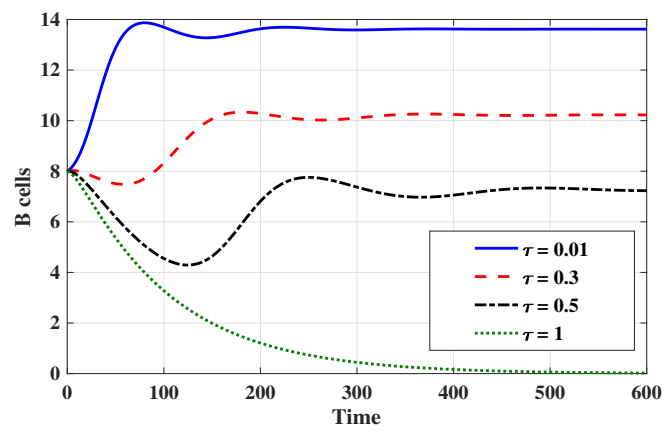

(f) The behavior of B cells.

Figure 2: Solution trajectories of model (2.1)-(2.6) for different values of $\tau$.

\subsubsection{Effect of B cell impairment parameter $\vartheta$ on the HIV dynamics.}

In this case, we take $\tau=0.01, M_{1}=10$ and $\vartheta$ is varied. Moreover, we consider the following initial condition $\mathrm{U}(\mathrm{s})=550, \mathrm{~L}(\mathrm{~s})=1, \mathrm{I}(\mathrm{s})=2, \mathrm{O}(\mathrm{s})=1, \mathrm{P}(\mathrm{s})=1, \mathrm{C}(\mathrm{s})=16, \mathrm{~s} \in[-\tau, 0]$. Figure 3 shows that as $\vartheta$ is increased, the concentrations of latently infected cells, short lived productively infected cells, long 
lived productively infected cells and HIV particles are increased, while the concentrations of uninfected $\mathrm{CD}^{+} \mathrm{T}$ cells and $\mathrm{B}$ cells are decreased. We note that the parameter $\vartheta$ has no effect on the stability of equilibria.

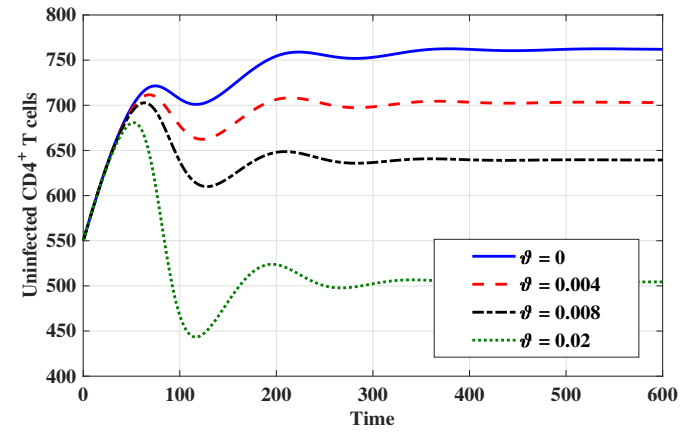

(a) The behavior of uninfected $\mathrm{CD}^{+}{ }^{+} \mathrm{T}$ cells.

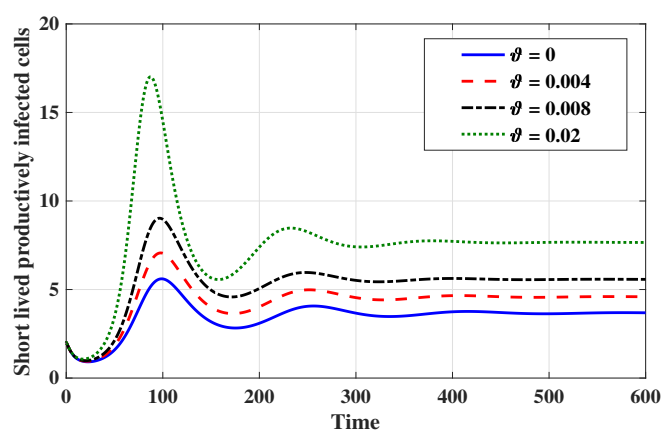

(c) The behavior of short lived productively infected cells.

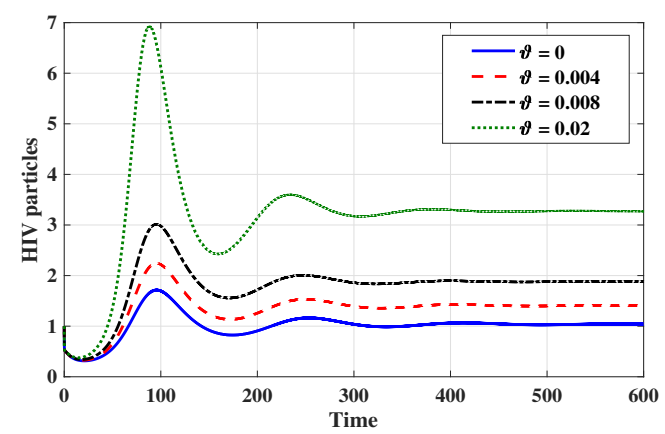

(e) The behavior of HIV particles.

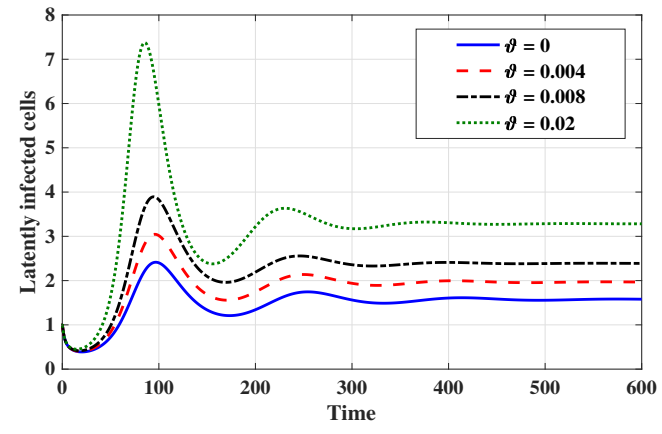

(b) The behavior of latently infected cells.

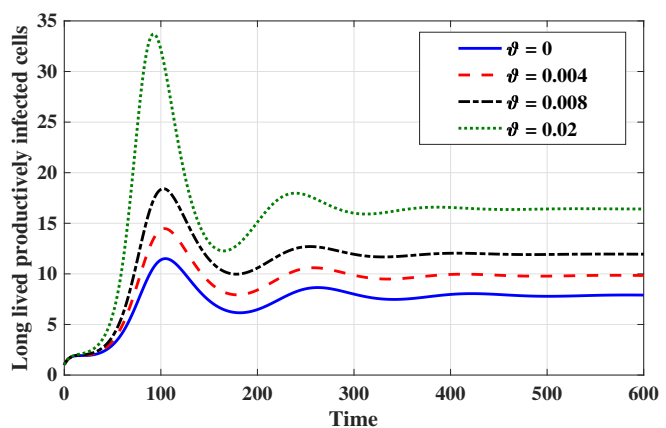

(d) The behavior of long lived productively infected cells.

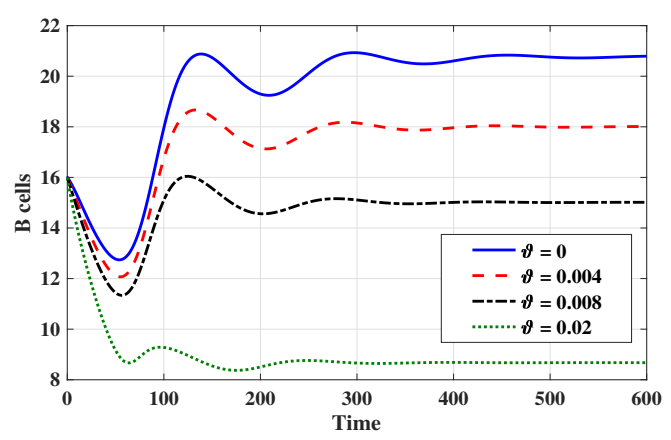

(f) The behavior of B cells.

Figure 3: Solution trajectories of model (2.1)-(2.6) for different values of $\vartheta$.

\subsection{Numerical simulations for model (3.1)-(3.6)}

We conduct numerical simulations for model (3.1)-(3.6) with specific incidence rate function

$$
\Theta(U, P)=\frac{U P}{1+\alpha_{1} P+\alpha_{2} U^{\prime}}
$$

where $\alpha_{1}$ and $\alpha_{2}$ are nonnegative parameters. We note that if $\alpha_{1}=\alpha_{2}=0$, then we obtain bilinear incidence which are given in model (2.1)-(2.6), if $\alpha_{1} \neq 0$ and $\alpha_{2}=0$, then we get saturated incidence, and if $\alpha_{1}=0$ and $\alpha_{2} \neq 0$, then we obtain Holling type-II incidence. We can easily see that $\Theta(U, P)$ is 
continuously differentiable function. Moreover, $\Theta(U, P)$ satisfies $\Theta(U, P)>0$, and $\Theta(0, P)=\Theta(U, 0)=0$ for all $U(t)>0$ and $P(t)>0$. Thus (A1) is satisfied. We have

$$
\frac{\partial \Theta(U, P)}{\partial U}=\frac{P+\alpha_{1} P^{2}}{\left(1+\alpha_{1} P+\alpha_{2} U\right)^{2}}>0, \quad \frac{\partial \Theta(U, P)}{\partial P}=\frac{U+\alpha_{2} U^{2}}{\left(1+\alpha_{1} P+\alpha_{2} U\right)^{2}}>0,
$$

for all $U(t)>0$ and $P(t)>0$. Moreover, $\frac{\partial \Theta(U, 0)}{\partial P}=\frac{U}{1+\alpha_{2} U}>0$ for all $U(t)>0$, then (A2) is satisfied.

We also have

$$
\frac{d}{d U}\left(\frac{\partial \Theta(U, 0)}{\partial P}\right)=\frac{1}{\left(1+\alpha_{2} U\right)^{2}}>0, \text { for all } U(t) \geqslant 0 \text {. }
$$

Then (A3) is satisfied. Finally we have

$$
\frac{\partial}{\partial P}\left(\frac{\Theta(U, P)}{P}\right)=\frac{-\alpha_{1} U}{\left(1+\alpha_{1} P+\alpha_{2} U\right)^{2}}<0, \quad \text { for all } U(t), P(t)>0 .
$$

Then (A4) is also satisfied. The basic reproduction number is given by

$$
R_{0}=\frac{\omega_{1} v M_{1} e^{-\theta_{1} \tau_{1}-\theta_{4} \tau_{4}}+(\zeta+v)\left[\omega_{2} M_{1} e^{-\theta_{2} \tau_{2}-\theta_{4} \tau_{4}}+\omega_{3} M_{2} e^{-\theta_{3} \tau_{3}-\theta_{5} \tau_{5}}\right]}{\xi(\zeta+v)\left(1+\alpha_{2} U_{0}\right)} U_{0} .
$$

In this subsection, we fix $\vartheta=\tau=0.01, M_{1}=10$ and choose $\alpha_{1}$ and $\alpha_{2}$.

\subsubsection{Effect of the saturation parameter $\alpha_{1}$ on the HIV dynamics}

In this case, we take $\alpha_{2}=0$ then $\Theta(U, P)$ represents the saturation incidence. We choose the following condition: $\mathrm{U}(\mathrm{s})=700, \mathrm{~L}(\mathrm{~s})=1.5, \mathrm{I}(\mathrm{s})=2, \mathrm{O}(\mathrm{s})=4, \mathrm{P}(\mathrm{s})=1, \mathrm{C}(\mathrm{s})=10, \mathrm{~s} \in[-\tau, 0]$. Figure 4 shows that as $\alpha_{1}$ is increased, the concentration of the uninfected $\mathrm{CD}^{+}{ }^{+} \mathrm{T}$ cells is increased, while the concentrations of latently infected cells, short lived productively infected cells, long lived productively infected cells, HIV particles, and B cells are decreased. We note that the parameter $\alpha_{1}$ has no effect on the stability of equilibria. The reason is that $R_{0}$ does not depend on the parameter $\alpha_{1}$.

\subsubsection{Effect of Holling type-II constant $\alpha_{2}$ on HIV dynamics}

For this case, we take $\alpha_{1}=0$ then $\Theta(\mathrm{U}, \mathrm{P})$ represents the Holling type-II incidence. Let us choose the following condition: $\mathrm{U}(\mathrm{s})=700, \mathrm{~L}(\mathrm{~s})=2, \mathrm{I}(\mathrm{s})=2, \mathrm{O}(\mathrm{s})=4, \mathrm{P}(\mathrm{s})=1, \mathrm{C}(\mathrm{s})=10, \mathrm{~s} \in[-\tau, 0]$. We suggest different values of $\alpha_{2}$ to see its effect on the solution of the model as we can see in Figure 5. Moreover, we conclude the following results:

(i) $E P_{1}^{G}$ is G.A.S, when $0 \leqslant \alpha_{2}<0.0031$;

(ii) $E P_{0}^{G}$ is G.A.S, when $\alpha_{2}>0.0031$.

This means that $\alpha_{2}$ can play the role of controller which can be designed to stabilize the system around the infection-free equilibrium $\mathrm{EP}_{0}^{\mathrm{G}}$.

\subsubsection{Effect of antiviral treatment on the HIV dynamics}

Let us introduce the HIV dynamics model under the effect of highly active antiretroviral therapies (HAART) as:

$$
\begin{aligned}
& \dot{\mathrm{U}}(\mathrm{t})=\rho-\gamma \mathrm{U}(\mathrm{t})-\left(1-\epsilon_{\mathrm{r}}\right)\left(\omega_{1}+\omega_{2}+\omega_{3}\right) \frac{\mathrm{U}(\mathrm{t}) \mathrm{P}(\mathrm{t})}{1+\alpha_{2} \mathrm{U}(\mathrm{t})}, \\
& \dot{\mathrm{L}}(\mathrm{t})=\left(1-\epsilon_{\mathrm{r}}\right) \omega_{1} e^{-\theta_{1} \tau_{1}} \frac{\mathrm{U}\left(\mathrm{t}-\tau_{1}\right) \mathrm{P}\left(\mathrm{t}-\tau_{1}\right)}{1+\alpha_{2} \mathrm{U}\left(\mathrm{t}-\tau_{1}\right)}-(\zeta+v) \mathrm{L}(\mathrm{t}), \\
& \dot{\mathrm{I}}(\mathrm{t})=\left(1-\epsilon_{\mathrm{r}}\right) \omega_{2} e^{-\theta_{2} \tau_{2}} \frac{\mathrm{U}\left(\mathrm{t}-\tau_{2}\right) \mathrm{P}\left(\mathrm{t}-\tau_{2}\right)}{1+\alpha_{2} \mathrm{U}\left(\mathrm{t}-\tau_{2}\right)}+v \mathrm{~L}(\mathrm{t})-\beta \mathrm{I}(\mathrm{t}),
\end{aligned}
$$




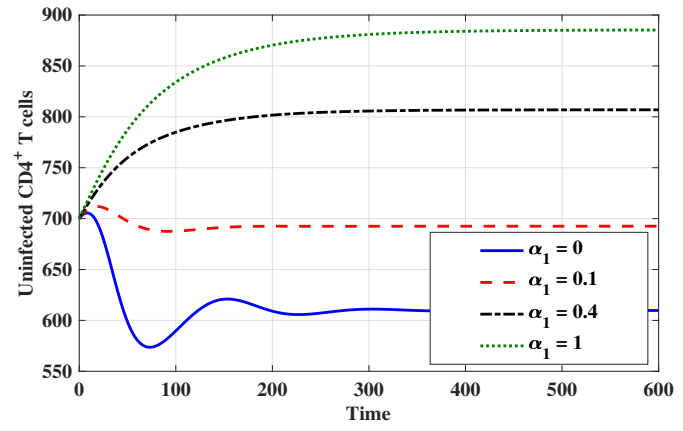

(a) The behavior of uninfected $\mathrm{CD} 4^{+} \mathrm{T}$ cells.

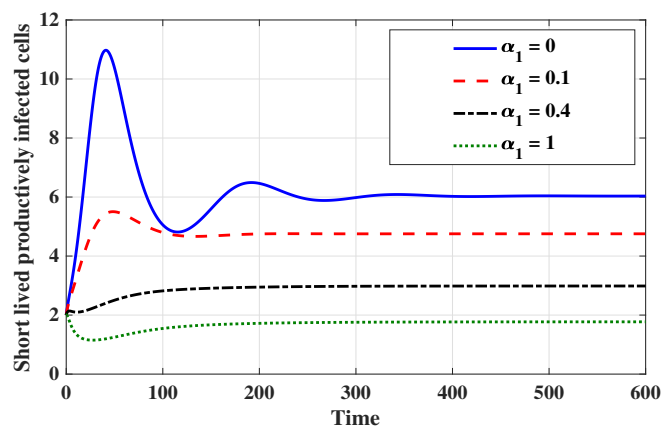

(c) The behavior of short lived productively infected (d) cells.

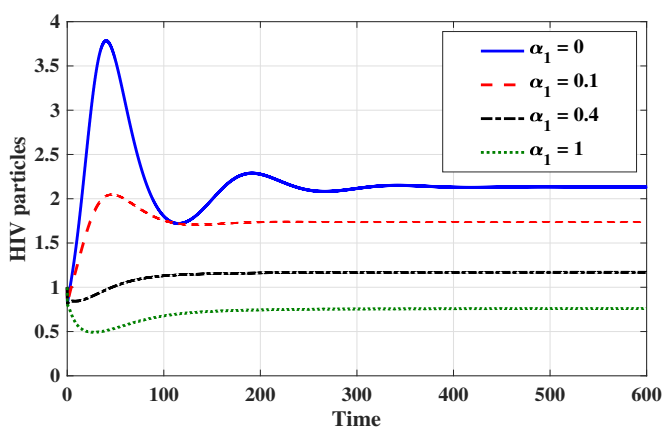

(e) The behavior of HIV particles.

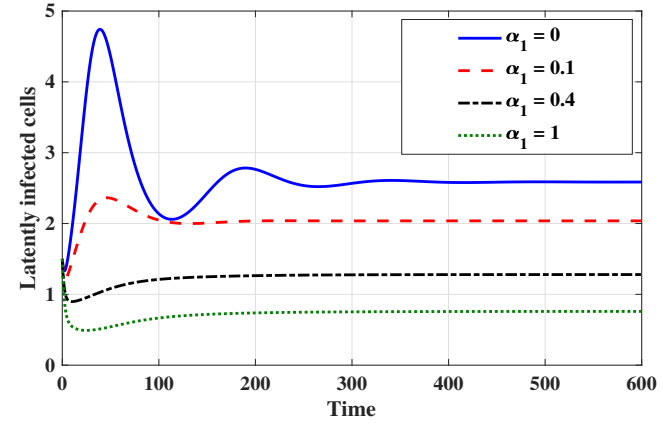

(b) The behavior of latently infected cells.

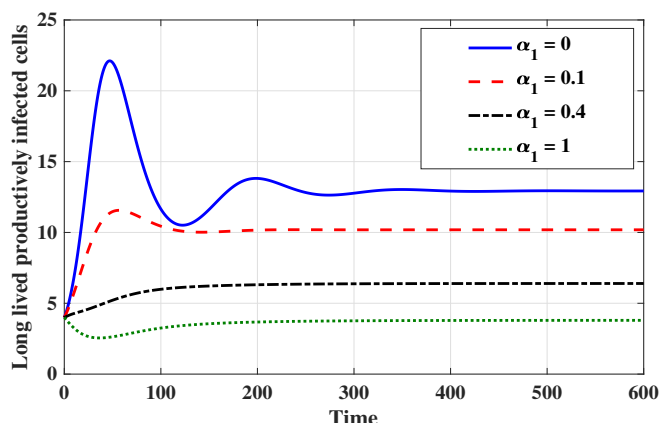
cells.

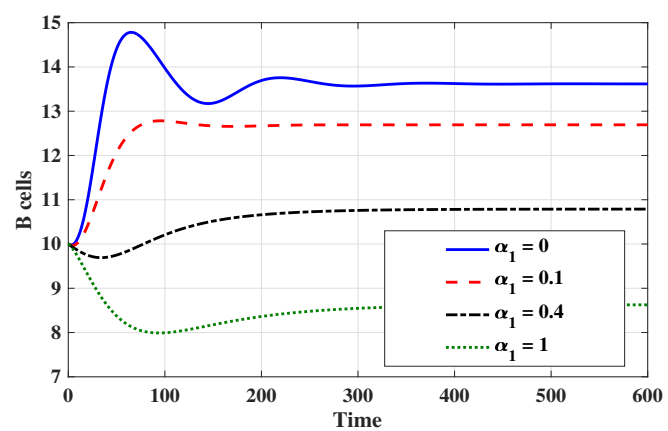

(f) The behavior of B cells.

Figure 4: Solution trajectories of model (3.1)-(3.6) for different values of $\alpha_{1}$.

$$
\begin{aligned}
& \dot{\mathrm{O}}(\mathrm{t})=\left(1-\epsilon_{\mathrm{r}}\right) \omega_{3} e^{-\theta_{3} \tau_{3}} \frac{\mathrm{U}\left(\mathrm{t}-\tau_{3}\right) \mathrm{P}\left(\mathrm{t}-\tau_{3}\right)}{1+\alpha_{2} \mathrm{U}\left(\mathrm{t}-\tau_{3}\right)}-\Lambda \mathrm{O}(\mathrm{t}), \\
& \dot{\mathrm{P}}(\mathrm{t})=\left(1-\epsilon_{\mathrm{p}}\right) \beta M_{1} e^{-\theta_{4} \tau_{4}} \mathrm{I}\left(\mathrm{t}-\tau_{4}\right)+\left(1-\epsilon_{\mathrm{p}}\right) \wedge M_{2} e^{-\theta_{5} \tau_{5}} \mathrm{O}\left(\mathrm{t}-\tau_{5}\right)-\xi \mathrm{P}(\mathrm{t})-\rho \mathrm{P}(\mathrm{t}) \mathrm{C}(\mathrm{t}), \\
& \dot{\mathrm{C}}(\mathrm{t})=\varepsilon \mathrm{P}(\mathrm{t})-\mu \mathrm{C}(\mathrm{t})-\vartheta \mathrm{P}(\mathrm{t}) \mathrm{C}(\mathrm{t}),
\end{aligned}
$$

where $\epsilon_{\mathrm{r}} \in[0,1]$ is the efficacy of the reverse transcriptase inhibitor drug, while $\epsilon_{\mathrm{p}} \in[0,1]$ is the efficacy of the protease inhibitor drug. If $\epsilon_{r}=\epsilon_{p}=0$, then the HAART has no effect, if $\epsilon_{r}=\epsilon_{p}=1$, the HIV growth is completely stopped. Let $\epsilon=\epsilon_{r}=\epsilon_{p}$, consequently, the parameter $R_{0}(\epsilon)$ is given by

$$
R_{0}(\epsilon)=\frac{(1-\epsilon)^{2}\left[\omega_{1} v M_{1} e^{-\theta_{1} \tau_{1}-\theta_{4} \tau_{4}}+(\zeta+v)\left(\omega_{2} M_{1} e^{-\theta_{2} \tau_{2}-\theta_{4} \tau_{4}}+\omega_{3} M_{2} e^{-\theta_{3} \tau_{3}-\theta_{5} \tau_{5}}\right)\right]}{\xi(\zeta+v)\left(1+\alpha_{2} U_{0}\right)} U_{0}
$$




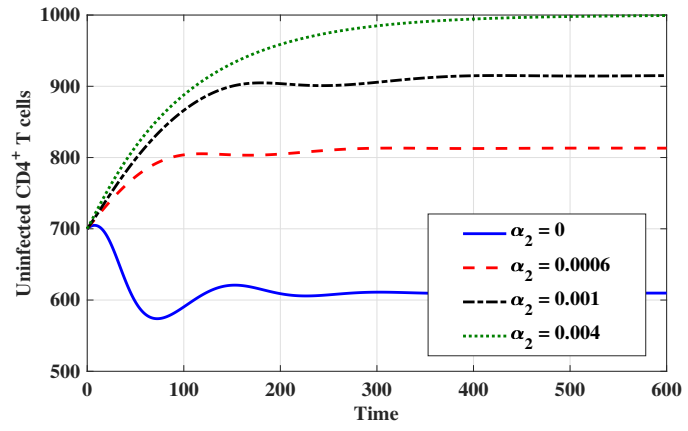

(a) The behavior of uninfected $\mathrm{CD}^{+} \mathrm{T}$ cells.

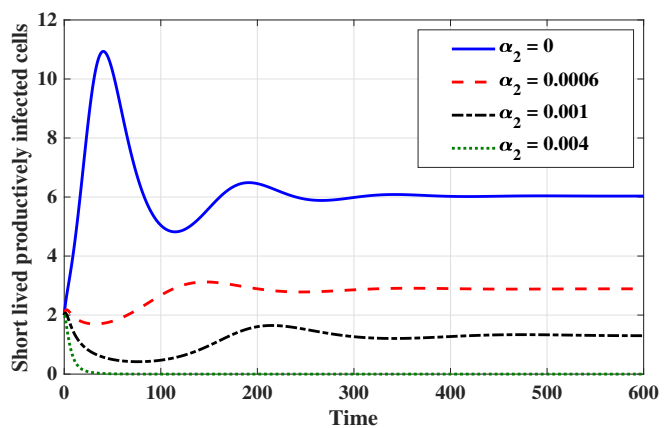

(c) The behavior of short lived productively infected (d) cells.

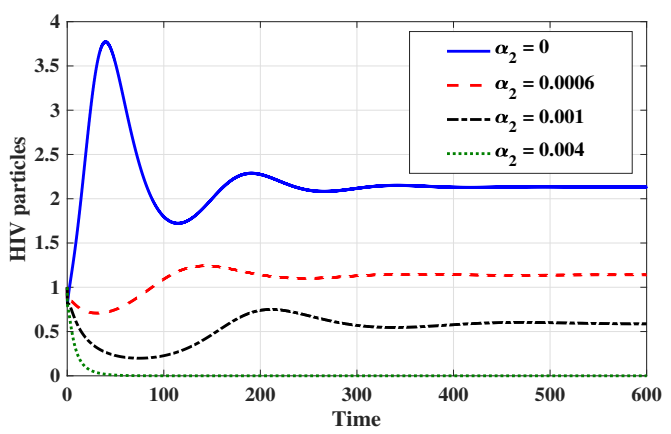

(e) The behavior of HIV particles.

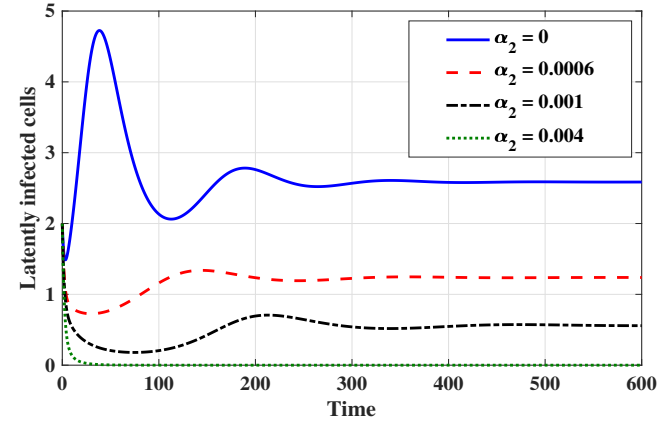

(b) The behavior of latently infected cells.

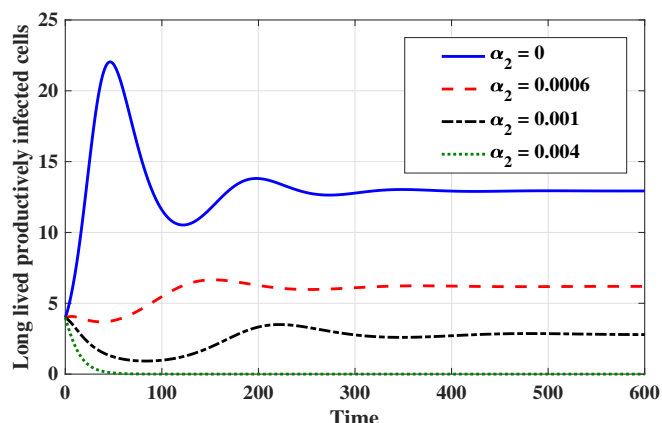
cells.

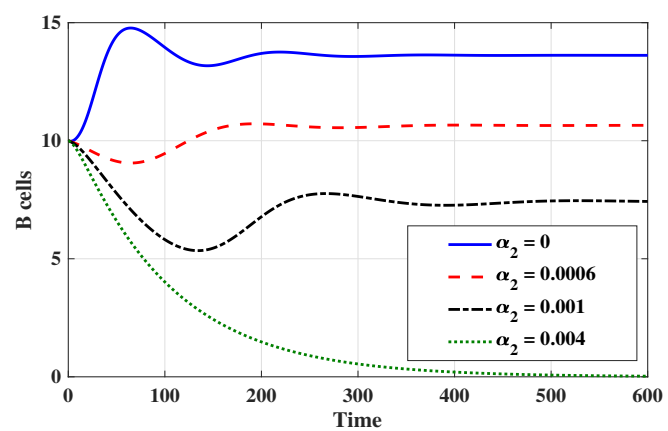

(f) The behavior of B cells.

Figure 5: Solution trajectories of model (3.1)-(3.6) for different values of $\alpha_{2}$.

Since the goal is to clear the HIV from the body, then we have to determine the drug efficacies that make $\mathrm{R}_{0}(\epsilon)<1$ for model (4.1)-(4.6). Then

$$
(1-\epsilon)^{2}<\frac{1}{R_{0}(0)}
$$

Since, $0 \leqslant \epsilon \leqslant 1$, then for $\epsilon^{\min }<\epsilon \leqslant 1$, $\operatorname{EP}_{0}^{G}$ is G.A.S, where $\epsilon^{\min }=\max \left\{0,1-\frac{1}{\sqrt{R_{0}(0)}}\right\}$. We take $\alpha_{2}=0.0006$, then, we find the following:

(i) if $0.2849<\epsilon \leqslant 1$, then $R_{0}(\epsilon)<1$ and $E P_{0}^{G}$ is G.A.S;

(ii) if $0 \leqslant \epsilon<0.2849$, then $R_{0}(\epsilon)>1$ and $E P_{1}^{G}$ is G.A.S.

We consider the following initial condition $\mathrm{U}(0)=850, \mathrm{~L}(0)=0.7, \mathrm{I}(0)=2, \mathrm{O}(0)=4, \mathrm{P}(0)=0.6, \mathrm{C}(0)=8$ to show in Figure 6 the solution trajectories of model (4.1)-(4.6) for different values of $\epsilon$. Clearly from 
the figure we can see that, the increasing of $\epsilon$ will increase the concentration of the uninfected CD4 ${ }^{+} \mathrm{T}$ cells and decrease the concentrations of latently infected cells, short lived productively infected cells, long lived productively infected cells, HIV particles, and B cells.

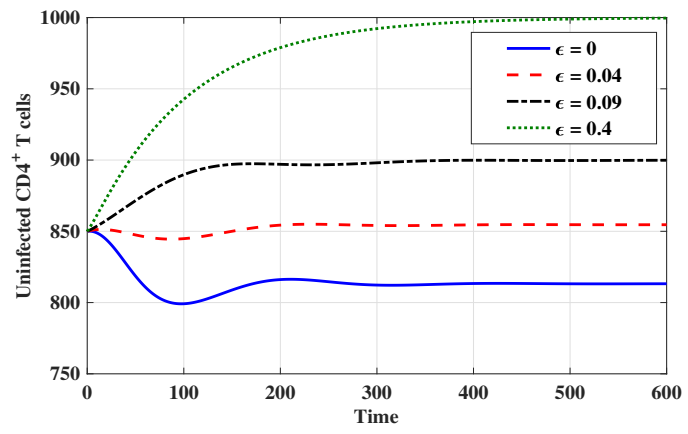

(a) The behavior of uninfected $\mathrm{CD} 4^{+} \mathrm{T}$ cells.

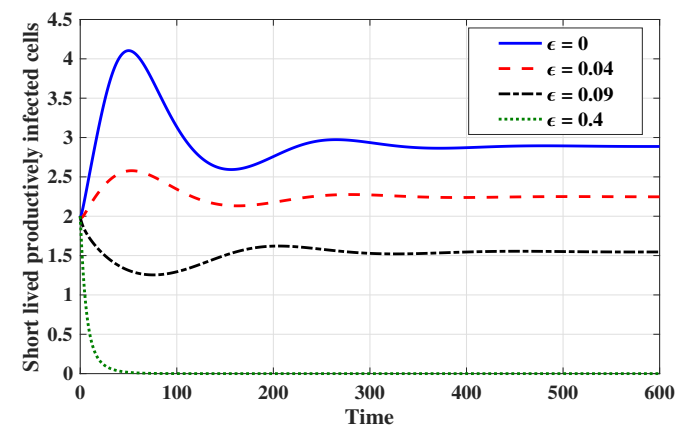

(c) The behavior of short lived productively infected cells.

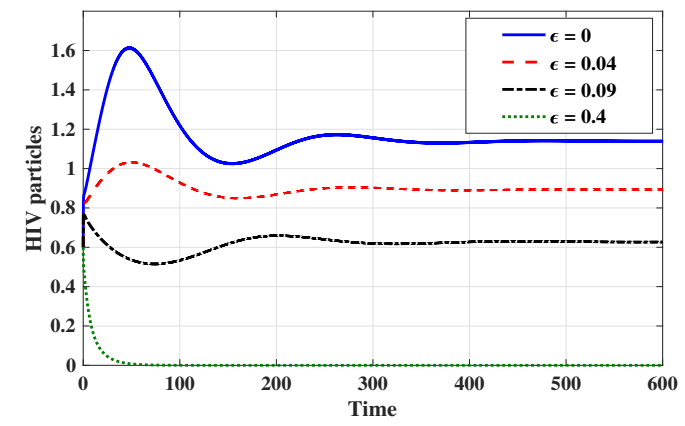

(e) The behavior of HIV particles.

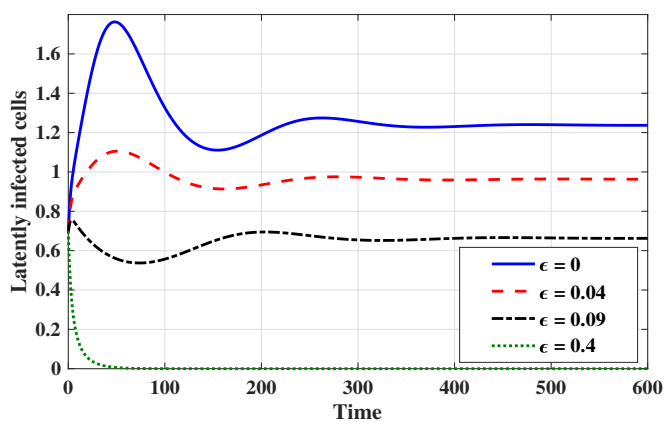

(b) The behavior of latently infected cells.

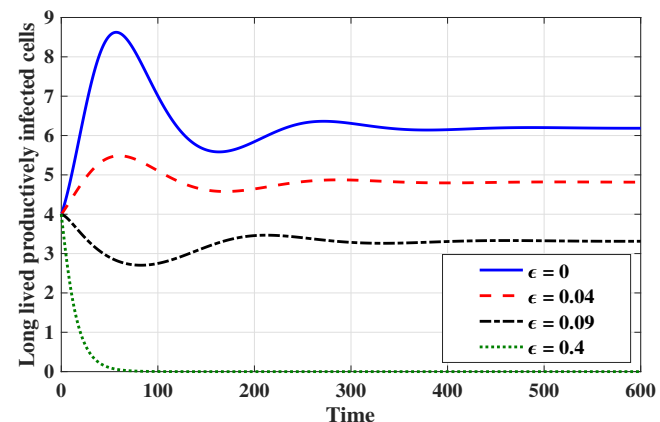

(d) The behavior of long lived productively infected cells.

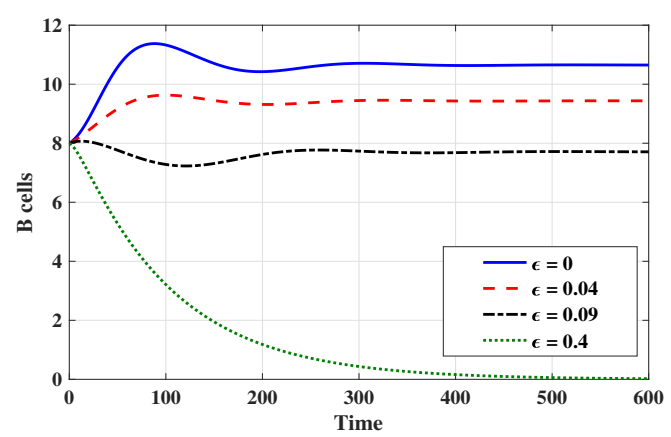

(f) The behavior of B cells.

Figure 6: Solution trajectories of model (4.1)-(4.6) for different values of $\epsilon$.

\section{Conclusion}

In the literature, various mathematical models of virus dynamics have investigated the impairment of CTL functions. However, the dysfunction of B cell could happened during the HIV infection as it has been reported in several papers. In this work, we have studied HIV infection models with five delays including the impairment of B cell functions. We have taken into account three types of infected cells: short-lived productively infected cells (these cells live for short time and produce large amount of HIV particles), long-lived productively infected cells (which live for long time and produce small amount of 
HIV particles) and latently infected cells (such cells contain the HIV but are not producing it). Bilinear and general incidence rates have been considered in the first and second model, respectively. We have shown that, the solutions of the models are non-negative and ultimately bounded which ensure the wellposedness of the models. We have derived the basic reproduction number $R_{0}$ which fully determines the existence and stability of the two equilibria of the models. We have examined the global stability of the two equilibria of the models by using Lyapunov method and LaSalle's invariance principle. We have proven that (i) if $R_{0}<1$, then the infection-free equilibrium is G.A.S and the HIV is predicted to be completely cleared from the HIV infected individuals, (ii) if $R_{0}>1$, then the endemic equilibrium is G.A.S and a chronic HIV infection is attained. We have conducted numerical simulations and have shown that both the theoretical and numerical results are consistent. The results show that, when the B cells loss their functions during the HIV infection, the number of antibodies produced from the B cells are decreased and then the number of HIV particles are increased. Therefore, HAART is needed to improve the health of the HIV infected patient.

\section{References}

[1] S. Amu, N. Ruffin, B. Rethi, F. Chiodi, Impairment of B-cell functions during HIV-1 infection, AIDS, 27 (2013), 23232334. 1

[2] N. Bellomo, Y. Tao, Stabilization in a chemotaxis model for virus infection, Discrete. Cont. Dyn Syst.-S, 13 (2020), 105-117. 1

[3] J. N. Blankson, D. Persaud, R. F. Siliciano, The challenge of viral reservoirs in HIV-1 infection, Ann. Rev. Med., 53 (2002), 557-593.

[4] F. Chiodi, G. Scarlatti, HIV-Induced damage of B cells and production of HIV neutralizing antibodies, Front. Immunol., 9 (2018), 4 pages. 1

[5] R. V. Culshaw, S. Ruan, A delay-differential equation model of HIV infection of CD4 ${ }^{+}$T-cells, Math. Biosci., 165 (2000), 27-39.

[6] R. V. Culshaw, S. Ruan, G. Webb, A mathematical model of cell-to-cell spread of HIV-1 that includes a time delay, J. Math. Biol., 46 (2003), 425-444. 1

[7] A. De Milito, B lymphocyte dysfunctions in HIV Infection, Curr. HIV. Res., 2 (2004), 11-21. 1

[8] A. M. Elaiw, Global properties of a class of HIV models, Nonlinear Anal. Real World Appl., 11 (2010), 2253-2263. 1

[9] A. M. Elaiw, Global dynamics of an HIV infection model with two classes of target cells and distributed delays, Discrete Dyn. Nat. Soc., 2012 (2012), 13 pages.

[10] A. M. Elaiw, Global properties of a class of virus infection models with multitarget cells, Nonlinear Dynam., 69 (2012), 423-435.

[11] A. M. Elaiw, R. M. Abukwaik, E. O. Alzahrani, Global properties of a cell mediated immunity in HIV infection model with two classes of target cells and distributed delays, Int. J. Biomath., 7 (2014), 25 pages.

[12] A. M. Elaiw, A. A. Almatrafi, A. D. Hobiny, K. Hattaf, Global properties of a general latent pathogen dynamics model with delayed pathogenic and cellular infections, Discrete Dyn. Nat. Soc., 2019 (2019), 18 pages.

[13] A. M. Elaiw, N. A. Almuallem, Global properties of delayed-HIV dynamics models with differential drug efficacy in cocirculating target cells, Appl. Math. Comput., 265 (2015), 1067-1089. 1

[14] A. M. Elaiw, N. A. Almuallem, Global dynamics of delay-distributed HIV infection models with differential drug efficacy in cocirculating target cells, Math. Methods Appl. Sci., 39 (2016), 4-31. 1

[15] A. M. Elaiw, M. A. Alshaikh, Stability analysis of a general discrete-time pathogen infection model with humoral immunity, J. Differ. Equ. Appl., 2019 (2019), 24 pages.

[16] A. M. Elaiw, M. A. Alshaikh, Stability of discrete-time HIV dynamics models with three categories of infected CD4 ${ }^{+}$ T-cells, Adv. Difference Equ., 2019 (2019), 24 pages.

[17] A. M. Elaiw, N. H. AlShamrani, Global properties of nonlinear humoral immunity viral infection models, Int. J. Biomath., 8 (2015), 53 pages.

[18] A. M. Elaiw, N. H. AlShamrani, Global stability of humoral immunity virus dynamics models with nonlinear infection rate and removal, Nonlinear Anal. Real World Appl., 26 (2015), 161-190. 3

[19] A. M. Elaiw, N. H. AlShamrani, Stability of a general delay-distributed virus dynamics model with multi-staged infected progression and immune response, Math. Methods Appl. Sci., 40 (2017), 699-719. 1

[20] A. M. Elaiw, N. H. AlShamrani, Stability of an adaptive immunity pathogen dynamics model with latency and multiple delays, Math. Methods Appl. Sci., 36 (2018), 125-142.

[21] A. M. Elaiw, N. H. AlShamrani, Stability of a general adaptive immunity virus dynamics model with multi-stages of infected cells and two routes of infection, Math. Methods Appl. Sci., 2019 (2019), 15 pages.

[22] A. M. Elaiw, S. F. Alshehaiween, A. D. Hobiny, Global properties of delay-distributed HIV dynamics model including impairment of B-cell functions, Mathematics, 7 (2019), 27 pages. 
[23] A. M. Elaiw, S. A. Azoz, Global properties of a class of HIV infection models with Beddington-DeAngelis functional response, Math. Methods Appl. Sci., 36 (2013), 383-394.

[24] A. M. Elaiw, E. K. Elnahary, Analysis of general humoral immunity HIV dynamics model with HAART and distributed delays, Mathematics, 7 (2019), 35 pages.

[25] A. M. Elaiw, E. K. Elnahary, A. A. Raezah, Effect of cellular reservoirs and delays on the global dynamics of HIV, Adv. Difference Equ., 2018 (2018), 36 pages.

[26] A. M. Elaiw, I. A. Hassanien, S. A. Azoz, Global stability of HIV infection models with intracellular delays, J. Korean Math. Soc., 49 (2012), 779-794.

[27] A. M. Elaiw, A. G. Hobiny, A. D. Al Agha, Global dynamics of reaction-diffusion oncolytic M1 virotherapy with immune response, Appl. Math. Comput., 367 (2019), 124758.

[28] A. M. Elaiw, A. A. Raezah, Stability of general virus dynamics models with both cellular and viral infections and delays, Math. Methods Appl. Sci., 40 (2017), 5863-5880.

[29] A. M. Elaiw, A. A. Raezah, S. A. Azoz, Stability of delayed HIV dynamics models with two latent reservoirs and immune impairment, Adv. Difference Equ., 2018 (2018), 25 pages.

[30] L. Gibelli, A. Elaiw, M. A. Alghamdi, A. M. Althiabi, Heterogeneous population dynamics of active particles: Progression, mutations, and selection dynamics, Math. Models Methods Appl. Sci., 27 (2017), 617-640. 1

[31] J. K. Hale, S. M. Verduyn Lunel, Introduction to functional differential equations, Springer-Verlag, New York (1993). $2,2.1$

[32] A. D. Hobiny, A. M. Elaiw, A. A. Almatrafi, Stability of delayed pathogen dynamics models with latency and two routes of infection, Adv. Difference Equ., 2018 (2018), 26 pages. 1

[33] G. Huang, Y. Takeuchi, W. Ma, Lyapunov functionals for delay differential equations model of viral infections, SIAM J. Appl. Math., 70 (2010), 2693-2708. 3

[34] Y. Kuang, Delay Differential Equations with applications in population dynamics, Academic Press, San Diego, (1993). 2

[35] B. Li, Y. M. Chen, X. J. Lu, S. Q. Liu, A delayed HIV-1 model with virus waning term, Math. Biosci. Eng., 13 (2016), 135-157. 1

[36] D. Li, W. Ma, Asymptotic properties of a HIV-1 infection model with time delay, J. Math. Anal. Appl., 335 (2007), 683-691. 1

[37] J. Z. Lin, R. Xu, X. H. Tian, Threshold dynamics of an HIV-1 virus model with both virus-to-cell and cell-to-cell transmissions, intracellular delay, and humoral immunity, Appl. Math. Comput., 315 (2017), 516-530. 1

[38] H. J. Liu, J.-F. Zhang, Dynamics of two time delays differential equation model to HIV latent infection, Phys. A, 514 (2019), 384-395. 1

[39] Y. Lv, Z. X. Hu, F. C. Liao, The stability and Hopf bifurcation for an HIV model with saturated infection rate and double delays, Int. J. Biomath., 11 (2018), 43 pages. 1

[40] P. Lydyard, A. Whelan, M. Fanger, BIOS Instant notes in immunology, Taylor \& Francis, London, (2005). 1

[41] H. Miao, X. Abdurahman, Z. D. Teng, L. Zhang, Dynamical analysis of a delayed reaction-diffusion virus infection model with logistic growth and humoral immune impairment, Chaos Solitons Fractals, 110 (2018), 280-291. 1

[42] H. Miao, Z. D. Teng, C. J. Kang, A. Muhammadhaji, Stability analysis of a virus infection model with humoral immunity response and two time delays, Math. Methods Appl. Sci., 39 (2016), 3434-3449. 1

[43] A. Murase, T. Sasaki, T. Kajiwara, Stability analysis of pathogen-immune interaction dynamics, J. Math. Biol., 51 (2005), 247-267. 1

[44] P. W. Nelson, J. D. Murray, A. S. Perelson, A model of HIV-1 pathogenesis that includes an intracellular delay, Math. Biosci., 163 (2000), 201-215. 1

[45] M. A. Nowak, C. R. M. Bangham. Population dynamics of immune responses to persistent viruses, Science, 272 (1996), 74-79. 1

[46] M. A. Nowak, R. M. May, Virus dynamics: Mathematical Principles of Immunology and Virology, Oxford University Press, Oxford, (2000). 1

[47] A. S. Perelson, P. W. Nelson, Mathematical analysis of HIV-1 dynamics in vivo, SIAM. Rev., 41 (1999), 3-44. 1

[48] S. K. Sahani, Yashi, Effects of eclipse phase and delay on the dynamics of HIV infection, J. Biol. Systems, 26 (2018), 421-454. 1

[49] H. Y. Shu, L. Wang, J. Watmough, Global stability of a nonlinear viral infection model with infinitely distributed intracellular delays and CTL immune responses, SIAM J. Appl. Math., 73 (2013), 1280-1302. 3

[50] T. L. Wang, Z. X. Hu, F. C. Liao, Stability and Hopf bifurcation for a virus infection model with delayed humoral immunity response, J. Math. Anal. Appl., 411 (2014), 63-74. 1

[51] T. L. Wang, Z. X. Hu, F. C. Liao, W. B. Ma, Global stability analysis for delayed virus infection model with general incidence rate and humoral immunity, Math. Comput. Simulation, 89 (2013), 13-22. 1

[52] L. C. Wang, M. Y. Li, Mathematical analysis of the global dynamics of a model for HIV infection of CD4 ${ }^{+} \mathrm{T}$ cells, Math. Biosci., 200 (2006), 44-57. 1

[53] S. F. Wang, D. Y. Zou, Global stability of in-host viral models with humoral immunity and intracellular delays, Appl. Math. Model., 36 (2012), 1313-1322. 1

[54] Y. Zhao, D. T. Dimitrov, H. Liu, Y. Kuang, Mathematical insights in evaluating state dependent effectiveness of HIV prevention interventions, Bull. Math. Biol., 75 (2013), 649-675. 1 